\title{
Piezoelectric energy production
}

J. Nunes-Pereira ${ }^{1,2}$, P. Costa ${ }^{1,3}$, and S. Lanceros-Mendez ${ }^{4,5}$

${ }^{1}$ Centro/Departamento de Física, Universidade do Minho, Campus de Gualtar, 4710-057 Braga, Portugal

2 C-MAST - Centre for Mechanical and Aerospace Sciences and Technologies, Universidade da Beira Interior, 6200-001 Covilhã, Portugal

${ }^{3}$ Institute for Polymers and Composites IPC/I3N, University of Minho, 4800-058 Guimarães, Portugal

${ }^{4}$ BCMaterials, Parque Científico y Tecnológico de Bizkaia, 48160 Derio, Spain

${ }^{5}$ IKERBASQUE, Basque Foundation for Science, 48013 Bilbao, Spain

Contact:

J. Nunes-Pereira - jpereira@ fisica.uminho.pt; +351966141759

P. Costa - pcosta@ fisica.uminho.pt; +351936396162

S. Lanceros-Mendez - senentxu.lanceros@ @cmaterials.net; +34946128811

\begin{abstract}
The concept of piezoelectric energy production is based on energy harvesting devices using as generation materials single crystals, ceramics, polymers and composites. These production systems can harvest wasted environmental energy and convert it essentially into electrical energy. There are different nano- and micro-scale power harvesters which are increasingly useful for powering mobile electronics and low power devices, even in hardly accessible areas. Despite many efforts in the development of new materials, the most widely used materials in device applications remain the ceramics of the PZT family, since they still present the higher output performances in the range of $\mathrm{mW}$ of generated power.
\end{abstract}




\section{Introduction}

This chapter provides an introduction to energy harvesting based on piezoelectric materials. Piezoelectric materials are widely used as smart materials due to their wide bandwidth, fast electromechanical response, relatively low-power requirements and high generated forces. These materials can operate either as a motor or as a generator depending on the application. Thus, piezoelectric materials have been investigated in order to improve their maximum displacements with a minimal input voltage, for motor applications, and to produce the maximum output voltage with a minimal applied stress or strain, for generator applications. The later will be the main focus of the present chapter.

Piezoelectric generators work as a transducer that collets useful energy from wasted sources, such as mechanical vibrations, that can be used to power micro devices.

Piezoelectric materials integrated in microelectromechanical systems are commonly used actually in the automotive industry, smart phones and several low power applications. Further, with the rapid development of low power portable electronics and wearable sensors, energy harvesting is highlighted as a potential alternative to conventional batteries. Similarly, another emergent area for piezoelectric energy harvesting applications is the development of self-powered sensors in remote or difficult to reach locations, in order to avoid the used of batteries with a limited service-life.

\section{Energy Harvesting}

In the last years the term energy harvesting appears to describe a device or system that capture or harvest wasted or unused environmental energy and converts it essentially, but not exclusively, into electrical energy. It is a promising technology to provide solutions for the area of energy and its environmental impact, decreasing the energy waste. There are different nano- and micro-scale power harvesters such as thermoelectric, thermophotovoltaic, piezoelectric and microbial fuel cell, among others, which are increasingly useful for powering mobile electronics, even in remote areas, without the need for large power storage elements. Their positive prospects and benefits are summarized, for several technologies, in Figure 1. 


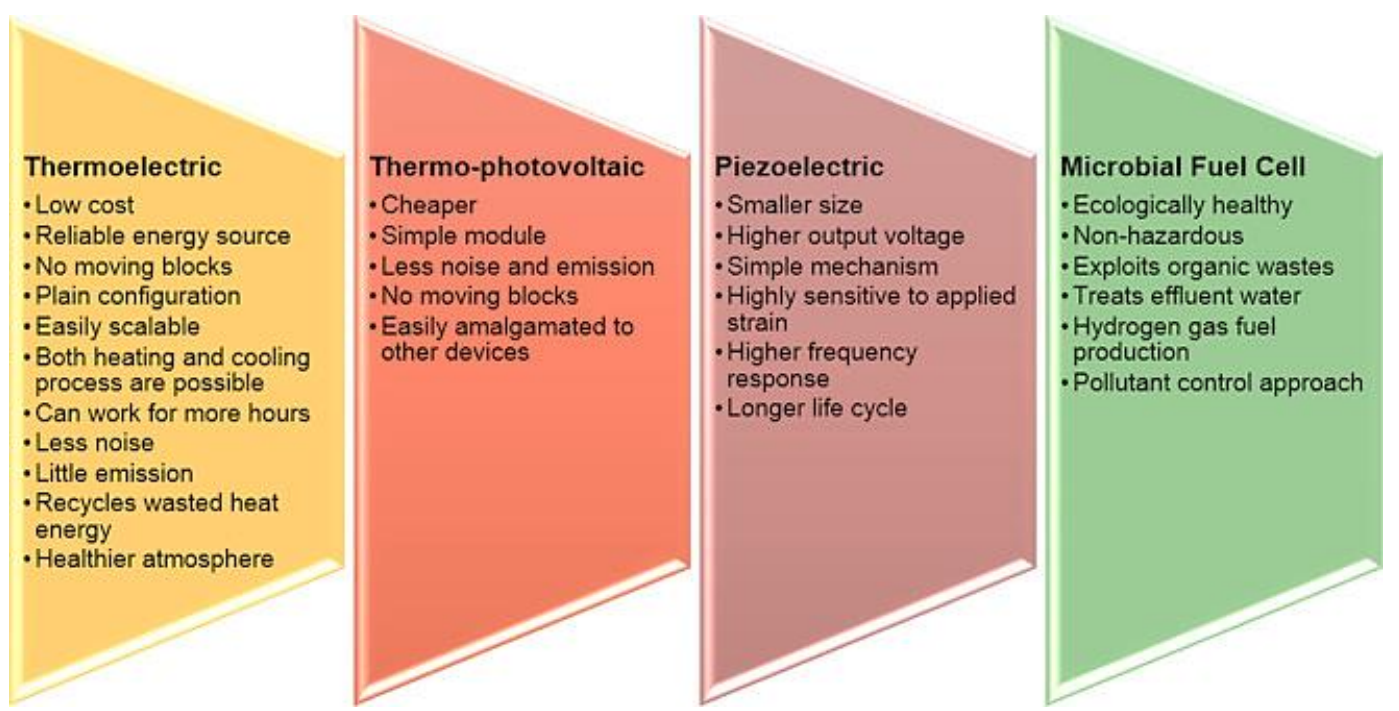

Figure 1: Positive prospects and benefits of four micro-power technologies [1].

Energy harvesting from mechanical into electrical energy include piezoelectric, electromagnetic, electrostatic and magnetostrictive or magnetoelectric conversion methods as well the use of electroactive polymers, such as dielectric elastomers and ionic polymer-metal composites [2,3]. Wasted power exists in a large number of activities including industrial machines, human activity, vehicles, structures and environmental sources and one of the goals of energy harvesting is to enable self-powered micro devices by collecting environmental energy for electronic applications ranging from structural health monitoring to medical implants [2]. Among the interesting and promising sources for recovering energy from are periodic vibrations generated by rotation machinery or engines at industrial scale or environmental sources such as wind or ocean waves [2, 4]. There are different large-scale renewable technologies used to generate energy that can be considered forms of energy harvesting, such as solar panels, wind turbines or ocean waves, which are the most common ways to convert energy nowadays [5]. In the last decade, dielectric elastomers generators have been developed to obtain energy from renewable sources, such as ocean waves, with increased efficiency for electromechanical conversion [4].

The present chapter will be devoted, to energy harvesting systems based on piezoelectric materials as well as on their applications. Up to now, the piezoelectric effect has been extensively used in different applications, such as transducers, sensors, actuators, surface acoustic wave devices and frequency control, among others $[2,3]$ and, in the scope of energy harvesting, it is increasingly explored for low power electronic systems, in the 
field of the variety of systems developed for this purpose, as illustrated in Figure 2. Piezoelectric materials generally possess crystalline structures with dipolar moments that vary/reorient under the application of mechanical solicitations, leading to a voltage variation that can be harvested by storing it in rechargeable batteries or capacitors.

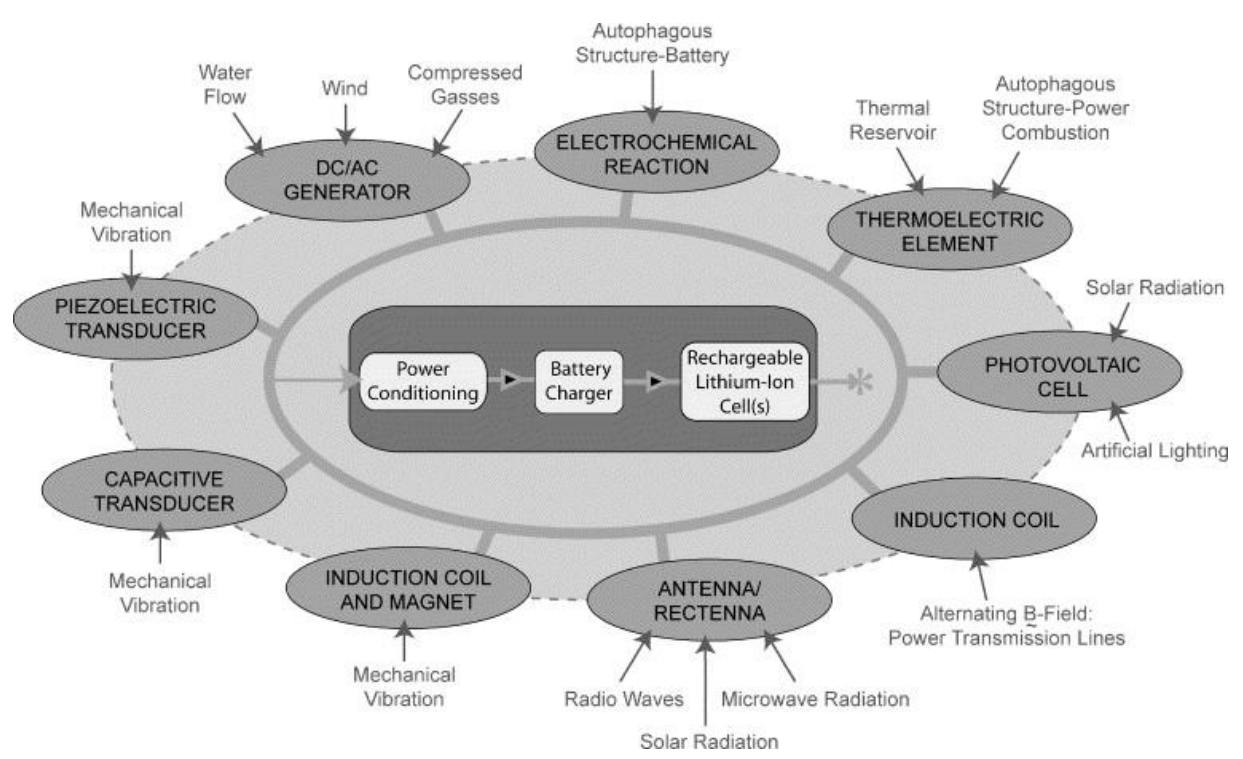

Figure 2: Various energies sources that can be converted into electrical energy for powering electric systems [6].

Piezoelectric energy harvesting remains the most widely investigated harvesting method due to its ease of application, high voltage output without requiring post-processing for voltage multiplication or bias input, high-power density and relative mature processing of the materials, which can be used to fabricate devices at different geometric scales [2]. Energy harvesting has been also extensively studied in ionic polymer metal composites, magnetostrictive materials and shape memory alloys, among others, but based on the current state of the technology, piezoelectric materials are preferred due their efficiency and reliability [7].

In this scope, piezoelectric energy harvesting from vibrational movement is one of the most popular technologies to supply energy for wireless sensor networks [8]. In a simple system, the harvester beam is located on a vibrating host structure, and the dynamic strain induced in the piezoelectric layer generates an alternating voltage output across the electrodes covering the piezoelectric layer, as shown in Figure 3. 
(a)

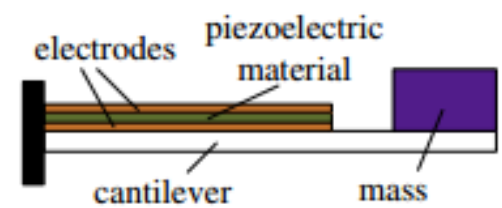

(b)

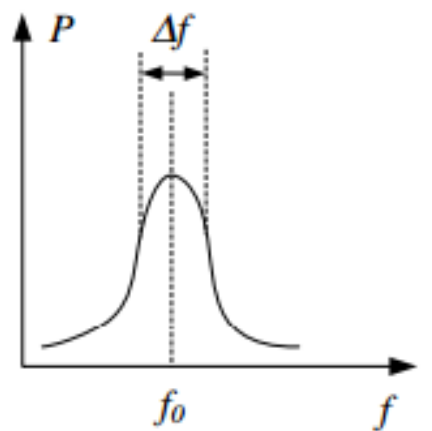

Figure 3: (a) Illustration of a piezoelectric energy harvester with a linear system of mass-cantilever structure; (b) frequency response of a typical linear energy harvester with a resonant frequency of $f_{0}$ and bandwidth of $\Delta f[9]$.

In this way and taking into account the growing field of low-power electronic technology, energy harvesting is a very attractive method for a wide range of self-powered microdevices, in particular in the area of sensors located in remote areas and difficult to access locations. Thus, when compared with conventional batteries, an autonomous power system with an embedded energy-harvesting device would be of great significance in terms of environmental friendliness and low-cost maintenance, in particular in difficultto-access environments. These low-power generators can be defined as harvesters of the available mechanical vibration from the surroundings and converting it into electrical energy and, in this scope, Table 1 shows several micro-energy harvesting sources and Table 2 shows typical acceleration and peak frequencies of potentially useful vibration sources for energy harvesting $[10,11]$.

Table 1: Energy sources available in the surrounding for harvesting electricity [11].

\begin{tabular}{|c|c|}
\hline Main sources & Type of source \\
\hline Environment & $\begin{array}{c}\text { Solar, light, wind, tide, temperature and pressure gradients, acoustic wave, } \\
\text { sound, thundering, radioactive materials, water flows }\end{array}$ \\
\hline Human body & $\begin{array}{c}\text { Blood pressure, body temperature, walking, running, arm, leg, and finger } \\
\text { motion, breathing }\end{array}$ \\
\hline Vehicles & Automobiles, aircraft, trains, turbines, tires, ships \\
\hline Structures & Building, bridges, roads, MEMS, control switch \\
\hline Industrial & Motors, generators, compressors, fans, pumps, switch-gates \\
\hline
\end{tabular}


Table 2: Acceleration and peak frequency of different vibration sources [11].

\begin{tabular}{|c|c|c|}
\hline Vibration based energy sources & Acceleration $\left(\mathbf{m} / \mathbf{s}^{\mathbf{2}}\right)$ & Peak frequency $(\mathbf{H z})$ \\
\hline Human walking & 0.4 & 2 \\
\hline Passenger automobile engine & 12200 & 200 \\
\hline Base of 3-axis machine tool & 10 & 70 \\
\hline After closing the door & 3 & 125 \\
\hline Blender machine & 6.4 & 121 \\
\hline Washing and drying machine & 3.5 & 121 \\
\hline Instrumental panel of car & 3 & 13 \\
\hline HVAC vents in buildings & $0.2-1.5$ & 60 \\
\hline Microwave oven & 2.5 & 121 \\
\hline Windows beside busy street & 0.7 & 100 \\
\hline Footbridge & - & $0.9-1$ \\
\hline
\end{tabular}

It is to notice that power transferred to a load is limited by the availability of the raw energy and the efficiency of the transducer and the conversion circuit, and that all these issues must be taken into account for the development of a proper energy harvesting system.

Due their excellent overall properties of the piezoelectric materials of single crystals, ceramics and polymer, is one of the most widely used materials due their wide band width, fast electromechanical coupling and response, relatively low power requirements and high generative forces [12]. The piezoelectric devices in the market increasesd in the last two decades, in several areas applications.

Most of the required specifications for device applications moves towards nano- and micropower energy harvesting. In the last two decades (in 1996 T. Starner theorized a computer powered by the energy of the body) several investigations allowed to stongly improve materials performance for developing energy harvesters, with specific reference to on-body applications [13]. These type of applications are particularly interesting for the next generation of wearable electronic systems, avoiding the need to charge them. On the other way, electronics miniaturization structures and devices have led to a continuous reduction in power consumption, leading to the possibility to replace batteries by energy harvesting systems in a variety of low power consumption applications.

Figure 4 shows the power consumption values of commercial off-the-shelf components, the values ranging between $100 \mathrm{nW}$ to $10 \mathrm{~mW}$, which is the typical range of the power that can be harvested from the surface of the human body [13]. 


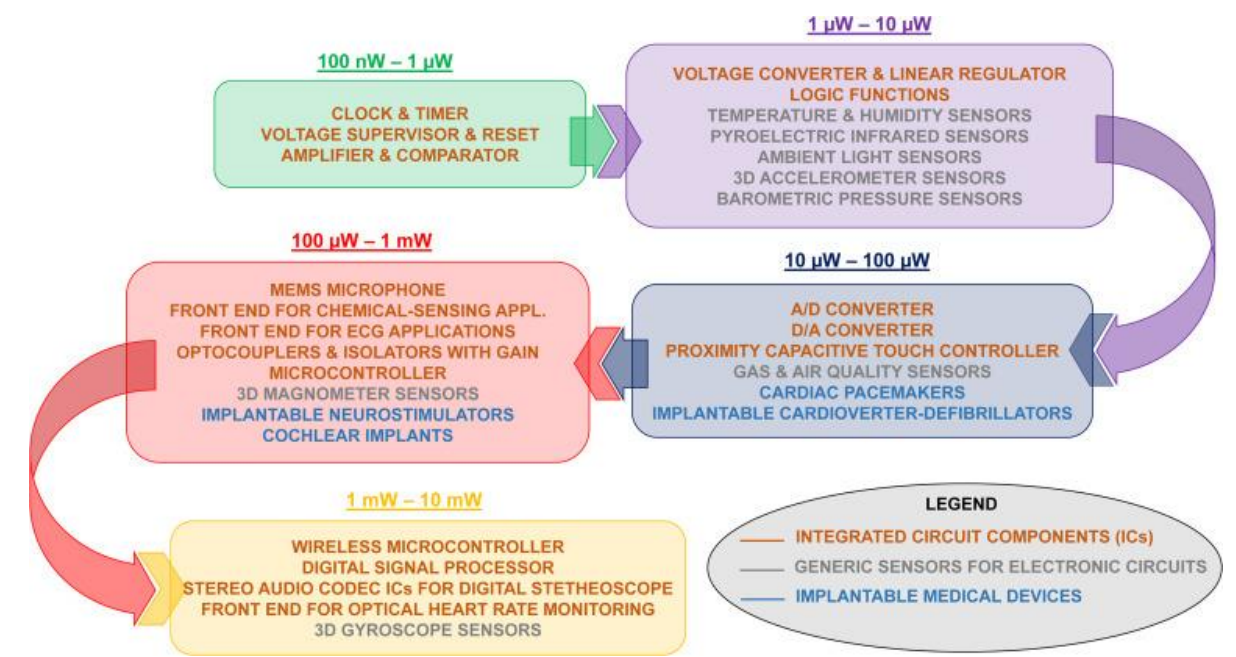

Figure 4: Average power consumed by generic integrated circuit components and sensors, as well as by implantable devices. The consumed power typically ranges from $100 \mathrm{nW}$ to $10 \mathrm{~mW}$. The colors codify the power consumption ranges: green, $100 \mathrm{nW}-1 \mu \mathrm{W}$; violet, $1-10 \mu \mathrm{W}$; blue, $10-100 \mu \mathrm{W}$; red, $100 \mu \mathrm{W}-1 \mathrm{~mW}$; yellow, 1-10 mW [13].

\section{Background and Fundamentals}

This chapter presents the main theoretical ideas related to the piezoelectric effect and related concepts in different piezoelectric structures and devices.

\subsection{Structural considerations}

Crystalline structure can be classified into 32 crystallographic point groups. Of all 32 crystallographic point groups, 11 classes have inversion symmetry and the remaining 21 classes have no inversion symmetry, a necessary requirement for the existence of piezoelectricity. One of these 21 classes with no inversion symmetry, possesses other combined symmetry elements, thus rendering no piezoelectricity. In this way, there are 20 crystallographic point groups with would exhibit piezoelectric effect. From these 20 classes, in half of them the polarization can be induced by a mechanical stimulus, while the remaining 10 classes possess spontaneous polarization and thus can show piezoelectric as well pyroelectric effects. There is a subgroup within these 10 classes that possesses spontaneous and reversible polarization under an electric field: ferroelectric effect. This subgroup can thus exhibit all three effects, ferro-, piezo-, and pyroelectric. Thus, the classification of the crystals show that all ferroelectric crystals possess piezoand pyroelectric properties. All pyroelectric crystals also show piezoelectric properties, but not all are ferroelectric. Piezoelectric crystals can show both or just one of them. 
Piezoelectricity can be described as the capability of certain materials to develop an electrical voltage under the application of a certain mechanical stress, and will be described later in more detail. Pyroelectricity represents the property of some crystals to develop an electrical potential when heated. As the crystal temperature changes, it will cause changes in the spontaneous polarization and an electrical potential is established [14]. The energy conversion efficiency of the pyroelectric device is related to the electrical energy outputs over thermal energy input. Pyroelectricity was firstly described in the $18^{\text {th }}$ century but the first reference is in $314 \mathrm{BC}$ in tourmaline [14]. The theory of the pyroelectric effect was formulated at the beginning of the $18^{\text {th }}$ century by Louis Lemery [14].

Ferroelectric materials (a subgroup of pyroelectric crystals) present a spontaneous polarization that can be reversed under application of an external electric field, lower than the dielectric breakdown of the material. Ferroelectricity was discovered in 1921 in Rochelle salt. Following these discover the phenomenon was observed in several families materials including hydrogen bonded crystals, oxides, polymers and liquid crystals [15]. There are four categories groups of the ferroelectric materials, being the perovskite structure $\left(\mathrm{ABO}_{3}\right)$ the most important one, followed by the bismuth layer structure, the tungsten bronze and the pyroclore group [16]. Most ferroelectric materials show a phase transition at the so called Curie temperature: below the Curie temperature the material is ferroelectric and at that temperature the material undergoes a structural phase transition to a paraelectric phase. It is commonly understood that the ferroelectric structure of a crystal material is developed by a small distortion of the paraelectric structure such that the lattice symmetry in the ferroelectric phase is always lower than that in the paraelectric phase [17].

\subsection{Piezoelectric effect}

The word piezoelectricity derives from Greek piezein that means "to press". Piezoelectricity can be described as the capability of certain materials to develop an electrical voltage under the application of a certain mechanical stress. Crystalline materials composed by polar structures without a center of symmetry will show piezoelectric properties. The piezoelectric effect is historically divided into direct and the inverse piezoelectric effect and describes the ability of a material to transform mechanical energy into electrical energy or the inverse mechanism, transform electrical signals into mechanical variations (as exemplified in Figure 5). For the direct piezoelectric effect, 
when the piezoelectric material is subjected to external strain, the electric dipoles in the crystal are oriented such that the crystal develops positive and negative charges on opposite surfaces, resulting in an electric field within the material. When an electric field is applied to the piezoelectric material (inverse piezoelectric effect), asymmetric displacements of anions and cations will take place that cause considerable net deformation of the crystal, the resulting strain being directly proportional to the applied electric field unlike electrostrictive materials in which the strain is proportional to the square of the electric field. Depending on the polarity of the applied field, the strain of the piezoelectric material is extensive or compressive.

The direct effect was discovered by the brothers Pierre and Jacques Curie in 1880 [18], while the inverse effect was deduced mathematically from the fundamental principles of thermodynamics by Lippmann in 1881 and then confirmed experimentally by the Curie brothers in the same year [19].

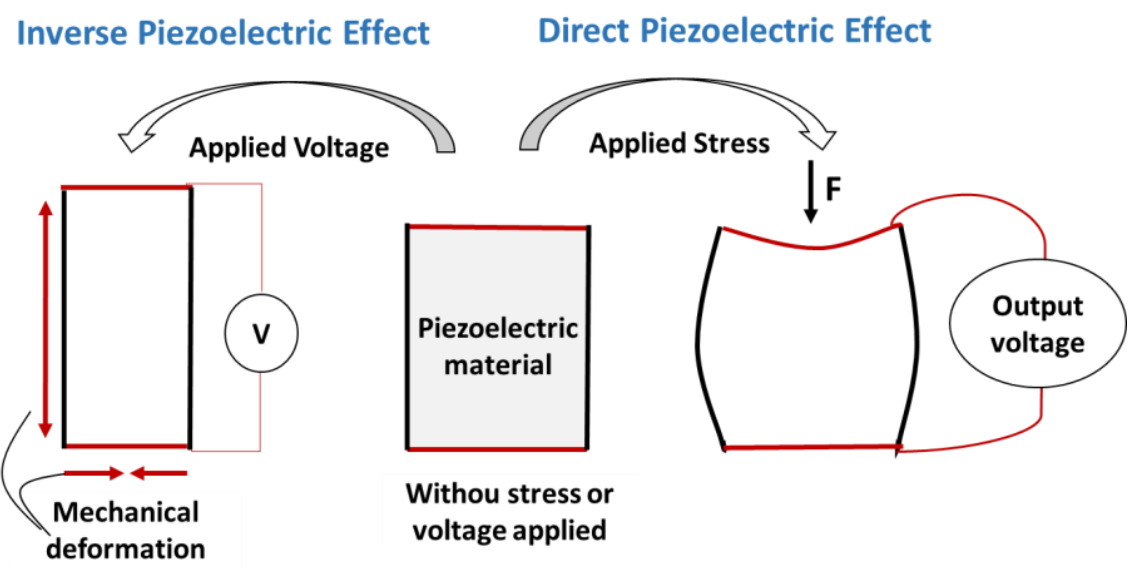

Figure 5: Schematic representation of the direct and inverse piezoelectric effects.

The direct and inverse piezoelectric effects are described by the following constitutive equations [19]:

$$
\begin{aligned}
& D_{i}=d_{i k l} T_{k l}+\varepsilon_{i k}^{T} E_{k} \\
& S_{i j}=s_{i j k l}^{E} T_{k l}+d_{k i j} E_{k}
\end{aligned}
$$


In equations (4) and (5) subscripts $i, j, k$ and $l$ take values of 1, 2, and 3. The strain and stress tensors are $S$ and $T$, respectively and $D$ and $E$ are the electric displacement and electric field vectors, respectively. Further, $s$ is the elastic compliance matrix evaluated at a constant electric field, $d$ are the piezoelectric strain coefficients and $\varepsilon$ the permittivity values evaluated at constant stress. In both equations $d$ represents the charge variation induced by a given force in the absence of an electric field (short circuit electrical condition), or the deflection caused by an applied voltage in the absence of an applied force (stress-free mechanical condition) [20] and illustrated in Figure 6.

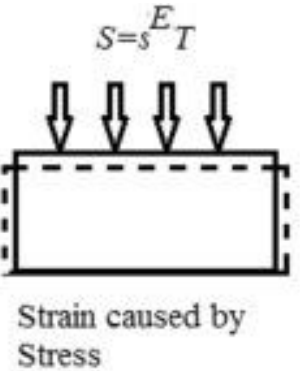

$D=\varepsilon^{T} E$

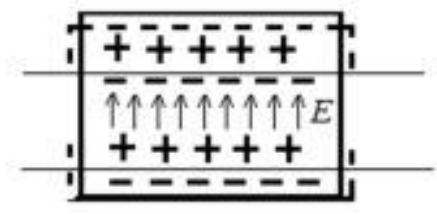

Extra electric displacement via internal electric field based on material's dielectric permittivity.

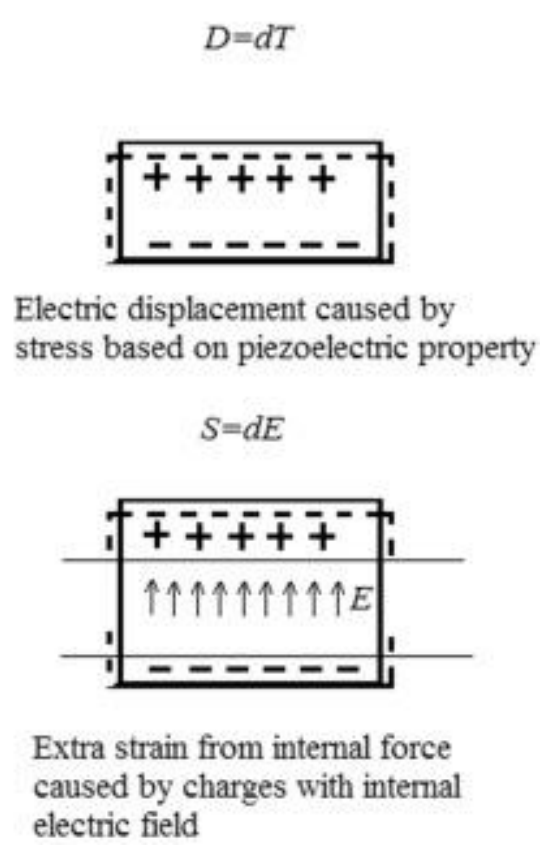

Figure 6: Schematic representation of the meaning of the parameters involved in the piezoelectric coupling equations 1 and 2 [21].

The direct piezoelectric effect is mainly responsible for the application of the material as a sensor and the inverse piezoelectric effect is accountable for its ability for act as an actuator $[15,22]$.

There are a wide range of commercial applications taking advantage of the piezoelectricity of the materials. The main application areas are communications and control, industry, health and consumer electronics among others applications. Some particular examples are radio, television or radars, transducers, sensors or actuators, sonars, pumps or motors. More recently, these materials are implemented in microelectromechanical (MEMS) devices and microoptomechanical (MOMS) devices, smarts structures or biomimetic devices. 


\subsection{Piezoelectric coefficients and modes}

The piezoelectric coefficients $d$ are represented by unit of meters per volt or coulombs per newton. The most standard and used piezoelectric coefficients are $d_{31}$ and $d_{33}$, respectively known as transverse and longitudinal coefficient. The transverse coefficient describes the electric polarization generated in a direction perpendicular to the applied stress in material. The longitudinal coefficient describes the electric polarization generated in the same direction of the applied stress [23].

\subsection{The electromechanical coupling coefficient}

The electromechanical coupling coefficient, $k$, represents the conversion efficiency between electrical and mechanical energy (and vice versa), being closely related to the bandwidth of resonant devices and it is expressed as [23].

$$
k_{i j}=\frac{d_{i j}}{\sqrt{\varepsilon_{i i}^{\sigma} S_{j j}^{E}}}
$$

The coupling factor is related to the different vibration modes and can be experimentally measured directly from the resonance and anti-resonance frequencies observed in the piezoelectric elements.

\subsection{Mechanical quality factor}

The mechanical quality factor, $Q$, is related to the power loss over the energy stored in the system. For a piezoelectric resonator, the mechanical quality factor characterizes the resonator's bandwidth:

$$
Q=f_{r} / \Delta f
$$

where $f_{r}$ is the resonant frequency and $\Delta f$ the frequency difference at $-3 \mathrm{~dB}$ of the maximum admittance [15]. The single crystal efficiency of the mechanical quality factor decreases significantly with the existence of domain wall motion/polarization rotation. 
Other factors which affect the mechanical quality factor are the polarization rotation angle, domain size and state (single domain or multidomains).

\section{Materials used in piezoelectric transducer for Energy Harvesting}

Piezoelectric materials can be natural or synthetic, the most common natural piezoelectric material being quartz $\left(\mathrm{SiO}_{2}\right)$. Synthetic piezoelectric materials are more efficient when compared to natural, and the most used ones are ceramic materials.

Natural piezoelectric materials can be divided into two main groups: the mineral group, including quartz, Rochelle salt, topaz and tourmaline, among others, and organics groups, represented by silk, wood, enamel, dentin, bone, hair and rubber, among others [18].

These piezoelectric materials when subjected to mechanical loads and/or vibration (usually in the 31 or 33 mode, exemplified in Figure 7) produce a variation of the surface electrical charge. The voltage and charge produced depend on the overall piezoelectric properties of the material, the mechanical loading and the structure of the device application.

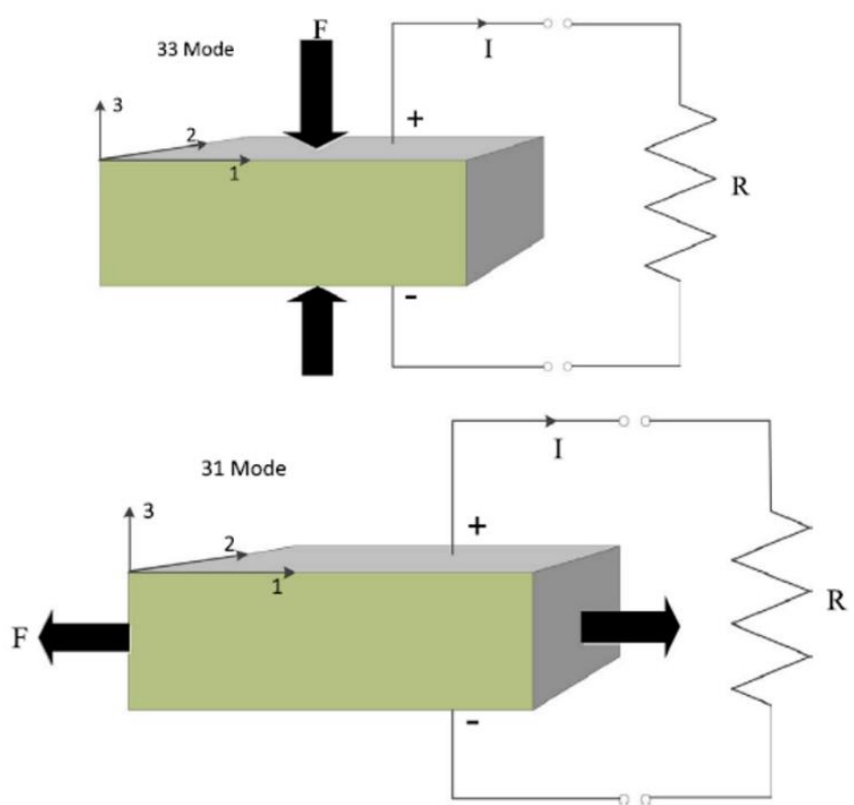

Figure 7: Illustration of two operational modes of a piezoelectric material: 33-mode (up) and 31-mode (down) [24].

Piezoelectric materials can be more precisely divided into four different categories based on their structural characteristics: single crystals, ceramics, polymers and their composites, being the later a combination of piezoelectric ceramics or single crystals embedded into a piezoelectric or non-piezoelectric matrix. 
Most piezoelectric single crystals and ceramics used for up to date for energy harvesting applications are also ferroelectrics. The ferroelectric single crystals, ceramics, and composites show much larger piezoelectric response that polymers, the latter showing, however, the ability to sustain much higher strains due to their intrinsic flexibility. Nowadays, an increasing number of studies are being devoted to perovskite nanostructures including nanowires, nanorods, nanotubes, nanobelts, nanowhiskers and nanoplates, which are expected to open up new applications in nanoelectronics and selfpowered nanodevices [25]. Develop suitable and scalable processing approaches to develop the piezoelectric materials with novel structures and tailored properties is one of the main scientific and technological challenges of the near future.

The selection of a certain piezoelectric material for a specific energy harvesting applications is determined not only by the piezoelectric properties, but also by the specific design requirements of the energy harvesting unit, such as the application frequency, the available volume, and the form in which mechanical energy is fed into the system, among others.

Strictly from the materials perspective, the important properties of piezoelectric materials for energy harvesting applications include the piezoelectric strain constant $d$ (induced polarization per unit stress applied, or induced strain per unit electric field applied), piezoelectric voltage constant $g$ (induced electric field per unit stress applied), electromechanical coupling factor $k$ (an indication of the mechanical to electrical energy conversion efficiency), mechanical quality factor $Q$ (degree of damping; lower value indicates higher damping), and dielectric permittivity $\varepsilon$ (the ability of the material to store charge).

\subsection{Single Crystals}

Piezoelectric nanogenerators have attracted extensive attention as they can harvest ubiquitous mechanical energies from ambient environment continuously, for selfpowered nanosystems that enable continuous operations of implantable biodevices, micro-electrochemical systems, wireless sensors and portable/wearable electronics, without the trouble of charging and replacing micro-power sources.

Piezoelectric single crystal (also known as piezocrystals) were the first discovered piezoelectric materials but they were largely overcome by piezoelectric ceramics in practical applications. The most interesting piezoelectric crystals with technical relevance are shown in Table 3, together with some of their relevant properties. 
Quartz and lithium niobate are the most traditional single crystals; however, they exhibit inferior piezoelectric performance compared to lead zirconate titanate (PZT) ceramics. Quartz is silicon dioxide $\left(\mathrm{SiO}_{2}\right)$ crystalized in hard, glass-like, six-sided prisms. It is intrinsic properties change with temperature, losing the piezoelectric properties above $573{ }^{\circ} \mathrm{C}$ (Curie temperature). The crystal structure showing piezoelectric response is called $\alpha$-quartz and the $\beta$-quartz do not present piezoelectric properties. Lithium niobate $\left(\mathrm{LiNbO}_{3}\right)$ has the $\mathrm{ABO}_{3}$ chemical formula (as the most commercial piezoceramics barium titanate $\left(\mathrm{BaTiO}_{3}\right)$ or PZT). It was discovered in 1949 and presents very low losses and very high longitudinal propagation speed, being particularly appropriate for highfrequency applications [26]. Furthermore, it presents very high Curie temperature, $T_{c}$ $\approx 1200{ }^{\circ} \mathrm{C}$, ideal for high temperature applications [26]. $\mathrm{LiNbO}_{3}$ single crystals produced commercially are typically grown using the Czochralski technique [27].

Rochelle salt $\left(\mathrm{NaKC}_{4} \mathrm{H}_{4} \mathrm{O}_{6}-4 \mathrm{H}_{2} \mathrm{O}\right)$ is sodium potassium tartrate tetrahydrate with four molecules of water can show a large piezoelectric effect under specific conditions of temperature and humidity [24]. Values of humidity lower than $35 \%$ and higher than $85 \%$ at room temperature leads Rochelle salt to evaporate and absorb the moisture, respectively, and begins to dissolve [24]. The range of optimum operating temperature is between $-18{ }^{\circ} \mathrm{C}$ and $24{ }^{\circ} \mathrm{C}$ [24]. It is an important crystal in the discovery of the piezoelectric phenomenon but does not present suitable properties for being used in commercial applications due to their intrinsic properties.

Lead magnesium niobate $\left(\mathrm{Pb}\left(\mathrm{Mg}_{1 / 3} \mathrm{Nb}_{2 / 3}\right) \mathrm{O}_{3}\right)$ or $\mathrm{PMN}$ exhibits high dielectric constant, broad dielectric maxima at $T_{c}$, with low sintering temperature. Synthesis of this family of compounds has, however, been found to be rather hard because of the pyrochlore phase formation mainly from the loss of $\mathrm{PbO}$ during sintering [28]. The stable pyrochlore phase in PMN is also known to reduce the dielectric constant of the material. Alternative preparation method can improve the piezoelectric properties of the PMN [28], leading to dielectric constant as high as $\varepsilon \approx 28000$.

Lead zinc niobate $\left(\mathrm{Pb}\left(\mathrm{Zn}_{1 / 3} \mathrm{Nb}_{2 / 3}\right) \mathrm{O}_{3}\right)$ or $\mathrm{PZN}$ is a typical relaxor ferroelectric material with perovskite structure. PZN-based single crystals exhibit outstanding dielectric, electrostrictive and optical properties, making them a good candidate for applications as sensors and actuators. PZN near the morphotropic phase boundary (MPB) at room temperature shows large dielectric and piezoelectric constants and higher electromechanical coupling coefficient than the PZT family of ferroelectrics [29]. On the 
other hand, it is difficult obtain PZN with a perovskite structure by conventional processing methods [30].

Based on the development of relaxor-PT (relaxor- lead titanate) system in ceramic engineering, the growth of the corresponding single crystals has also been studied bearing in mind their excellent piezoelectric properties and high Curie temperatures for applications in harsh environments.

An interesting family of single crystal piezoelectric materials is lead magnesium niobate doped with lead titanate, ${ }_{(x)} \mathrm{Pb}\left(\mathrm{Mg}_{1 / 3} \mathrm{Nb}_{2 / 3}\right) \mathrm{O}_{3-(1-x)} \mathrm{PbTiO}_{3}$ (PMN-PT) [26]. This relaxorPT family, classified as having tetragonal symmetry, has good dielectric and piezoelectric properties as a result of enhanced polarizability arising from the coupling near the MPB, shown in Figure 8.

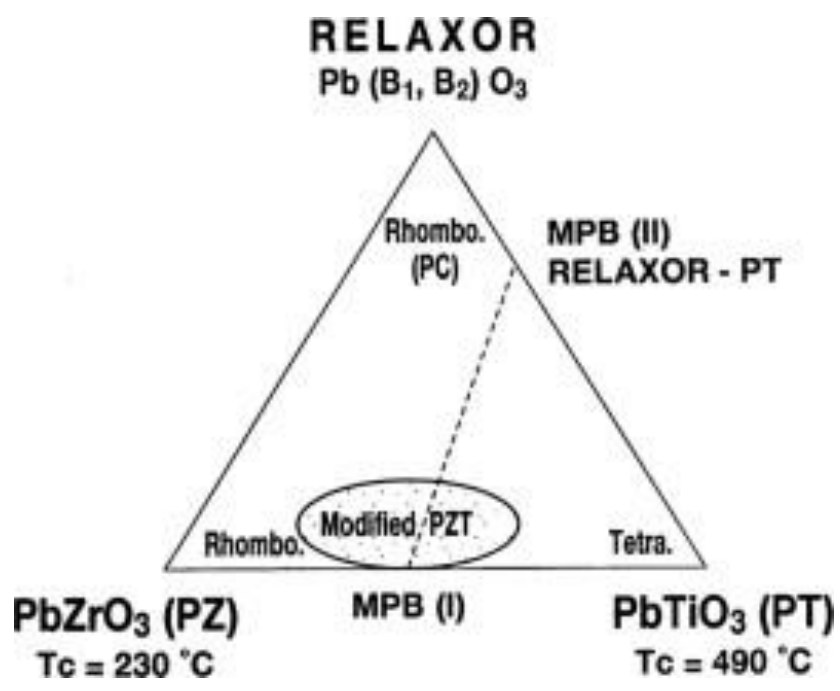

Figure 8: Ternary diagram of MPB (I) in PZT and MPB (II) in relaxor-PT systems [27].

Somewhat similar to PZT in many characteristics, its particularly attractive properties are its very high coupling coefficient, $k_{33} \approx 0.9$, and piezoelectric strain coefficient, $d_{33} \approx 1400$ $\mathrm{pm} . \mathrm{V}^{-1}$ [26], combined with a relatively high value for the piezoelectric voltage coefficient. As negative issues, PMN-PT has a low Curie temperature, $T_{c} \approx 130{ }^{\circ} \mathrm{C}$ [26]. Together with the relatively high mechanical losses, there is a significant limitation of it is potential applications where elevated temperatures or high average output ultrasound intensities are required. To overcome this problem, research is under way to extend the binary PMN-PT material system to ternary systems and doped ternary systems. These properties lead to some loss in piezoelectric performance but better mechanical properties and thermal stability. 
The ternary single crystal $\mathrm{Pb}\left(\mathrm{In}_{1 / 2} \mathrm{Nb}_{1 / 2}\right)-\mathrm{Pb}\left(\mathrm{Mg}_{1 / 3} \mathrm{Nb}_{2 / 3}\right)-\mathrm{PbTiO}_{3}(\mathrm{PIN}-\mathrm{PMN}-\mathrm{PT}$ ) shows higher Curie temperature than PMN-PT and is grown from the melt by the Bridgman method [27]. The other interesting properties remains similar to PMN-PT. These kind of ternary single crystal are increasingly being used for ultrasound transducer applications. The development of synthetic single crystal such as PZN-PT (lead zinc niobate-lead titanate) and PMN-PT near the MPB composition leads to excellent piezoelectric properties with electric field-induced strains exceeding $1 \%$ and electromechanical coupling larger than $90 \%$ (compared to the values of $0.1 \%$ and $70-75 \%$, respectively, for the PZT ceramics) [21]. Thus, the piezoelectric properties of the PZN-PT single crystal depends on the PZN/PT ratio in crystal composition, which must be taken into account to properly tuning materials functional response [31].

Strong research efforts have led to the development of highly efficient processing methods, including high-temperature solutions and/or congruent melt, for producing PZN-PT and PMN-PT single crystals.

Aluminum nitride (AIN) is a piezocrystal with interesting properties for applications. In particular, AlN has the same crystallographic symmetry as piezoceramic PZT and a behavior somewhat similar to $\mathrm{LiNbO}_{3}$ [26]. However, it is distinguished by its realization as a highly inert, crystalline thin film, leading to its use in surface acoustic wave devices. Lithium tantalate $\left(\mathrm{LiTaO}_{3}\right)$ has the same chemical structure than $\mathrm{LiNbO}_{3}$ and $\mathrm{AlN}$. AlN shows a high Curie temperature $\left(600^{\circ} \mathrm{C}\right)$, suitable linear characteristics are observed in electro-optic and piezoelectric responses at room temperature.

In addition to the aforementioned materials, there are other single crystals with interesting piezoelectric properties but due their rarity, manufacturing cost or stability are not used in energy harvesting systems. An example is tourmaline that is a semiprecious stone, also known as "Ceylon Magnet". The piezoelectric applications of tourmaline involve the measuring of hydrostatic pressures and very high frequency applications. The shortage and expensive cost of large-size crystals makes the tourmaline inappropriate to commercial applications. Ammonium dihydrogen phosphate (ADP) was discovered in 1930s and was used as a substitute for Rochelle salt as underwater sound transducer. ADP is stable in the presence of water, can operate in environments up to $100{ }^{\circ} \mathrm{C}$ [32], can be grown commercially and show better mechanical properties than Rochelle salt. Ethylene diamine tartrate (EDT) was discovered in 1930s having the advantage of a wide gap between their resonant and antiresonant frequencies. EDT was used to substitute quartz as low-frequency filters in military communications. On the other hand, EDT shows 
higher temperature sensibility than quartz for high-frequencies, requiring a suitable control of the operating temperature. Finally, dipotassium tartrate (DKT) is a synthetic crystal with similar chemical structure than Rochelle salt (the sodium atom has been replaced by a potassium atom) and exhibits no tendency to dehydrate below $80{ }^{\circ} \mathrm{C}$. It has similar properties than EDT but the DKT crystals are more difficult to grow, hindering their incorporation into commercial applications.

The main piezoelectric properties of the most used single crystals in piezoelectric systems are presented in Table 3.

Table 3: Main piezoelectric properties of the single crystal used in energy harvesting and related applications.

\begin{tabular}{|c|c|c|c|c|c|c|}
\hline Materials & $\begin{array}{c}\text { PE charge } \\
\text { constant } \\
d_{33}(p C / N)\end{array}$ & $\begin{array}{c}\text { PE charge } \\
\text { constant } \\
d_{31}(p C / N)\end{array}$ & $\begin{array}{c}\text { EM } \\
\text { coupling } \\
\text { factor } k_{33}\end{array}$ & $\begin{array}{c}\text { Dielectric } \\
\text { constant } \\
\varepsilon_{\mathrm{r}} \\
\end{array}$ & $\begin{array}{c}\boldsymbol{T}_{\mathbf{c}} \\
\left({ }^{\circ} \mathbf{C}\right)\end{array}$ & Ref. \\
\hline Quartz & 2 & - & - & 4.5 & - & [31] \\
\hline $\mathrm{LiNbO}_{3}$ & 49 & - & 0.47 & 47 & 1140 & {$[27,33]$} \\
\hline Rochelle salt & - & 275 & 0.65 & & $<24$ & [17] \\
\hline PMN & $<2800$ & - & $0.91-0.95$ & $>5400$ & $<150$ & {$[15,28]$} \\
\hline PZN & - & - & - & 950 & - & [15] \\
\hline PMN-PT & 1620 & -760 & 0.93 & 7000 & $<155$ & $\begin{array}{c}{[15,27,} \\
34]\end{array}$ \\
\hline PIN-PMN-PT & 2742 & -1337 & 0.95 & 7244 & 200 & $\begin{array}{c}{[27,34,} \\
35]\end{array}$ \\
\hline PMN-PZT & 1530 & -718 & $0.92-0.93$ & $>4850$ & $\approx 216$ & {$[15,35]$} \\
\hline PZN-PT & 2200 & -1075 & 0.94 & 5100 & - & {$[15,35]$} \\
\hline AlN & 5.1 & -2 & 0.38 & & $>2000$ & [36-38] \\
\hline $\mathrm{LiTaO}_{3}$ & 14.6 & 7.28 & 0.32 & $44-47$ & 620 & {$[39,40]$} \\
\hline
\end{tabular}

Single crystals have improved piezoelectric properties (piezoelectric coefficients or electromechanical coupling) compared to ceramics crystals. On the other hand, piezoceramics are easier and cheaper to produce than single crystals.

\subsection{Ceramics}

Piezoelectric ceramics (or piezoceramics) are the most widely used piezoelectric materials in device applications. The most important ferroelectric oxides for piezoelectric 
ceramics are restricted to perovskite-type, tungsten bronze-type, and bismuth layerstructured compounds.

The discovery of piezoelectric ceramics with improved properties, such as barium titanate $\left(\mathrm{BaTiO}_{3}\right)$ and lead zirconate titanate (PZT) in the 1940s and 1950s, respectively, increased their commercialization for practical applications [26]. These families of materials exhibited very high dielectric and piezoelectric properties and can be tailored to improve their behavior to appropriate and specific responses, also by the use of specific dopants. These materials bring the benefit of relatively high electromechanical coupling factor and piezoelectric constant and, therefore, display improved bandwidth and sensitivity. To date, PZT is one of the most widely used piezoelectric materials, the vast majority of ultrasonic transducers using polycrystalline piezoelectrics based on the composition PZT. However, considering the toxicity of lead and its compounds and the environmental protection rules for waste disposal of products, there is a general awareness for the development of lead-free materials as evidenced from the legislation passed by the European Union in this effect.

Both ceramics, $\mathrm{BaTiO}_{3}$ and PZT, are based on the perovskite structure $\left(\mathrm{ABO}_{3}\right)$ shown in Figure 10. The structure is characterized by corner-sharing oxygen octahedra linked together in a regular cubic array with smaller cations (Ti, $\mathrm{Zr}, \mathrm{Sn}, \mathrm{Nb}$ ) occupying the central octahedral B-site, and larger cations $(\mathrm{Pb}, \mathrm{Ba}, \mathrm{Sr}, \mathrm{Ca}, \mathrm{Na})$ filling the interstices between octahedra in the larger A-site (Figure 9).

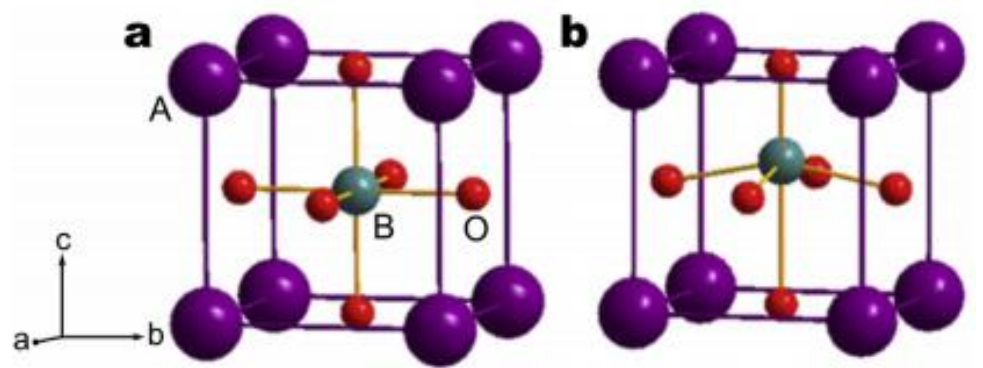

Figure 9: Schematic representation of the $\mathrm{ABO}_{3}$ perovskite structure of (a) cubic and (b) tetragonal phases [25].

There are three classes of perovskite compounds including $\mathrm{A}^{\mathrm{I}} \mathrm{B}^{\mathrm{V}} \mathrm{O}_{3}, \mathrm{~A}^{\mathrm{II}} \mathrm{B}^{\mathrm{IV}} \mathrm{O}_{3}$ and $\mathrm{A}^{\mathrm{III}} \mathrm{B}^{\mathrm{III}} \mathrm{O}_{3}$ where the Roman numerals represent the chemical valence of the cations (Table 4), together with some representative examples [25]. 
Table 4: Examples of perovskite structures [25].

\begin{tabular}{|c|c|}
\hline Perovskite structures & System examples \\
\hline $\mathrm{A}^{\mathrm{I}} \mathrm{B}^{\mathrm{V}} \mathrm{O}_{3}$ & $\mathrm{KNbO}_{3}, \mathrm{NaNbO}_{3},(\mathrm{~K}, \mathrm{Na}) \mathrm{NbO}_{3}$ \\
\hline $\mathrm{A}^{\mathrm{II}} \mathrm{B}^{\mathrm{IV}} \mathrm{O}_{3}$ & $\mathrm{PbTiO}_{3}, \mathrm{PbZrO}_{3}, \mathrm{~Pb}(\mathrm{Zr}, \mathrm{Ti}) \mathrm{O}_{3}, \mathrm{BaTiO}_{3}$ \\
\hline $\mathrm{A}^{\mathrm{III}} \mathrm{B}^{\mathrm{II}} \mathrm{O}_{3}$ & $\mathrm{BiFeO}_{3}, \mathrm{BiScO}_{3}$ \\
\hline
\end{tabular}

PZT ceramic is based in lead titanate $\left(\mathrm{PbTiO}_{3}\right.$ or PT) that is a ferroelectric material with a high phase transition temperature. It is widely used in most of transducer and actuator for high temperature and frequency applications due to its stable pyro- and piezoelectric properties [41]. The piezoelectric PZT ceramic solid-solution is a representative piezoelectric material used in many electronic devices due its superior piezoelectric coefficients $\left(d_{33} \approx 300-1000 \mathrm{pC} / \mathrm{N}\right)$ [3]. The crystalline symmetry of this solid-solution system is determined by the zirconate $(\mathrm{Zr})$ content. Lead titanate also a tetragonal ferroelectric phase with a perovskite structure. Increasing Zr content in PZT, leads to a structural changes from a tetragonal to a rhombohedral ferroelectric phase in the vicinity of the $\mathrm{Zr} / \mathrm{Ti}=52 / 48$ [25]. The MPB delimits both phases with both tetragonal and rhombohedral phases coexisting together [42].

MPB compositions show high dielectric and piezoelectric properties as a result of enhanced polarizability, arising from the coupling between two equivalent energy states, that is, the tetragonal and rhombohedral phases, allowing optimum domain reorientation during the poling process $[42,43]$.

Lead zirconate titanate $\left(\mathrm{Pb}\left(\mathrm{Zr}_{1-\mathrm{x}} \mathrm{Ti}_{\mathrm{x}}\right) \mathrm{O}_{3}\right)$ is a solid solution of ferroelectric $\mathrm{PbTiO}_{3}\left(T_{c}=\right.$ $\left.490{ }^{\circ} \mathrm{C}\right)$ and antiferroelectric $\mathrm{PbZrO}_{3}\left(T_{c}=230^{\circ} \mathrm{C}\right)$. $\mathrm{PZT}$ properties depend on the ratio of $\mathrm{Zr} / \mathrm{Ti}$, being $\mathrm{Pb}\left(\mathrm{Zr}_{0.52} \mathrm{Ti}_{0.48}\right) \mathrm{O}_{3}$ the one with large piezoelectric properties to be applied in transducer applications (with structure and main properties showed in Figure 10 and Table 5). Furthermore, it has a high $T_{c}=390{ }^{\circ} \mathrm{C}$ which allows piezoelectric devices to be operated at relatively high temperatures and high electromechanical coupling coefficients [44]. Further, PZT can be easily polarized. PZT materials can be doped with donor, acceptor or isovalent ions, with a strong variation of their properties [43]. Donor doping creates "soft" PZT, such as PZT-5, as the Pb vacancies allow domain motion. These ceramics are used in high sensitivity applications as hydrophones or loudspeakers [43]. Acceptor doping leads to "hard" PZT, such as PZT-8, as the oxygen vacancies will restrict the motion of the domain walls. High-power devices such as sonars or ultrasonic transducer are the typical applications [43]. Isovalent doping changes ions of the same 
valence and may result in a decrease of the Curie temperature and an increase of the dielectric constant [43].

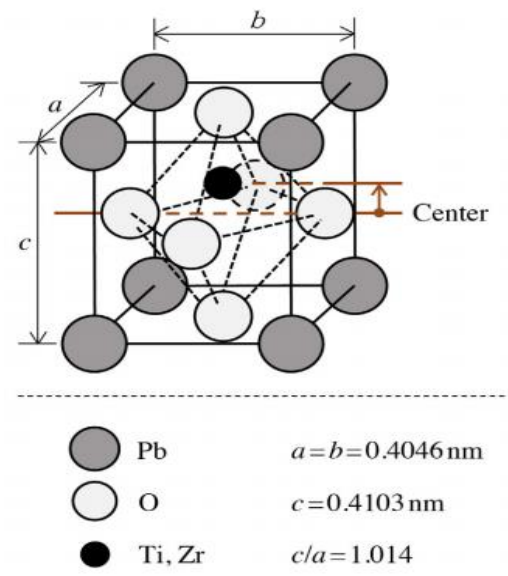

Figure 5: Tetragonal lattice structure of the PZT ceramic [45].

Table 5: Piezoelectric properties of the PZT ceramic [45].

\begin{tabular}{|c|c|}
\hline Effective elastic constant $\left(\varepsilon_{33}^{E}\right)$ & $53 \mathrm{GPa}$ \\
\hline EM coupling coefficient $\left(\mathrm{k}_{33}\right)$ & 0.71 \\
\hline Piezoelectric constant $\left(\mathrm{d}_{33}\right)$ & $472 \mathrm{pm} / \mathrm{V}$ \\
\hline Permittivity $\left(\varepsilon_{33}\right)$ & $22.7 \mathrm{nF} / \mathrm{m}$ \\
\hline
\end{tabular}

PZT in ternary solid solutions with another perovskite phase has been investigated intensively with interesting results, including higher Curie temperatures or dielectric properties. PZT-4, PZT-5A (-5H) and PZT-8, known as soft, semi-hard and hard PZT, respectively, are examples of intense studies on the properties of these piezoceramic materials [43].

Replacing the lead $(\mathrm{Pb})$ sites with trivalent or monovalent (donor or acceptor) impurities affects the piezoelectric properties due the generated vacancies. Among the different investigated dopants, the donor impurity lanthanum it is found to improve the piezoelectric and dielectric properties of PZT. Adding lanthanum in different concentrations and at different $\mathrm{Zr} / \mathrm{Ti}$ ratiosallows to produce lead lanthanum zirconate titanate (PLZT) ceramics, which exhibits a variety of ferroic phases and cover all compositional aspects of the piezo-, pyro- and ferroelectric, ceramic properties. Thus, commercial PLZT shows higher piezoelectric coefficients and dielectric constant values 
than pristine PZT [46]. On the other hand, the addition of lanthanum decreases the stability of the ferroelectric phases in favor of the paraelectric and antiferroelectric phases, also decreasing the Curie temperature with increasing lanthanum in PLZT. At a critical concentration of lanthanum in PZT, the temperature of the stable ferroelectric polarization is reduced to slightly below room temperature [16]. In this way, the result is a material that is non-ferroelectric and cubic in its virgin state. Further, the phase diagram also shows a region of diffuse, metastable relaxed phases that can be electrically induced to a ferroelectric phase along the ferro- to paraelectric phase boundary [16].

As previously indicated, $\mathrm{Pb}$ is toxic and lead-free piezoelectric perovskites are being intensively investigated to developing lead-free piezoelectrics in response to the request for environmental protections. Lead-free alternatives are studied for applications in memory devices and microelectromechanical systems (MEMS) [25, 42, 47, 48]. These materials have recently been attracted much attention due to their excellent ferro- and piezoelectric properties together with their environmental friendliness [49]. There materials include langasite and ferroelectric ceramics with a perovskite structure, tungsten bronze structures, and bismuth layer-structured ferroelectrics (BLSF) [31, 47]. Recently, various perovskite-structured ferroelectrics based on $\mathrm{BaTiO}_{3}$ structure, such as $\left(\mathrm{Bi}_{1 / 2} \mathrm{Na}_{1 / 2}\right) \mathrm{TiO}_{3},\left(\mathrm{Bi}_{1 / 2} \mathrm{~K}_{1 / 2}\right) \mathrm{TiO}_{3}, \mathrm{KNbO}_{3}$, or $(\mathrm{K}, \mathrm{Na}) \mathrm{NbO}_{3}$ [31], and their solid solutions have been actively studied as candidates for new lead-free piezoelectric ceramics.

At the moment no lead-free materials displays better piezoelectric properties than those of PZT-based systems. To replace PZT-based systems, it is necessary that the required piezoelectric properties can be tuned for the specific application [47], the main issues including to improve their low Curie temperatures or depolarization temperatures, $T_{d}$, difficulties in poling treatments, and/or low relative densities.

Barium titanate $\left(\mathrm{BaTiO}_{3}\right)$ is a representative perovskite compound with an even extensive history than PZT with ferro- and piezoelectric properties and a large diversity of commercial applications for devices. The applications of $\mathrm{BaTiO}_{3}$ include ceramic capacitors, embedded capacitance in printed circuit boards, underwater transducers (sonars), thermistors with positive temperature coefficient of resistivity and electroluminescent panels [16], among others. Their grain size plays a major role in ferroelectric properties. This ceramic has a relatively high electromechanical coupling factor, $\mathrm{k}_{33} \approx 0.50$, and piezoelectric strain constant, $\mathrm{d}_{33} \approx 190 \mathrm{pC} / \mathrm{N}$, and has been used for piezoelectric applications such as sonars and dielectric material for multilayered ceramic capacitors due to its low Curie temperature and low coercive field. However, the working 
temperature range of the $\mathrm{BaTiO}_{3}$ is narrow for actual piezoelectric applications due to its low $T_{c} \approx 130{ }^{\circ} \mathrm{C}$ and the vector of spontaneous polarization changes its orientation with decreasing the temperature [31]. The dielectric and piezoelectric properties of the material are affected by its stoichiometry and microstructure, as well as by the dopants in the solid solution. Ceramic $\mathrm{BaTiO}_{3}$ with dopants such as $\mathrm{Pb}$ or $\mathrm{Ca}$ ions has been used to stabilize the tetragonal phase over a wider temperature range and has been used as a commercial piezoelectric material [26]. $\mathrm{BaTiO}_{3}$ has attracted continuous interest because of its simpler composition when compared to PZT and there are many scientific studies using $\mathrm{BaTiO}_{3}$ as a model material to investigate the nanoscale effects.

$\mathrm{BaTiO}_{3}$-based doped ceramic materials with sodium or potassium can be improve the piezoelectric properties, but their inherently low Curie temperature and several polymorphic phase transitions limits its overall usefulness. In this way, new ceramic composites were investigated to improve the overall properties of lead-free ceramics.

Bismuth sodium titanate $\left(\left(\mathrm{Bi}_{1 / 2} \mathrm{Na}_{1 / 2}\right) \mathrm{TiO}_{3}\right)$ or $\mathrm{BNT}$ is the most important compound among the bismuth containing ferroelectric perovskites. Their perovskite-structured is ferroelectric with Curie temperature between $340 \leq T_{c} \leq 540$ and exhibits a large remnant polarization, strong ferroelectric properties and relatively high piezoelectric response compared with other lead-free piezoelectric ceramics [50]. Thus, the piezoelectric constant is $d_{33} \approx 57$ to $64 \mathrm{pC} / \mathrm{N}$ for pure BNT. On the other hand, BNT ceramics need high temperatures for being processed and it is difficult to pole due to their high electrical conductivity. Other BNT-based structures with a morphotropic phase boundary are reported in order to improve the piezoelectric properties (as $d_{33}$ or $k_{33}$ ) and increase the Curie temperature $[31,48]$. Doping BNT ceramics with some inorganic materials improves the overall properties and processing when compared with pure BNT ceramics [48]. The piezoelectric charge constant can be larger than $d_{33}>100$ and the Curie temperature is near $350{ }^{\circ} \mathrm{C}$ when $\mathrm{BNT}$ is doped with niobium sodium oxide $\left(\mathrm{NaNbO}_{3}\right)$ [48]. There are other materials that have been used as dopants [42, 48], but do not significantly change the properties of these ceramic systems in view of commercial applications.

Replacing sodium by potassium leads to bismuth potassium titanate $\left(\left(\mathrm{Bi}_{1 / 2} \mathrm{~K}_{1 / 2}\right) \mathrm{TiO}_{3}\right)$, BKT, a lead-free ferroelectric with a perovskite structure of tetragonal symmetry at room temperature with relatively high Curie temperature, $T_{c}=380^{\circ} \mathrm{C}[31,51]$. Due to its promising properties, there are extensive research efforts in BKT-based solid solutions $[31,42]$. The combination ceramic systems such as $\mathrm{BKT}, \mathrm{BaTiO}_{3}$ and $\mathrm{BNT}$ has already 
been investigated, leading to overall properties similar to the individual properties of each compound.

The dielectric and piezoelectric properties of $(1-x) \mathrm{BT}-x \mathrm{BKT}$ solid solution leads to large piezoelectric constants, $d_{31}=350 \mathrm{pC} / \mathrm{N}$ and $d_{33}=450 \mathrm{pC} / \mathrm{N}$, and a relatively high $T_{c}=380$ ${ }^{\circ} \mathrm{C}$ were reported [31]. Others compounds based on $\mathrm{BaTiO}_{3}$ with specific properties are detailed in [48]. Thus, there are investigations in $\mathrm{BaTiO}_{3}$ ceramic doped with sodium, potassium and lithium to develop new ceramic systems, $\mathrm{Bi}(\mathrm{Na}, \mathrm{K}, \mathrm{Li}) \mathrm{TiO}_{3}$, but generally, the overall properties are comparable to barium titanate [48]

Zinc oxide $(\mathrm{ZnO})$ is another important lead-free piezoelectric. $\mathrm{ZnO}$ is highly tensile and may undergo large mechanical deformations, receiving increased attention for MEMS device applications. Due to the unique combinations of electrical, optical and piezoelectric properties, $\mathrm{ZnO}$, has a large potential for energy harvesting applications [5254], being demonstrated with this material an innovative approach for converting nanoscale mechanical energy into electric energy by piezoelectric zinc oxide nanowire (NW) arrays [54].

Another alternative material of the perovskite family of lead-free piezoceramics is potassium niobate $\mathrm{KNbO}_{3}(\mathrm{KN})$ and doped with sodium, the potassium-sodium niobate (K,Na) $\mathrm{NbO}_{3}(\mathrm{KNN})$. KN exhibits the same sequence of phase transitions as $\mathrm{BaTiO}_{3}$ with high Curie temperature, $T_{c}=435{ }^{\circ} \mathrm{C}$, but shows weak piezoelectric properties. The tetragonal, orthorhombic and rhombohedral phases are all ferroelectric in KN. Strong research efforts have been carried out in lead-free piezoelectric KNN-based ceramics in last decade leading to a large enhancement in piezoelectric charge coefficients from $d_{33} \approx 100$ to $\approx 500 \mathrm{pC} / \mathrm{N}[25,31,48,50]$. Further, relatively low dielectric constants and high electromechanical coupling factors could be obtained over a wide range of compositions. $\mathrm{KNN}$ is one of the most promising alternatives to $\mathrm{Pb}$-containing piezoelectric ceramics with high $T_{c} \approx 420{ }^{\circ} \mathrm{C}$, strong ferroelectricity [50] and good piezoelectric properties [31], although their properties are still inferior to PZT ceramic. New synthesis methods as nanocrystals (1D materials) can improve the KNN properties [25]. The introduction of $\mathrm{ABO}_{3}$-type compounds produces strong "softening" effects in $\mathrm{KNN}$-based ceramics, giving significant improvement in piezoelectric properties. KNNbased ceramics such as $\mathrm{K}_{0.5} \mathrm{Na}_{0.5} \mathrm{NbO}_{3}-\mathrm{K}_{5.4} \mathrm{Cu}_{1.3} \mathrm{Ta}_{10} \mathrm{O}_{29}-\mathrm{MnO}_{2}(\mathrm{KNN}-\mathrm{KCT}-\mathrm{Mn})$ or KNN-potassium cooper titanate-manganese ceramic have been prepared by conventional ceramic sintering technique, KNN-KCT-Mn showing excellent piezoelectric properties for application in power devices [25]. Different dopants can lead to different structures in 
piezoelectric KNN-based ceramics with interesting properties [31, 50]. Copper oxide on KNN ceramics decreases the synthesis temperature of $\mathrm{KNN}$, prevent the sodium oxide evaporation and eventually improves the piezoelectric properties by increasing the poling efficiency [48]. There are other investigations to improve the processing method or tailoring the properties of $\mathrm{KNN}$ ceramic, without strong improvements in the piezoelectric properties of the ceramics.

The main properties of the abovementioned materials are summarized in Table 6 .

Table 6: Main properties of the most representative piezoelectric ceramics suitable for energy harvesting applications.

\begin{tabular}{|c|c|c|c|c|c|c|}
\hline Materials & $\begin{array}{c}\text { PE charge } \\
\text { constant } \\
d_{33}(\mathrm{pC} / \mathrm{N})\end{array}$ & $\begin{array}{c}\text { PE charge } \\
\text { constant } \\
d_{3 I}(\mathrm{pC} / \mathrm{N})\end{array}$ & $\begin{array}{c}\text { EM } \\
\text { coupling } \\
\text { factor } k_{33}\end{array}$ & $\begin{array}{c}\text { Dielectric } \\
\text { constant } \\
\varepsilon_{r}\end{array}$ & $\begin{array}{c}\boldsymbol{T}_{\boldsymbol{c}} \\
\left({ }^{\circ} \mathbf{C}\right)\end{array}$ & Ref. \\
\hline PT & - & - & - & - & 490 & [41] \\
\hline PZT & 472 & - & 0.71 & 714 & 340 & {$[15,45]$} \\
\hline PZT4 & $290-410$ & 170 & 0.6 & 2300 & 250 & {$[37,42]$} \\
\hline PZT5A & 375 & - & 0.71 & 1700 & 365 & {$[42]$} \\
\hline PZT5H & 590 & - & 0.75 & 3400 & 190 & {$[42]$} \\
\hline PZT8 & 225 & - & 0.64 & 1000 & 300 & {$[42]$} \\
\hline PLZT & - & - & 0.45 & 1800 & - & {$[55]$} \\
\hline $\mathrm{BaTiO}_{3}$ & 190 & - & 0.5 & 1700 & 115 & {$[42,48]$} \\
\hline $\mathrm{ZnO}$ & 12.4 & 5.0 & 0.48 & 10.9 & - & {$[23,53,56]$} \\
\hline $\mathrm{KNN}$ & $>80$ & - & $<0.6$ & $<500$ & 421 & {$[42,50]$} \\
\hline KNN-KCT-Mn & 90 & -33 & 0.4 & 300 & 404 & [27] \\
\hline BNT & 64 & - & - & 302.6 & 310 & {$[31,42,48]$} \\
\hline BKT & 74 & - & - & - & 380 & {$[31,51]$} \\
\hline BNLT & $<91$ & - & - & $<550$ & $<370$ & {$[57]$} \\
\hline BNKLT & $41-145$ & - & - & $<1753$ & - & [58] \\
\hline BNT-NN & $<80$ & - & - & $<801$ & - & [59] \\
\hline BNT-BZT & $<147$ & - & - & $<881$ & $<251$ & {$[60]$} \\
\hline
\end{tabular}

Additional ceramic materials with piezoelectric properties are aurivillius and tungsten bronze ceramics or pyrochlore structures but due to their processability issues, such as property reproducibility difficulty, or intrinsic low properties are currently hardly to use in commercial devices.

The processing methods used in the fabrication of the ceramics materials span from powder synthesis, colloidal processing to template grain growth [16]. Generally, the several processing methods involve mechanical, thermal and chemical processes. The 
control of each processing step, purity and size control of the ceramic crystals is critical for the final overall properties of the ceramics materials. In the last decade, research on the controlled processing at nanoscale of this kind of materials has been extended to new compositions and routes to produce powders with controlled properties. Nano- and microfabrication improves the intrinsic properties of the ceramics. One-dimensional piezoelectric fabrication methods for lead and lead-free nanomaterials has become a research topic of interest in last year's due their exceptional morphology structure and excellent piezoelectric properties. The research of these kind of nanomaterials have been carried out in three different ways: controllable synthesis, emerging characterization techniques and novel fabrication techniques of nanomaterials [25]. Actually the piezoelectric nanostructures can be fabricated in several forms such as nanorods, nanowires or nanotubes and can be prepared either with random orientation or aligned using controllable synthesis. High resolution characterization techniques are also promoting the suitable understanding of the properties of these structures at micro- and nanoscale. Finally, novel fabrication methods, such as printing techniques, allow to develop new application areas

\subsection{Piezoelectric Polymers}

Kawai's pioneering works in the area of piezoelectric polymers in the late 60's of the last century led to strong research activity in poly(vinylidene fluoride) (PVDF) and its copolymers [61].

Electroactive polymers are among the most interesting classes of polymers used as smart materials in numerous applications including sensors, actuators, energy harvesting devices and biomedical applications [62].

The piezoelectric properties of polymers are related to the molecular structure of the polymer (monomer characteristics and chain conformation) and its orientation. The main disadvantage of piezoelectric polymers is that their piezoelectric coefficients $\left(d_{33} \leq 30\right.$ $\mathrm{pC} / \mathrm{N}$ ) are lower than for other piezoelectric materials [63]. However, their piezoelectric stress constant is much higher, which indicates that they are well suited for sensors. Other relevant advantages of polymers areas their relatively low-cost processing and light weight (low density) and high flexibility. Further, they show low dielectric constant and high dielectric breakdown.

Piezoelectric polymers can be divided into two main categories: semicrystalline (the majority of piezoelectric polymers) and amorphous polymers. Piezoelectric 
semicrystalline polymers show a polar crystalline phase. Mechanical orientation, thermal annealing or high voltage treatment are all effective to induce the piezoelectric crystalline phase within the polymer. The piezoelectric effect can be found in a non-crystalline polymer if its molecular structure contains strong molecular dipoles. Amorphous piezoelectric polymers, however, usually exhibit a lower piezoelectric response than semicrystalline polymers. Their corresponding polymer based piezoelectric composites are described in a separate section.

Both in semicrystalline and amorphous polymers, there are specific structural properties that should exist for a polymer material to show piezoelectric properties. First, the polymer molecular structure must inherently contain molecular dipoles. Second, these dipoles should be able to be reoriented within the material and to keep in their favored orientation state. This reorientation is achieved through the application of an electric field, a process called poling [23].

\subsubsection{Semicrystalline polymers}

Piezoelectric polymers with semicrystalline structure presenting piezo, pyro or ferroelectricity include Nylon-11, the family of the poly( $\alpha$-hydroxy acid)s, and PVDF and its copolymers.

PVDF was discovered in 1969 by Kawai and is the most widely reported piezoelectric polymer, being up to now the best all-around electroactive polymer and being therefore the polymer of choice for an increasing number of applications [62].

PVDF is a semicrystalline polymer and can present four distinct crystalline phases related to different chain conformations [62]. The crystalline properties of PVDF are strongly dependent on the processing conditions, which as stablished in order to get a specific phase or the coexistence of different polymorphs within the semicrystalline polymer. Figure 11 shows the phases most investigated and used for applications: $\alpha, \beta$ and $\gamma$ phases, among which the $\alpha$-phase is nonpolar and the $\beta$-phase is the most polar. The piezoelectric coefficients of poled thin films of the PVDF in the $\beta$ phase were reported to be as large as $-34 \mathrm{pC} / \mathrm{N}$, larger than the values observed in any other polymer [62]. PVDF has typically $50-60 \%$ of crystalline phase and a glass transition temperature $\left(T_{g}\right)$ around -35 ${ }^{\circ} \mathrm{C}$ [26]. To obtain the material in its piezoelectric phase, the $\alpha$ to $\beta$ phase transformation is typically achieved by mechanically stretching in order to orient the molecular chains and then poled at few hundred MV/m. It is to notice that the crystallization of PVDF in its $\beta$-phase can be also achieved using several strategies, including electrospinning 
processing [64], or in the presence of nanofillers such as $\mathrm{BaTiO}_{3}$ [65], organically modified clays [66], ionic liquids [67], or magnetic nanofillers [68], among others. Unlike other piezoelectric materials, PVDF has a negative $d_{33}$ value.

The preparation method can influence the overall polymer properties and, for example, PVDF electrospun fibers can be prepared in the $\beta$-phase with piezoelectric constants four times higher than those of corresponding thin films [69].

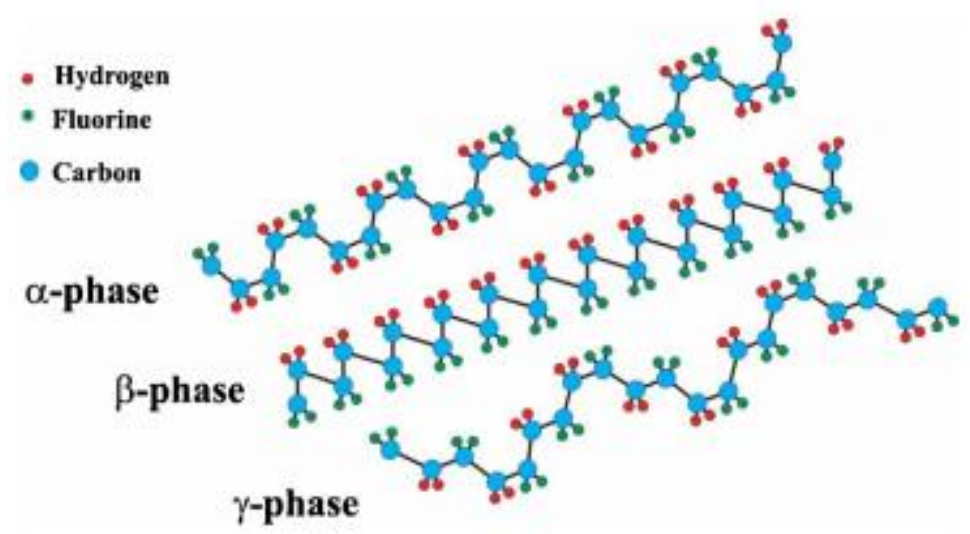

Figure 6: Schematic representation of the chain conformation for the $\alpha, \beta$ and $\gamma$ phases of PVDF [62].

PVDF based materials in the form of copolymers and terpolymers with distinct behaviors in response to electric fields [17] have been developed. The copolymer PVDF-TrFE is synthesized through free radical polymerization of the two monomers, an example of defect-modified PVDF. Copolymer molecular interchain interactions leads to a decrease of the ferroelectric to paraelectric transition temperature $\left(T_{C}\right)$ below the melting temperature of the polymer [17]. The transition temperature of the copolymer material depends on the molar ratio of TrFE, decreasing with increasing the molar ratio between 60 to $135^{\circ} \mathrm{C}$ [17]. PVDF and PVDF-TrFE show similar mechanical properties, as well as dielectric properties. On the other hand, the piezoelectric coefficient $\mathrm{d}_{33}$ and $\mathrm{k}_{33}$ are larger in the copolymer compared to pure PVDF [23]. The other piezoelectric coefficients are similar between both polymers. PVDF-TrFE has been thus used in sensor applications due to the better piezoelectric properties than PVDF [38].

Another PVDF copolymers developed using bulkier monomers such as chlorotrifluoroethylene (CTFE) and hexafluoropropylene (HFP), and more recently the bromotrifluoroethylene (BTFE) have been developed. PVDF-CTFE and PVDF-HFP exhibit slimmer electrical hysteresis loops when compared with related polymers based on PVDF. In PVDF and their copolymers the poling procedure is critical to obtain stable 
and high level piezoelectric response. Poling can be achieved by applying a DC field across the film thickness at a temperatures between 80 and $100{ }^{\circ} \mathrm{C}$ for a period around 30 min and should remain applied while the sample cools to room temperature to not depolarize the sample at higher temperatures. The poling fields to produce the maximum polarization are just below the breakdown strength, typically 30-120 MV/m. Corona poling is also typically used to pole PVDF and related copolymer samples.

Polyurea is a thermoset elastomer formed by the rapid reaction of isocyanates and amines, having microphase separation between hard and soft domains, with high and low $T_{g}$, respectively. The elastomer is composed by crystalline hard domains dispersed in the continuous soft segment matrix. Polyurea shows excellent mechanical and physical properties, impact mitigation, moisture and chemical resistance, long elongation and a wide range of operating temperatures. These properties makes it a suitable candidate for many engineering applications [70]. Aromatic and aliphatic (with an odd number of $\mathrm{CH}_{2}$ groups) polyureas are expected to have good ferroelectric properties because of the large dipole moment of the urea bond, which is more than twice that of $\mathrm{P}(\mathrm{VDF} / \mathrm{TrFE})$ [71].

Aromatic polyureas were the first polyurea structures identified as piezoelectric, showing piezoelectric properties up to high temperatures, near $200^{\circ} \mathrm{C}$ [72].

Odd-numbered nylons (polyamides polymers) are one of the most investigated semicrystalline polymers, presenting excellent piezoelectric properties at elevated temperatures. The monomer unit of odd nylons (usually nylon-5, nylon-7 and nylon-11) consists of even numbers of methylene groups and one amide group with a dipole moment [72]. The amide dipoles align synergistically for the odd-numbered monomer, resulting in a net dipole moment.

Biopolymers are an interesting area of study in the scope of piezoelectric polymer due the wide range of potential biomedical application. The piezoelectricity has been first observed in 1941 and studied in several biopolymers that present lower piezoelectric constant than synthetic polymers. The electromechanical effect in biopolymers is attributed to the internal rotation of polar atomic groups linked to asymmetrical carbon atoms. These biopolymers include collagen, polypeptides like poly $(\gamma$-methylglutamate) and poly( $\gamma$-benzyl-L-glutamate), oriented films of DNA, poly( $\alpha$-hydroxy acid)s family and chitin. Biopolymers from natural origin are often difficult to process and show poor mechanical and electrical properties [73]. In this scope, several synthetic biopolymers have been widely used to produce materials for tissue engineering [73]. The poly $(\alpha-$ hydroxy acid)s family such as polyglycolide (PGA), polylactide (PLA) and its copolymer 
like poly(lactide-coglycolide) (PLGA) which are synthetic polymers approved for human clinical use by FDA are extensively used or tested for scaffolds materials as a bioerodible material due to good biocompatibility, controllable biodegradabilitiy, and relatively good processability [74].

\subsubsection{Amorphous polymers}

The literature on semicrystalline piezoelectric polymers literature is more extensive than for amorphous polymers. This is in part due to the lower piezoelectric response of amorphous piezoelectric polymers, which are not enough to attract commercial interest. The piezoelectric effect in amorphous polymers diverges from that in semicrystalline ones, once the polarization is rather a quasi-stable state due to the freezing-in of molecular dipoles and is not in a state of thermal equilibrium.

The orientation polarization of molecular dipoles is responsible for the piezoelectricity in amorphous polymers. The polymers are polarized by an external field above their glass transition, where the mobility of the molecular chains is larger and allow the dipole alignment with the electric field. The remaining polarization is achieved below $T_{g}$, resulting in a piezoelectric-like effect proportional to the electric field of polarization [75]. Examples of the amorphous piezoelectric polymers include polyamide and nitrilesubstituted polymers, including polyacrylonitrile (PAN), poly(vinylidene cyanide-vinyl acetate) (PVDCN/VAc), poly(phenyl ether nitrile) (PPEN) and poly(1biciclobutanecarbonitrile). Weak piezoelectric properties in polyvinyl chloride (PVC) and polyvinyl acetate (PVAc) have also been found.

The most interesting of these materials are vinylidene cyanide and vinyl acetate copolymer, which have been extensively studied due to their suitable piezoelectric response and large dielectric relaxation strengths.

Poly(vinylidene chloride) (PVDC) shows lower piezoelectric properties but is stable and reproducible, improving by simultaneous stretching and corona poling of the film [75]. Poly(vinylidene chloride nitrile), PVDCN/VAc, shows large relaxation strength and has the largest value of remanent polarization and piezoelectric stress effect of all amorphous polymers [75]. Polyacrylonitrile (PAN) is a widely used polymer and has similar structure than PVDCN/VAc. PAN has large nitrile dipoles that can be oriented by an extern electric field.

Polyimide $(\beta-\mathrm{CN}) \mathrm{APB} / \mathrm{ODPA}$ shows piezoelectric properties but, however, one order of magnitude smaller than PVDF. These lower piezoelectric properties are in part due to the 
fact that the dipoles in the polymer do not align along the applied electric field efficiently because of limited chain mobility in the imidized closed ring structure [76]. The high temperature performance of these modified polyimide films allow to maintain their piezoelectric properties at temperatures up to $150{ }^{\circ} \mathrm{C}$ [77]. Therefore, thermally stable piezoelectric polyimides may be an attractive alternative to PVDF for high temperature piezoelectric applications [76].

The main relevant properties of the aforementioned piezoelectric polymers are summarized in Table 7.

Table 7: Main properties of the presented piezoelectric polymers (semicrystalline and amorphous) potentially interesting for energy harvesting applications.

\begin{tabular}{|c|c|c|c|c|c|}
\hline Materials & $\begin{array}{c}\text { PE charge } \\
\text { constant } \\
d_{31}(p C / N)\end{array}$ & $\begin{array}{c}\text { EM coupling } \\
\text { factor }_{31}\end{array}$ & $\begin{array}{c}\text { Dielectric } \\
\text { constant } \varepsilon_{r}\end{array}$ & $\begin{array}{c}\boldsymbol{T}_{g} \\
\left({ }^{\circ} \mathbf{C}\right)\end{array}$ & Ref. \\
\hline PVDF & $6-34$ & 0.12 & 12 & -35 & {$[23,61,62]$} \\
\hline PVDF/TrFE & $6-12$ & 0.07 & 12 & -35 & [23] \\
\hline Polyurea & $<10$ & - & - & - & {$[72,78]$} \\
\hline Nylon-7 & 17 & 0.054 & & - & [61] \\
\hline Nylon-11 & 14 & 0.049 & & 50 & [72] \\
\hline PAN & 2 & 0.01 & 38 & 90 & [61] \\
\hline PVDCN/VAc & 10 & 0.05 & 3 & 170 & {$[61,72,79]$} \\
\hline PPEN & - & - & 5 & 145 & [80] \\
\hline PVC & $0.5-5$ & 0.001 & 10 & 80 & {$[61,81]$} \\
\hline PVAc & - & - & 6.5 & 30 & [61] \\
\hline Polyimide & $<5$ & - & 4 & 220 & {$[23,61,76,77]$} \\
\hline
\end{tabular}

\subsection{Piezoelectric Polymer Based Composites}

Piezoelectric composites are typically composed by a piezoelectric ceramic or crystalline phase embedded in a polymer matrix. The polymer gives the mechanical properties as flexibility or pliability, but it can be also piezoelectric, such as PVDF.

Piezoelectric composites are usually defined by their dimensional connectivity, represented in Figure 12. The connectivity between polymer and ceramic or crystal is a critical parameter in a composite material. A composite with m-n connectivity has its piezoelectric phase connected in $\mathrm{m}$ dimensions for the polymer phase and in $\mathrm{n}$ dimensions 
for ceramics or crystals. For example, 0-3 structure comprises dispersed piezoelectric particles in a polymer matrix and 1-3 consist of cylindrical or square ceramic or crystal pillars embedded in a polymer matrix (Figure 12). There are different methods to fabricate a 1-3 composite. The two most common methods are dice-and-fill and laminate-and-cut [59]. The 0-3 composite fabrication is the most simple, just by particles dispersion on a polymer. These two configurations are the most used in composites materials.

The main goal of piezocomposite structures is to combine the best properties of the polymer matrices (mechanical, electrical or piezoelectric) with the excellent piezoelectric properties of the ceramics or single crystals. However, there are some disadvantages, such as the operation temperature of the device, which is typically lower when compared to ceramic or crystal properties, piezoelectric losses in particle-polymer interfaces and viable processing during the manufacture of the device. Particles size, dispersion in polymer, manufacturing using solvents and homogenization of the overall properties in the composite are challenges which are still investigated nowadays.

Piezoelectric composite materials also offer the possibility of increased customization through the selection of the constituent material phases and filler volume fraction. Their combination changes the final properties of the composite material that can be tailored for specific applications.

Piezoelectric composite materials increase the performance of ultrasonic transducers particularly in applications where there is a large acoustic impedance mismatch between the piezoelectric ceramic and the ultrasound propagation medium.
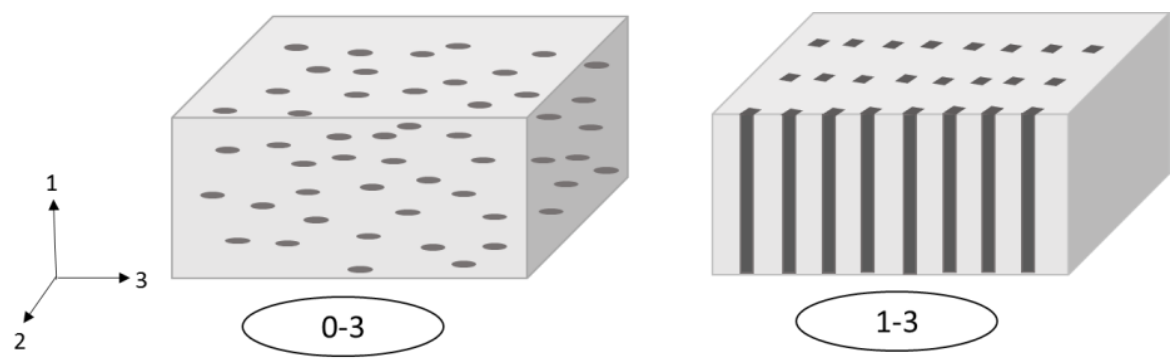

Figure 7: Piezocomposite material structures with 0-3 and 1-3 connectivity. Polymer is shown as uniform grey, ceramic textured [26].

The typical examples of ceramics used in composites are $\mathrm{BaTiO}_{3}$, PZT and PMN-PT. Elastomers or thermoplastic elastomers are commonly used in piezoelectric composites due their mechanical properties, including high flexibility or deformability. 
Thermoplastics are used in distinct applications with bending or small applied deformations.

Summarizing, the different materials with piezoelectric properties range from natural materials such as wood and silk to biological ones, such as bone and tendon, among others. The most relevant natural piezoelectric crystals are quartz, Rochelle salt and tourmaline. Man-made piezoelectric materials include single crystal, ceramics and polymer, being the most relevant $\mathrm{BaTiO}_{3}$ and $\mathrm{PZT}$ among the ceramic materials and PVDF as the polymer one. In the last decades lead-free regulations have fostered the study and development of new high-performance lead-free ceramic materials.

\section{Results and Discussion}

This section will provide an overview of piezoelectric energy harvesting applications divided into four distinct parts according to the nature of the material used in the device: ceramics, single crystals, polymers and polymer composites. The order of presentation followed in this chapter is related with the number of works reported for each type of material. In each subsection it is presented a table with the materials used in the application, the performance and the type of device produced. This section is not an exhaustive review of all available literature, but rather a representative summary of the main applications with the most used materials and geometries.

\subsection{Piezoelectric energy harvesting based on ceramics}

Piezoelectric ceramics are widely used in energy harvesting systems because of their high energy conversion ability, large electro-mechanical coupling constants, ease of incorporation into devices and low cost. The typical piezoelectric ceramics are $\mathrm{BaTiO}_{3}$, $\mathrm{ZnO}$ and PZT, the later stands out due to its excellent piezoelectric properties, high dielectric constants and high Curie temperature, resulting in a wide number of studies and applications. The wide applicability of PZT boosted the expansion of this material to a large family of ceramics by chemical composition modifications, among which highlights PZT-5H and PZT-5A as being the most often used [34, 82-84]. The potential of these materials has been extensively explored by the scientific community in seeking solutions for a better use of the mechanical energy available in the environment and thus, several studies have been devoted to the development of piezoelectric energy harvesters based 
on piezoceramics. Table 8 summarizes the power outputs and main characteristics of several energy harvesting systems based on piezoceramics reported in the literature.

Table 8: Summary of the piezoelectric energy harvesting systems based on ceramics.

\begin{tabular}{|c|c|c|c|}
\hline $\begin{array}{l}\text { Piezoelectric } \\
\text { material }\end{array}$ & $\begin{array}{c}\text { Output } \\
\text { Performance }\end{array}$ & Characteristics & Ref. \\
\hline \multirow{14}{*}{ PZT } & $3.85 \mathrm{~mW}$ & \multirow{21}{*}{ Cantilever beam } & {$[85]$} \\
\hline & $2.9 \mathrm{~mW}$ & & [86] \\
\hline & $5.5 \mu \mathrm{W}$ & & {$[87]$} \\
\hline & $1.38 \mathrm{~mW}$ & & {$[88]$} \\
\hline & $2.4 \mu \mathrm{W}$ & & [89] \\
\hline & $2.15 \mu \mathrm{W}$ & & [90] \\
\hline & $0.803 \mu \mathrm{W}$ & & [90] \\
\hline & $4.5 \mu \mathrm{W}$ & & {$[91]$} \\
\hline & $51.4 \mu \mathrm{W}$ & & {$[92]$} \\
\hline & $1.4 \mu \mathrm{W}$ & & {$[93]$} \\
\hline & $2.16 \mu \mathrm{W}$ & & {$[94]$} \\
\hline & $1.01 \mu \mathrm{W}$ & & {$[95]$} \\
\hline & $0.66 \mathrm{~mW}$ & & {$[96]$} \\
\hline & $70 \mu \mathrm{W}$ & & [97] \\
\hline \multirow{2}{*}{ PZT-5A } & $\sim 8 \mathrm{~mW}$ & & [98] \\
\hline & $375 \mu \mathrm{W}$ & & {$[99]$} \\
\hline PZT-5H & $0.48 \mathrm{~mW}$ & & [100] \\
\hline PZT-5A4E & $4.05 \mu \mathrm{W} / \mathrm{mm}^{3}$ & & {$[101]$} \\
\hline PZT (PSI-5A4E) & $38.1 \mu \mathrm{W}$ & & [102] \\
\hline PZT (PZT-QA) & $0.14 \mathrm{~mW}$ & & [103] \\
\hline $\mathrm{ZnO}$ & $\sim 20 \mu \mathrm{W}$ & & {$[104]$} \\
\hline $\mathrm{PZT}$ & $1.8 \mathrm{~W}$ & \multirow{3}{*}{ Rodway energy harvester } & [105] \\
\hline PZT-5A & $1.0 \mathrm{~W}$ & & [105] \\
\hline PZT (PZT-PZNM) & $184 \mu \mathrm{W}$ & & [106] \\
\hline \multirow{2}{*}{$\mathrm{PZT}$} & $39 \mathrm{~mW}$ & \multirow{3}{*}{ Cymbal transducer } & [107] \\
\hline & $52 \mathrm{~mW}$ & & [108] \\
\hline PZT-5H & $141.61 \mu \mathrm{W}$ & & {$[109]$} \\
\hline PZT & $10.2 \mu \mathrm{W}$ & \multirow{2}{*}{ Micro-fabricated power generator } & [110] \\
\hline PZT-5A & $205 \mu \mathrm{W}$ & & [111] \\
\hline \multirow{8}{*}{ PZT } & $3.7 \mathrm{~mW}$ & Skin for vibrating surfaces & {$[112]$} \\
\hline & $0.6 \mathrm{~mW}$ & Harvester prototype for human motion & [113] \\
\hline & $10-80 \mu \mathrm{W}$ & Piezoelectric-magnetostrictive generator & [114] \\
\hline & $1.8 \mathrm{~mW}$ & Membrane transducer & [115] \\
\hline & $1.5 \mu \mathrm{W}$ & Rolling-based vibration harvester & [116] \\
\hline & $80 \mathrm{~mW}$ & Strip for shoes soles & {$[117]$} \\
\hline & $4.8 \mathrm{~mW}$ & Piezoelements embedded on orthopedic implants & {$[118]$} \\
\hline & $1.13 \mu \mathrm{W}$ & Radioisotope micropower generator (cantilever) & [119] \\
\hline \multirow{2}{*}{ PZT-5H } & $9.30 \mathrm{~mW}$ & Flexible patch on a closed chamber & [120] \\
\hline & $3.0 \mu \mathrm{W}$ & Thick-film inertial generator & [121] \\
\hline \multirow{2}{*}{ PZT-5A } & $\sim 0.75 \mathrm{~mW}$ & $\begin{array}{l}\text { Unidirectionally aligned fibers embedded in a resin } \\
\text { matrix }\end{array}$ & {$[122]$} \\
\hline & $300-400 \mu \mathrm{W}$ & $\begin{array}{l}\text { Tunable-resonance vibration energy scavenger } \\
\text { generator }\end{array}$ & [123] \\
\hline \multirow{2}{*}{$\mathrm{BaTiO}_{3}$} & $\sim 125.5 \mathrm{pW}$ & \multirow{2}{*}{ Nanowires based generator } & [124] \\
\hline & $\sim 123 \mathrm{pW}$ & & {$[125]$} \\
\hline
\end{tabular}




\begin{tabular}{|l|c|c|c|}
\hline & $0.17-0.20 \mathrm{~V}$ & \multirow{2}{*}[126]{} \\
\hline \multirow{5}{*}{$\mathrm{ZnO}$} & $397 \mathrm{~mW} / \mathrm{cm}^{2}$ & {$[127]$} \\
\cline { 2 - 2 } & $70 \mathrm{pW}$ & {$[49]$} \\
\cline { 2 - 2 } & $\sim 15 \mathrm{nW}$ & & {$[128]$} \\
\cline { 2 - 2 } & $\sim 8 \mathrm{pW}$ & & {$[124]$} \\
\cline { 2 - 2 } & $2.39 \mathrm{pW}$ & & {$[129]$} \\
\cline { 2 - 2 } & $0.123 \mu \mathrm{W}$ & & {$[130]$} \\
\hline $\mathrm{KNN}$ & $\sim 30 \mu \mathrm{W}$ & Nanorods based generator & {$[131]$} \\
\hline
\end{tabular}

Most of the developed piezoelectric harvesting systems are based on the cantilever beam configuration, either for macro devices or for MEMS applications, as they are simple to fabricate, allow large mechanical strains and mainly possesses a resonance frequency of the flexural modes significantly lower than other vibrational modes of a piezoelectric. These systems are usually composed by a thin piezoelectric layer bonded to a nonpiezoelectric one, frequently a metal, serving as a conductor of the generated charge, with the end fixed to take advantage of the flexural mode of the structure. The majority of the cantilevers present one (unimorph) or two (bimorph) active piezoelectric layers, nonetheless the bimorph configuration is the most widely used since it doubles the energy output of the harvester without significant increase in volume [34].

It was verified that the power output of a cantilever is proportional to the proof mass, so it should contain the largest proof mass that is possible within the space constraints [97] (Figure 13a). For a given piezoelectric material, the piezoelectric coefficient $d_{31}$ is typically lower than $d_{33}$, in this sense and in order to take advantage of the higher piezoelectric vibration mode $\left(\mathrm{d}_{33}\right)$ an interdigitated electrode design is used to obtain higher energy outputs (Figure 13b).

a)

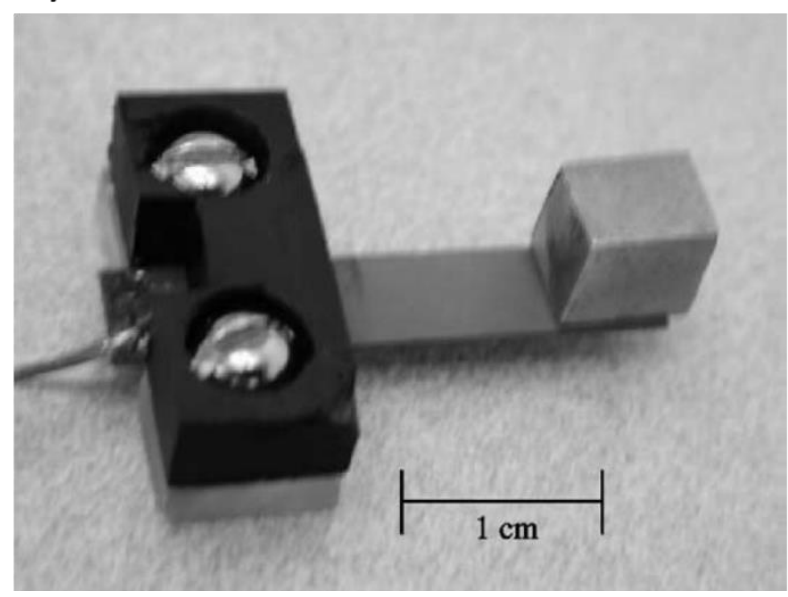

b)

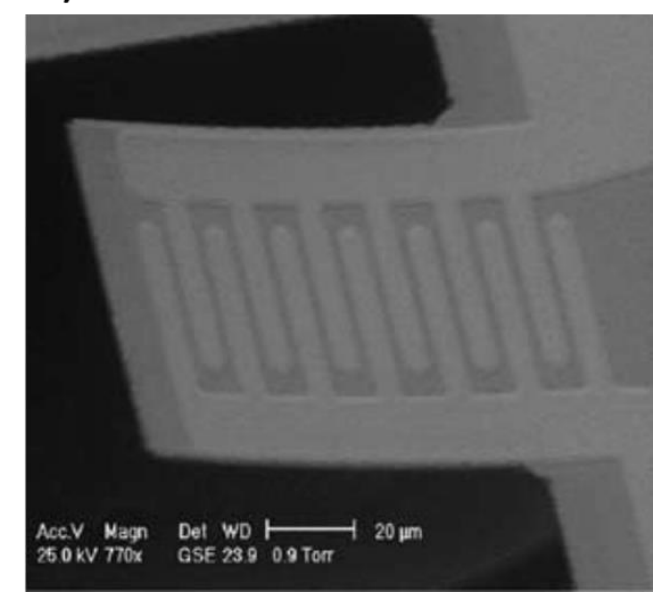

Figure 8: a) PZT cantilever beam with a proof mass attached [97] and b) SEM image of a cantilever beam with patterned interdigitated electrodes configuration [95]. 
The majority of these systems achieve output powers of some $\mu \mathrm{W}$, however it is possible to find reports in the order of $\mathrm{mW}$, as the rectangular bimorph cantilever beam fabricated from PZT, with the dimensions of $60 \times 20 \times 0.70 \mathrm{~mm}$, tested in environments from room temperature up to $82^{\circ} \mathrm{C}$. A maximum output power of $1.38 \mathrm{~mW}$ was recorded with a tip mass of $1.32 \mathrm{~g}$ and a load resistance of $19.75 \mathrm{k} \Omega$, at room temperature. At higher temperatures $\left(82^{\circ} \mathrm{C}\right)$ the output decreased up to $0.74 \mathrm{~mW}$ [88]. The geometric profile of the cantilever beam influences the performance of the harvester and, in this sense, a comparative study between three different beam profiles has been carried out: linear, parabolic and cubic. The output of the cubic cantilever proved to be the most effective configuration, leading to a power output of $2.9 \mathrm{~mW}$ with an optimum resistance of $170.902 \mathrm{k} \Omega$ [86]. The typical cantilever beam represented in the Figure 13a, composed by a bimorph based of PZT with $\sim 1 \mathrm{~cm}^{3}$ and a proof mass attached to the free end generated a power density of $70 \mu \mathrm{W} / \mathrm{cm}^{3}$. Simulations indicate that further optimization of the design of the piezoelectric harvester would allow to achieve $250 \mu \mathrm{W} / \mathrm{cm}^{3}$ from a vibration source with an acceleration of $2.5 \mathrm{~m} / \mathrm{s}^{2}$ [97].

The integration of cantilever beams into MEMS energy harvesting devices has been studied as a viable alternative to power micro devices. A MEMS-based power generator, based on a sol-gel-spin-coated PZT thin film with $170 \times 260 \mu \mathrm{m}$, registered a maximum output power of $1.01 \mu \mathrm{W}$ delivered to a $5.2 \mathrm{M} \Omega$ load, corresponding to an energy density of $0.74 \mathrm{~mW} \mathrm{~h} / \mathrm{cm}^{2}$. This cantilever operated in the $\mathrm{d}_{33}$ mode and used interdigitated shape electrode deposited via e-beam evaporation and patterned using a lift-off procedure (Figure 13b) [95]. A thin PZT harvester of $1 \mu \mathrm{m}$ was produced by pulsed laser deposition technique reported a maximum power output power of $51.4 \mu \mathrm{W}$ with a sensitivity of 63.6 $\mu \mathrm{W} / \mathrm{g}^{2}$, for a system dimensions of $1.1 \times 5.0 \times 5.0 \mathrm{~mm}$ and a proof mass of $0.91 \mathrm{~g}$. The potential of this device for energy harvesting in car tires have been assessed by applying shock input excitation and a power output of a few $\mu \mathrm{W}$ 's has been obtained with accelerations in the order of $100 \mathrm{~g}$, which are sufficient to power sensor nodes in car tires [92].

Regarding the PZT chemical modified materials, some solutions employing PZT-5A have been explored. A piezoelectric energy harvester based on PZT-5A was designed to generate power using wind energy. The system composed by a bimorph piezoelectric cantilever beam, with $72 \times 0.267 \times 36 \mathrm{~mm}$ and tip mass of $0.024 \mathrm{~g}$, generated a power of $\sim 8 \mathrm{~mW}$ with an optimum load of $105 \mathrm{k} \Omega$ [98]. In the same line, PZT-5A4E has been 
tested in rectangular and triangular geometry beams composed by a layer of brass sandwiched between two layers of the piezoceramic. A maximum e power of $0.23 \mathrm{~mW}$ at $45.5 \mathrm{~Hz}$ was achieved for the triangular shape with an optimum resistance of $71 \mathrm{k} \Omega$, corresponding to a normalized power density of $4.05 \mu \mathrm{W} / \mathrm{mm}^{3}$, approximately $85 \%$ power density improvement with respecting to the rectangular beam, which was attributed to the larger average strain in the beam [101]. Different shape beam structures have been also compared for the piezoceramic PZT-5H, including rectangular, trapezoidal and truncated triangular energy harvesters with the same length $(20 \mathrm{~mm})$, root width $(25 \mathrm{~mm})$ and thickness $(0.2 \mathrm{~mm})$. The three beams were tested in experiments with acceleration of $2 \mathrm{~m} / \mathrm{s}^{2}$ and a proof mass of $5.7 \mathrm{~g}$. The truncated triangular cantilever reached the highest open circuit voltage and optimal load power output of $18.25 \mathrm{~V}$ and $0.48 \mathrm{~mW}$, which was 81.6 and $167 \%$ higher than the $10.05 \mathrm{~V}$ and $0.18 \mathrm{~mW}$ obtained for the rectangular beam. The main reason for that difference was related with the strain distribution of the truncated triangular beam, which was more uniform than that of the rectangular and trapezoidal ones [100]. Further, piezoelectric PSI-5H4E with dimensions of $31.8 \times 12.7 \times 0.51 \mathrm{~mm}$ in a bimorph type with a center shim of brass, generated $38.1 \mu \mathrm{W}$ by using a resistance of $110.3 \mathrm{k} \Omega$ at the frequency range of $50 \pm 3 \mathrm{~Hz}$ and with a $4 \mathrm{~g}$ tip mass attached on the free end of the beam [102].

The concept of piezoelectric energy harvesters for busy roadways exploiting the vibrational frequency under vehicles speeds around 60-80 km/h have been explored. An energy harvester module, with $15 \times 15 \times 10 \mathrm{~cm}$, containing 12 cantilevers beams, with $38 \times 38 \times 0.2 \mathrm{~mm}$, based on commercial PZT-PZNM were produced as represented in Figure 14. Assuming that the most common speed of passage of the vehicles is between 60 and $80 \mathrm{~km} / \mathrm{h}$, corresponding to a road vibration between 10 and $14 \mathrm{~Hz}$, the frequency of the cantilever beams was adjusted for $13 \mathrm{~Hz}$. In these conditions, each module generated a power of $184 \mu \mathrm{W}$ with a power density of $8.19 \mathrm{~mW} / \mathrm{m}^{2}$, thus a full-scale harvesting module of $30 \times 30 \times 10 \mathrm{~cm}$ is expected to reach the $736 \mu \mathrm{W}$. In a place with a frequency of passage of 600 vehicles per hour each module can generate up to 4.91 $\mathrm{Wh} / \mathrm{m}^{2}[106]$. 


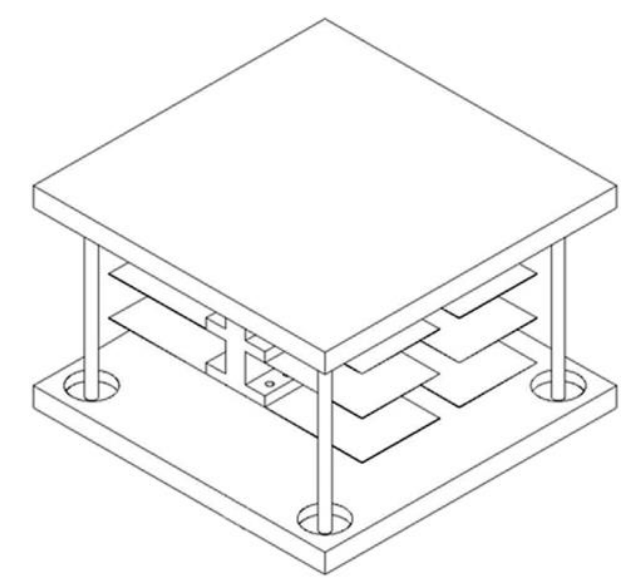

Figure 9: Three-dimensional schematic representation of a piezoelectric energy harvesting module $(15 \times$ $15 \times 10 \mathrm{~cm}$ ) to collect ambient energy from vehicles on a road [106].

Another approach for energy harvesters has been explored by varying materials and configurations. The better outputs were obtained for a prototype consisting of a stack of six ceramic disks of PZT-5A with $43 \mathrm{~mm}$ of diameter, $6.2 \mathrm{~mm}$ of height and a cross sectional area of $0.00176 \mathrm{~m}^{2}$, and for a set of 3 prototypes connected in parallel consisting of 21 rectangular PZT elements with $20 \times 20 \mathrm{~mm}$ in cross section and $2 \mathrm{~mm}$ in height. In both prototypes the elements were connected in parallel and their polarity alternated. The tests were performed using a servohydraulic universal testing machine with sinusoidal loading at $10 \mathrm{~Hz}$ with the mean of $2.5 \mathrm{kN}$ and amplitude of $2.5 \mathrm{kN}$. The prototype based on the PZT-5A ceramic disks generated $\sim 1.0 \mathrm{~W}$ while the porotype based on the PZT rectangular elements generated an output of $\sim 1.8 \mathrm{~W}$. The authors estimated the levelized cost of energy ranging from $\$ 19 / \mathrm{kWh}$ to $\$ 4.8 / \mathrm{kWh}$ for an average daily traffic ranging from 20000 to 80000 , and conclude that the piezoelectric energy harvesting from the roadways is attractive in powering equipment or electric lights in remote areas, since it is at least one order of magnitude more expensive than photovoltaics [105].

Cymbal transducers represent another harvester configuration frequently used to develop piezoelectric energy harvesting systems (Figure 15). Typically, it consists on a piezoelectric ceramic disc sandwiched between two cymbal-shaped metal end caps on each side, bonded directly to the surface of the disc using a suitable adhesive agent. When axial stress is applied to the cymbal transducer the end cap converts and amplify the axial stress to radial stress in the piezoelectric disc, such that both $d_{31}$ and $d_{33}$ piezoelectric coefficients are combined to contribute to the performance of the transducer. Cymbal transducers can provide higher energy output than cantilever beams because they withstand higher impact, however its robust nature limits the use to applications that 
provide high magnitude of vibrations, hence they are suitable for energy harvesting from natural ambient vibration sources which have low magnitude of vibrations [34, 132].

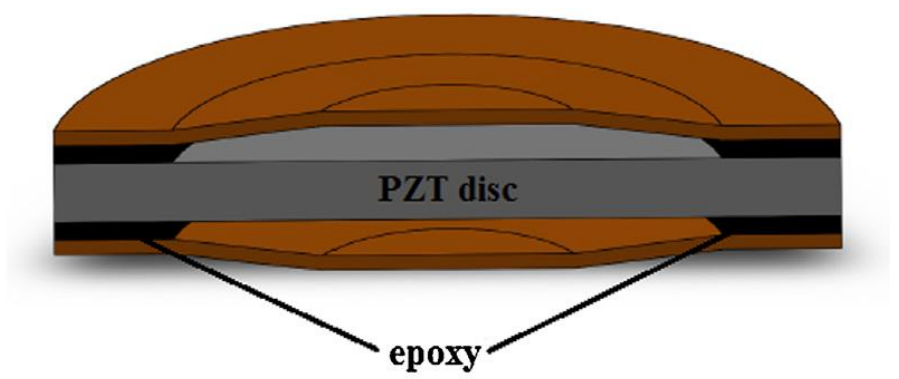

Figure 10: Schematic representation of a typical cymbal transducer [132].

A cymbal transducer, consisting of a piezoelectric ceramic disc sandwiched between two concave-shaped metal end-caps was used to test PZT discs of $29 \mathrm{~mm}$ diameter and $1 \mathrm{~mm}$ of thickness. The system generated a power of $39 \mathrm{~mW}$ under a cyclic force of $7.8 \mathrm{~N}$ at $100 \mathrm{~Hz}$ [107]. A power of $52 \mathrm{~mW}$ was measured across a $400 \mathrm{k} \Omega$ resistor in a cymbal transducer with a PZT ceramic of $29 \mathrm{~mm}$ diameter and an optimized thickness of $1.8 \mathrm{~mm}$ with an end cap of $0.4 \mathrm{~mm}$, under a force of $70 \mathrm{~N}$ and with a pre-stress load of $67 \mathrm{~N}$ at $100 \mathrm{~Hz}$. Applying higher stresses will allow to harvest power with higher efficiency, however the force magnitude of $70 \mathrm{~N}$ is near the maximum limit for a steel cap of $0.4 \mathrm{~mm}$ of thickness, thus the possibility to apply higher stresses is by increasing the diameter of the cymbal transducer in a specific ratio that allows using thicker end caps and ceramics [108]. The modified PZT-5H has also been employed in a cymbal transducer system consisting of a steel cantilever beam structure with a proof mass attached and two identical ceramic cymbal transducers as showed in Figure 16. The two tested systems consisted of a single layer bulk disk ceramic of $1 \mathrm{~mm}$ of thickness and a two layered stack disk ceramics with $0.5 \mathrm{~mm}$ of thickness. The stacked system generated more power than the single transducer, achieving $141.6 \mu \mathrm{W}$ at $153 \mathrm{~Hz}$ across a resistive load of $40 \mathrm{k} \Omega$, whereas the single one generated $104.04 \mu \mathrm{W}$ at $166 \mathrm{~Hz}$ with a load resistance of $80 \mathrm{k} \Omega$ [109]. 


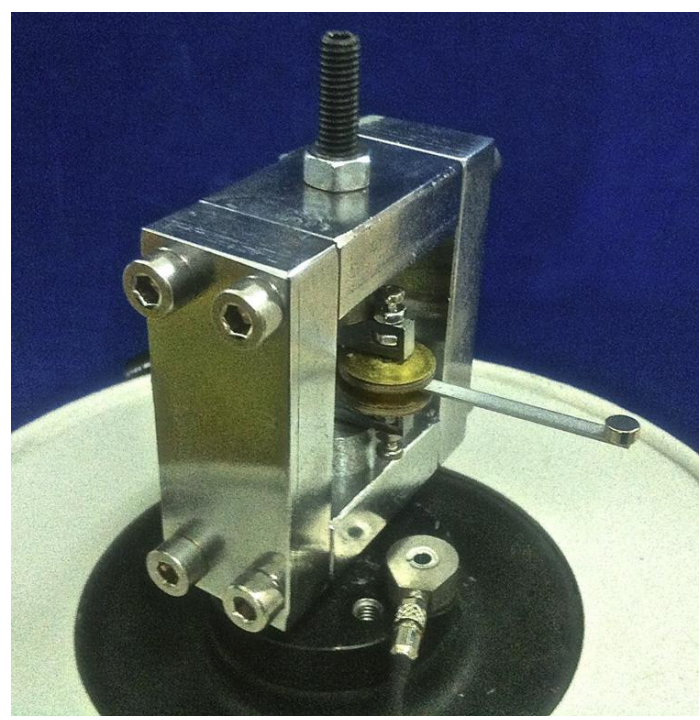

Figure 11: Piezoelectric energy harvester with two ceramic cymbal transducers and a steel cantilever beam with a proof mass attached [109].

An energy harvesting device achieves the maximum power output when the frequency of the external excitation matches the resonance frequency of the device [133]. In this sense the steel cantilever beam structure was used since it allows low resonance frequencies. Another configurations were studied and interesting outputs were achieved, such as the energy harvesting skin to attach onto vibrating surfaces developed based on PZT. The design considered two steps: the topology optimization to distribute the piezoelectric material for an effective energy harvesting and the shape optimization to ensure the manufacturability of the design model. The skin generated a power of $3.7 \mathrm{~mW}$ [112]. An unconventional approach using a rolling mechanism to convert vibrations into regular unidirectional rolling motion was tested. The system utilized an array of balls rolling the piezoelectric units of a PZT layer and achieved an output power of $\sim 1.5 \mu \mathrm{W}$ with a resistive load of 3.3 M $\Omega$, while the maximal rolling force was about $6.5 \mathrm{~N}$. This configuration presented the advantage of harvesting more vibration energy and reducing the impact forces caused by oscillation [116]. An unimorph piezoelectric membrane transducer composed by two layers of PZT/brass, perfectly embedded by an epoxy adhesive and with thickness of 230 and $400 \mu \mathrm{m}$ for PZT and brass layers respectively, achieved the maximum power output of $1.8 \mathrm{~mW}$ at the resonance frequency of $2.58 \mathrm{kHz}$, across an optimal resistor of $56 \mathrm{k} \Omega$ and under an acceleration of $2 g$ [115]. A patch configuration on a closed chamber that extracts energy from a hyperbaric air load was also investigated. The harvester prototype was composed by a flexible PZT-5H patch and was tested under different experimental conditions of pressure, load resistance, flow and 
cycle time. The results showed an optimal output power of $3.41 \mathrm{~mW}$ across $200 \mathrm{k} \Omega$ resistor, under $0.4 \mathrm{MPa}$ and $365 \mathrm{~L} / \mathrm{min}$ of pressure and flow respectively, during $0.8 \mathrm{~s}$ cycle [120].

The concept of energy harvesting from human motion has gained prominence in last years and several approaches were investigated for low power devices. In vivo electrical energy generation trough orthopedic implants were developed in total knee replacement implants. Three PZT elements, with a total volume of $1.2 \mathrm{~cm}^{3}$, were embedded within the implant and generated $4.8 \mathrm{~mW}$ of raw electrical power under the expected axial loading conditions corresponding to an efficiency of around $19 \%$. The raw PZT output was transformed into regulated electrical power through a conditioning circuit and an overall efficiency of $4 \%$ was achieved. The degradation study revealed a deterioration of less than $17 \%$ over the expected 20 -year lifetime of the implant. The development of more efficient conditioning circuitry will be considered in the future for further improvement of the system [118]. The biocompatibility of the energy harvester device is essential to develop in vivo applications, in this sense the search for piezoelectric materials of low toxicity, in particular lead-free ceramics, is of growing interest. Thus, a flexible lead free piezoelectric energy harvester based on $\mathrm{LiNbO}_{3}$-doped $(\mathrm{K}, \mathrm{Na}) \mathrm{NbO}_{3}(\mathrm{KNM})$ was produced by aerosol deposition and laser lift-off processes. The flexible piezoelectric energy harvester was tested by regular bending and random finger flicks generating 130 $\mathrm{V} / \sim 1.3 \mu \mathrm{A}$ and $170 \mathrm{~V} / 5.5 \mu \mathrm{A}$, respectively. These results are the best achieved up to date in lead free flexible piezoelectric energy harvesters. The in vivo implementation of the system was carried out into a porcine chest achieving up to $5 \mathrm{~V}$ and $700 \mathrm{nA}$ through the conversion of a continuous heartbeat. The system showed good short-term biocompatibility as demonstrated by cell and histological studies, demonstrating suitable properties for biocompatible and ecofriendly applications [131].

With the intention to produce energy from human limbs motion, a prototype based on a PZT piezoelectric bending structure was designed consisting of a frame containing a channel that guides a free sliding mass and two piezoelectric bimorphs located at the end of the frame. The device with $3.5 \times 2 \times 2 \mathrm{~cm}$ and $60 \mathrm{~g}$, placed on a hand of a person and shaken at $10 \mathrm{~Hz}$ and $10 \mathrm{~cm}$ amplitude movements, produced an output power of $600 \mu \mathrm{W}$. A power output of $47 \mu \mathrm{W}$ was achieved when the harvester was rotated $180^{\circ}$ each second. It was also indicated further system improvements to be made in order to optimize the output performance, such as the selectin of appropriate materials and dimensions to increase the electromechanical coupling factor and the mechanical quality factor, increase 
the resonant frequency sufficiently so that the generated energy can be transferred before the second impact, choose a sliding mass as stiff as possible to avoid storing energy into vibrations instead of the vibrations of the piezoelectric transducer and implement a damping system in the neighborhood of the piezoelectric elements to limit the reliability problems due to high stresses and deflections resulting from high amplitude excitations [113]. On the same line, the inclusion of a unimorph strip of spring steel bonded to a patch of PZT into a shoe sole achieved an output power of $\sim 80 \mathrm{~mW}$. It was estimated that the generation of $10 \mathrm{~mW}$ from the shoe during a useful life of 2 years is equivalent to 150 $\mathrm{cm}^{3}$ of a lithium-thionyl chloride battery [117].

Other ceramic materials, such as $\mathrm{BaTiO}_{3}$ and $\mathrm{ZnO}$, have also been investigated in piezoelectric energy harvesting applications, commonly in the configuration of nanowires (Figure 17) or nanorods [49, 124, 126-129].

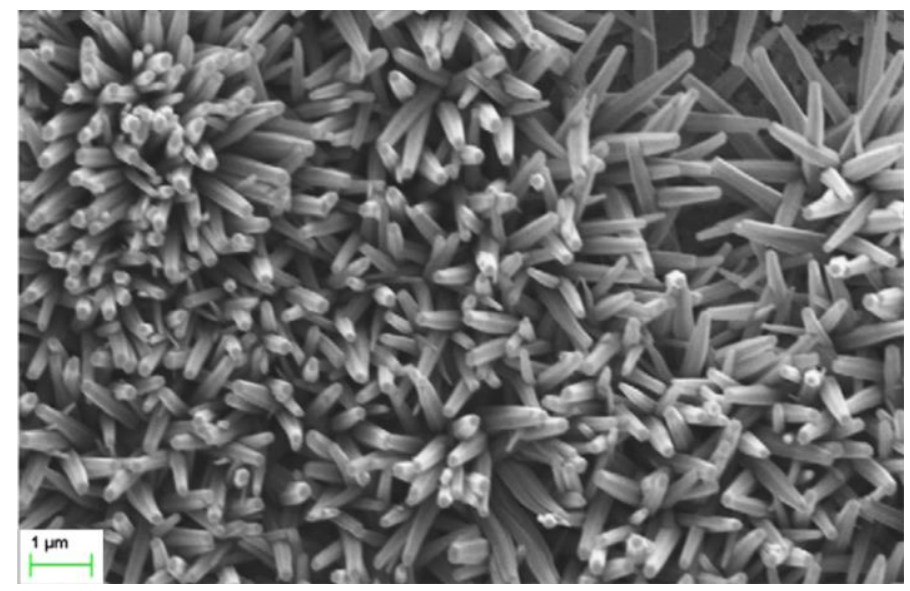

Figure 12: SEM micrograph of $\mathrm{ZnO}$ nanowires [134].

A piezoelectric nanoelectromechanical vibrational energy harvester based on $\mathrm{BaTiO}_{3}$ vertically aligned nanowires arrays $\sim 1 \mu \mathrm{m}$ long, produced by two-step hydrothermal reaction on a fluoride thin oxide glass conductive substrate $(\sim 10 \times \sim 10 \times 2.2 \mathrm{~mm})$, generated an average power of $\sim 125.5 \mathrm{pW}$ at optimal load resistance of $120 \mathrm{M} \Omega$ from $1 \mathrm{~g}$ root mean square acceleration, corresponding to a peak power density of $\sim 6.27 \mu \mathrm{W} / \mathrm{cm}^{3}$ [124]. A similar system, developed by the same procedure, produced ultralong vertically aligned $\mathrm{BaTiO}_{3}$ nanowire arrays with $\sim 40 \mu \mathrm{m}$ in lenght, obtained an output peak power of $\sim 123 \mathrm{pW}$ at a load resistance of $100 \mathrm{M} \Omega$ from an input root mean square acceleration of $0.25 \mathrm{~g}$, resulting in a peak power density of $\sim 192 \mathrm{nW} / \mathrm{cm}^{3}$. The system showed interesting attributes such as compact size, low resonant frequency $(155 \mathrm{~Hz})$ and biocompatibility for biomedical applications working with mechanical vibrations in the low frequency regime [124]. 
The same concept was explored with a $\mathrm{ZnO}$ nanowires blocks integrated into a microenergy harvester with a microelectromechanical proof mass. A single nanowires block had the dimensions of $8 \times 8 \mu \mathrm{m}$ and each nanowire presented the height of $5 \mu \mathrm{m}$ and an average diameter of $150 \mathrm{~nm}$. The system generated a peak power of $70 \mathrm{pW}$ across an optimal load of $7 \mathrm{M} \Omega$ with an acceleration of $1.4 \mathrm{~g}$ and at a frequency of $40 \mathrm{~Hz}$, which corresponds to a $22 \mathrm{~mW} / \mathrm{cm}^{3}$ volume normalized power output [135].

The ceramic $\mathrm{ZnO}$ was also investigated in a nanogenerator based on vertically aligned nanorods, produced by a low temperature hydrothermal method, on poly $(3,4-$ ethylenedioxythiophene) polystyrene sulfonate (PEDOT:PSS) plastic substrate with an area of $3 \times 1 \mathrm{~cm}^{2}$. The system was tested by vibrations/pressure forces and registered an output power of $0.123 \mu \mathrm{W}$ at a frequency of $41 \mathrm{~Hz}$ and a load of $1000 \mathrm{~g}$ [130].

\subsection{Piezoelectric energy harvesting based on single crystals}

Single crystals are the materials in which piezoelectricity was first observed. These materials represent a crystalline counterpart of a piezoelectric ceramic, although single crystals show higher strain constants than ceramics and possesses much lower Young's modulus, which allows lower resonance frequencies with smaller devices. Among the available single crystals, PMN-PT and PZN-PT are the most widely used due to their superior performance $[34,136]$.

The Table 9 summarizes the reports using single crystals in the development of energy harvesting devices.

Table 9: Summary of the single crystals based energy harvesting generators.

\begin{tabular}{|c|c|c|c|}
\hline $\begin{array}{l}\text { Piezoelectric } \\
\text { Material }\end{array}$ & $\begin{array}{c}\text { Output } \\
\text { Performance }\end{array}$ & Characteristics & Ref. \\
\hline \multirow{4}{*}{ PMN-PT } & $5 \mathrm{nW} / \mathrm{g}^{2}$ & \multirow{8}{*}{ Cantilever beam } & [137] \\
\hline & $0.7 \mathrm{~mW}$ & & [138] \\
\hline & $2.16 \mathrm{~mW}$ & & {$[88]$} \\
\hline & $14.7 \mu \mathrm{W}$ & & {$[139]$} \\
\hline \multirow{2}{*}{ PZN-PT } & $1.2 \mathrm{~mW}$ & & [138] \\
\hline & $0.571 \mathrm{~mW}$ & & [96] \\
\hline PIN-PMN-PT & $5.18 \mathrm{~mW}$ & & {$[88]$} \\
\hline AlN & $34.78 \mu \mathrm{W}$ & & {$[140]$} \\
\hline \multirow{5}{*}{ PMN-PT } & $17.8 \mathrm{~V} / 1.74 \mu \mathrm{A}$ & Flexible energy harvester & [141] \\
\hline & $\sim 1.2 \mathrm{~V}$ & Nanobelt generator & [142] \\
\hline & $160 \mathrm{~kJ} / \mathrm{m}^{3}$ & Cyclic piezoelectric harvester & {$[143]$} \\
\hline & $8.2 \mathrm{~V} / 145 \mu \mathrm{A}$ & Thin film to self-power cardiac pacemaker & [144] \\
\hline & $3.7 \mathrm{~mW}$ & Cantilever beam combined with cymbal transducer & [145] \\
\hline $\mathrm{LiNbO}_{3}$ & $16.5 \mathrm{nW}$ & High temperature harvester & [146] \\
\hline PIN-PMN-PT & $6.22 \mathrm{~kJ} / \mathrm{m}^{3}$ & Compressive cyclic stress & [147] \\
\hline
\end{tabular}




\begin{tabular}{|l|c|c|c|}
\hline & $750 \mu \mathrm{J} / \mathrm{cm}^{3}$ & Cyclic stressed harvester & {$[148]$} \\
\hline
\end{tabular}

Among single crystals based energy harvesting devices, the cantilever beams configuration continues to be repeatedly used to produce vibration energy harvester. A cantilever beam with a tip mass was developed with the single crystal PMN-PT with (67:33) composition as piezoelectric material, grown via a vertical gradient freeze method. The system was excited under an acceleration of $1 g$ at a resonance frequency of $50 \mathrm{~Hz}$ and the maximum power obtained was $5 \mathrm{nW} / \mathrm{g}^{2}$ for an optimum resistive load of 2 $\mathrm{M} \Omega$, with a cantilever of approximately $0.300 \mathrm{~cm}^{3}$ and $6.03 \mathrm{~g}$. The system was tested to recharge a CMOS lithium battery with nominal operating voltage of $3.0 \mathrm{~V}$, and at the resonance frequency and optimum resistance the output voltage increased up to a constant value of $2.88 \mathrm{~V}$, which corresponds to approximately $92 \%$ of the full charge of the rechargeable battery [137]. A comparison study of the energy harvesting efficiency between PMN-PT, PZN-PT and the ceramic PZT cantilever beams was performed, and both single crystals based systems showed higher performance than the ones fabricated from PZT. Among single crystals, the PZN-PT harvester showed the highest root mean square power output with $1.2 \mathrm{~mW}\left(\mathrm{R}_{\mathrm{L}}=600 \mathrm{k} \Omega\right)$ whereas the PMN-PT harvester registered $0.7 \mathrm{~mW}\left(\mathrm{R}_{\mathrm{L}}=500 \mathrm{k} \Omega\right)$ at $0.5 \mathrm{~g}$ of acceleration [138].

The concept of MEMS energy harvester has also been explored with a cantilever beam based on PZN-8\% PT single crystals. The micro-generator composed of piezoelectric beams with dimensions of $5 \times 1 \times 0.4 \mathrm{~mm}$, with a piezoelectric layer with thickness of $0.1 \mathrm{~mm}$, registered a power of $0.571 \mathrm{~mW}$ at an external resistance of $215 \mathrm{k} \Omega$ [96].

In an attempt to benefit from the advantages of the cantilever beam and cymbal transducer configurations, a combination of both in one device was reported as an effective way to improve the properties of piezoelectric energy harvesters. The cymbal transducers were produced from a $0.71 \mathrm{PMN}-0.29 \mathrm{PT}$ single crystal ingot grown directly from the melt by a modified Bridgman technique and diced into (001) oriented plates of $25 \times 5 \times 1 \mathrm{~mm}$ and sandwiched between two protruded stainless steel (SUS 304) caps, and the cantilever was made of a beryllium copper (C17200) slice with $35 \times 5 \times 0.5 \mathrm{~mm}$ with a proof mass of $4.2 \mathrm{~g}$. The structures were assembled and clamped by an aluminum alloy frame to form a composite structure similar that shown in Figure 18. The system generated a maximum power of $3.7 \mathrm{~mW}$ under an excitation of $3.2 \mathrm{~g}$ at the resonant frequency of $102 \mathrm{~Hz}$ with a matching load resistance of $251 \mathrm{k} \Omega$. The experimental results showed that the use of 
PMN-PT single crystal material allowed lower the resonance frequency and increased significantly the output power [145].

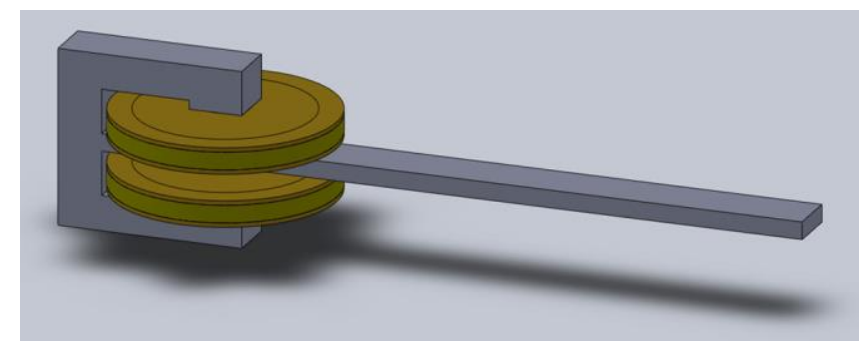

Figure 13: Schematic representation of an assembling of a cantilever beam between two cymbal transducers [109].

An approach in the scope of in vivo energy harvesting reported the production of a harvester based on (001) oriented 0.72PMN-0.28PT thin film onto a flexible substrate of PET $(110 \mu \mathrm{m})$ to achieve a self-powered artificial pacemaker. The maximum output current and voltage was $145 \mathrm{nA}$ and $8.2 \mathrm{~V}$ through periodic mechanical motions bending and unbending, respectively. Moreover, $223 \mu \mathrm{A}$ were obtained by finger tapping, corresponding to the highest output current among flexible piezoelectric base nanogenerators. Real-time functional electrical stimulation was performed to provide the artificial heart beating for a live rat using the flexible PMN-PT energy harvester, and with a rat heart beat rate of 6 beats per second, the cyclically stimulation generated an energy of $2.7 \mu \mathrm{J}$ [144]. Still within the scope of in vivo applications a flexible energy harvester was fabricated based on a thin single-crystalline PMN-PZT film, doped with $0.5 \mathrm{~mol} \%$ of Mn, with $3.5 \times 7 \mathrm{~cm}$ and $20 \mu \mathrm{m}$ of thickness fixed onto a PET substrate with $3.5 \mathrm{~cm} \times$ $7 \mathrm{~cm} \times 75 \mu \mathrm{m}$ coated with an adhesive epoxy. The flexible energy harvester was implanted in a porcine heart and generated an open-circuit voltage of $17.8 \mathrm{~V}$ and a shortcircuit current of $1.74 \mu \mathrm{A}$ from the porcine heartbeats. The biocompatibility of the system was proved by a cell viability test and histological analysis. Finally, the energy obtained from cardiac motions was used to wirelessly turned on and off a light bulb to visually verify the data transmission [141].

Another approaches using less common single crystals can be found in the literature, such as an AlN based cantilever with an output of $34.78 \mu \mathrm{W}$ at a resonant frequency of 572 $\mathrm{Hz}$, an acceleration of $2 g$, an optimal resistance of $495 \mathrm{k} \Omega$ and with a seismic mass of 5.6 $\times 5.6 \mathrm{~mm}$ [140], and a high temperature harvester based on a Y-cut $\mathrm{LiNbO}_{3}(010)$ crystal 
that generated a maximum output power of $16.5 \mathrm{nW}$ across a resistance load of $0.8 \mathrm{M} \Omega$ and at $400{ }^{\circ} \mathrm{C}$ [146].

\subsection{Piezoelectric energy harvesting based on polymers}

Piezoelectric polymers are flexible and deformable materials that allow an easy assembling to curved surfaces and at the same time are resistant to mechanical impacts. In relation to piezoceramics, piezopolymers present significant lower density and higher flexibility, in turn their piezoelectric constants are significant lower. The most common piezoelectric polymer used in energy harvesting applications is the fluoropolymer PVDF and its copolymer P(VDF-TrFE), essentially due its flexibility, adequate mechanical strength, ease of processing and high chemical resistance. Further, PVDF is chemically stable and biocompatible which is an advantage regarding its application in biological systems $[34,82,83]$. The number of studies available in the literature dedicated to PVDF polymers attests its importance for this kind of applications. In the Table 10 are summarized several reports devoted to energy harvesting from polymers.

Table 10: Summary of piezoelectric energy harvesters based on polymers.

\begin{tabular}{|c|c|c|c|}
\hline $\begin{array}{c}\text { Piezoelectric } \\
\text { Material }\end{array}$ & $\begin{array}{c}\text { Output } \\
\text { Performance }\end{array}$ & Characteristics & Ref. \\
\hline \multirow{15}{*}{ PVDF } & $0.35 \mu \mathrm{W}$ & \multirow{6}{*}{ Cantilever beam } & [9] \\
\hline & $\sim 45 \mathrm{~V}$ & & [9] \\
\hline & $3 \mathrm{~V} / 2.5 \mu \mathrm{A}$ & & [149] \\
\hline & $8.1 \mathrm{nW}$ & & [150] \\
\hline & $\sim 600 \mu \mathrm{W}$ & & {$[151]$} \\
\hline & $2.2 \mu \mathrm{W}$ & & {$[152]$} \\
\hline & $\sim 1.3 \mathrm{~V}$ & Human exhaled air harvester & [153] \\
\hline & $200 \mathrm{~mW}$ & Rodway energy harvester & {$[154]$} \\
\hline & $\sim 5.0 \mathrm{~mW} / \mathrm{cm}^{3}$ & \multirow{2}{*}{ Piezoelectric flag } & [155] \\
\hline & $\sim 4.5 \mathrm{~W}$ & & [156] \\
\hline & $2 \mu \mathrm{W}$ & Multilayer harvester & [157] \\
\hline & $20 \mathrm{~mW}$ & Strip for shoes soles & [117] \\
\hline & $200 \mathrm{nW}$ & \multirow{5}{*}{ Nanofiber based generator } & [158] \\
\hline & $\sim 1.3 \mathrm{~V}$ & & [159] \\
\hline & $\sim 0.02 \mu \mathrm{W}$ & & {$[160]$} \\
\hline PLLA & $19.5 \mathrm{nW}$ & & {$[161]$} \\
\hline \multirow{4}{*}{$\mathrm{P}(\mathrm{VDF}-\mathrm{TrFE})$} & $\sim 0.011 \mu \mathrm{W}$ & & [160] \\
\hline & $\sim 0.26 \mu \mathrm{W}$ & Spin coated film generator & [162] \\
\hline & $5.6 \mathrm{~V}$ & Nanowires based generator & [163] \\
\hline & $0.2-1.0 \mu \mathrm{W}$ & Flexible skin-attachable generator & [164] \\
\hline
\end{tabular}

Among the possible configurations to develop energy harvesting systems based on polymers, the cantilever beams stand out similarly to piezoceramics applications. An 
interesting approach has been developed based on a quarter-wavelength straight-tube acoustic resonator $58 \mathrm{~cm}$ long and a cross section of $4 \times 5 \mathrm{~cm}$, with PVDF piezoelectric cantilever beams with $2 \times 1.61 \mathrm{~cm}$ and $0.2 \mathrm{~mm}$ of thickness placed inside (Figure 19a). Each unimorph cantilever was composed of a $30 \mu \mathrm{m}$ thick PVDF film, $127 \mu \mathrm{m}$ thick polyester laminate and an adhesive layer. Through the excitation of the tube, was generated an amplified acoustic resonant standing wave that provokes the vibration of PVDF beams (Figure 19b). At the incident sound pressure level of $110 \mathrm{~dB}$ a voltage of $1.48 \mathrm{~V}$ was obtained which corresponded to $2.2 \mu \mathrm{W}$ and $0.11 \mu \mathrm{W} / \mathrm{cm}^{2}$ [152].

a)

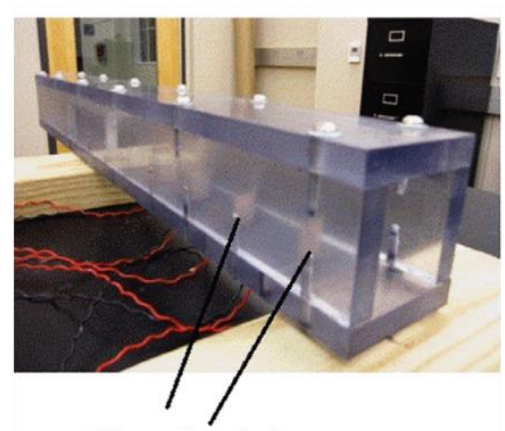

b)

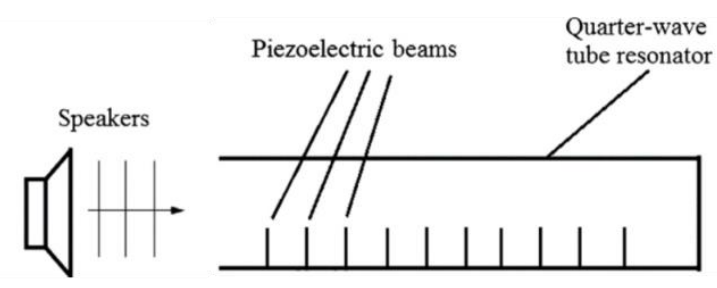

Piezoelectric beams

Figure 19: a) Perspective of the quarter-wavelength tube resonator with the PVDF piezoelectric beam array and a) sketch of the experimental setup [152].

A bi-resonant structure consisting of two cantilever-mass beams $(55 \times 18 \mathrm{~mm})$ of stainless steel with attached PVDF film and a tip mass (Figure 20) has been developed. The system was composed by two cantilevers to achieve two different resonant frequencies, when one mass was oscillating at resonance, the vibration amplitude was large enough to the mass to collide with the other mass and drive the second into a vibration mode. The system was tested with a gap of $0.5 \mathrm{~mm}$ between the top cantilever, with $0.15 \mathrm{~mm}$ of thickness and a tip mass of $1.5 \mathrm{~g}$, and the bottom cantilever with the thickness of $0.2 \mathrm{~mm}$ and a tip mass of $1.2 \mathrm{~g}$ and generated an output power of $0.35 \mu \mathrm{W}$ across an external load resistance of $20 \mathrm{M} \Omega$, at a low operation frequency of $16 \mathrm{~Hz}$ and at $9.81 \mathrm{~m} / \mathrm{s}^{2}$, corresponding to a harvested power density of the dual resonant device of about 1 $\mu \mathrm{W} / \mathrm{cm}^{3}$. The theoretical analysis and experimental results of this study showed that a piezoelectric energy harvester device with a bi-resonant structure could harvest more energy than the sum of the two separated single devices [9].

An innovative approach of a spiral-shaped cantilever beam based on PVDF was developed by micro-fabrication processes. It is stated that this geometry increases the 
effective length of the cantilever which reduces the resonance frequency. The system generated an output power of $8.1 \mathrm{nW}$ at a load resistance of $100 \mathrm{M} \Omega$, corresponding to a power density of $0.81 \mu \mathrm{W} / \mathrm{cm}^{3}$ at the resonant frequency of about $20 \mathrm{~Hz}$ and an acceleration of $0.2 g$ [150].

The harvesting of ambient vibrations, such as blowing wind, have been explored in different approaches, such as a cantilever-pendulums architecture, based on PVDF, harvesting the wind-induced mechanical vibration energy. Different cantilever beams were tested and the best output performance was obtained for a bimorph configuration with the area of $72 \times 16 \mathrm{~mm}$ and the thickness of $0.41 \mathrm{~mm}$, which registered an output power of $\sim 615 \mu \mathrm{W}$ for $8 \mathrm{~m} / \mathrm{s}$ of wind on $5 \mathrm{M} \Omega$ load resistance [151]. Another approach based on the same principle, described a PVDF flag generator $(100 \times 10 \times 0.05 \mathrm{~mm})$ and the maximum output power of the system was dictated by both the optimal resistance and the circuit voltage. It turns out that the optimal resistance was inversely proportional to the inlet velocity, the voltage tending to stabilize around $4 \mathrm{~m} / \mathrm{s}$ and the maximum power increasing persistently reaching values of $\sim 4.5 \mathrm{~W}$ for inlet velocities around $10 \mathrm{~m} / \mathrm{s}$ [156]. In a similar approach, the wind energy harvesting of PVDF piezoelectric flags under controlled wind and ambient conditions was studied. Poled PVDF membranes with thickness of $0.2 \mathrm{~mm}$ and four different aspect ratios were tested in a wind tunnel with cross section of $0.3 \times 0.3 \mathrm{~m}$, capable of producing free-stream velocities between 0 and $10 \mathrm{~m} / \mathrm{s}$, by fixing the trailing edge and leaving the leading edge free to move in a way called inverted flag. A peak power density of $\sim 5.0 \mathrm{~mW} / \mathrm{cm}^{3}$ was generated at the wind velocity of $9 \mathrm{~m} / \mathrm{s}$. The inverted flag configuration presented several advantages such as the self-induced fluttering within a large wind velocity range, the non-dependence of selffluttering on resonance with possibility of tuning adjusting the bending stiffness, and possibility to self-oscillate at a desired wind speed by adjusting its length [155].

In the field of human motion energy harvesting, a multilayer stave based on laminate PVDF, with 2 layers of 8 PVDF sheets of $28 \mu \mathrm{m}$ thickness between a plastic core of 2 $\mathrm{mm}$ thickness, was produced for shoe sole harvesting achieving an output of $20 \mathrm{~mW}$ [117]. An interesting approach in this scope has been developed for the medical equipment spirometer, used to measure the lung capacity of a human being. A piezoelectric film of PVDF with $28 \mu \mathrm{m}$ of thickness was installed into the pipe of the equipment, with $40 \mathrm{~mm}$ of diameter, with the intention to harvest energy from the human exhalation while measurements with the equipment are carried out (Figure 20). Simulation tests using a calibrated syringe of $3 \mathrm{~L}$ were performed and a maximum output 
voltage of $1.1 \mathrm{~V}$ was generated. A pyroelectric effect on the PVDF was reported to increase the voltage from 0.6 to $1.1 \mathrm{~V}$. In real life measurements with participants having different height, weight, age and gender, 3 PVDF films in parallel and series connection were evaluated, and output voltages in the range of 0.25 to $1.3 \mathrm{~V}$ for the parallel configuration was obtained, which was higher than that for the series configuration, moreover the characteristics of the participants was determinant for the output performance. In short, exhaled air piezoelectric energy harvesting was successfully achieved without disturbing the lung parameter measurement with medical spirometer [153].

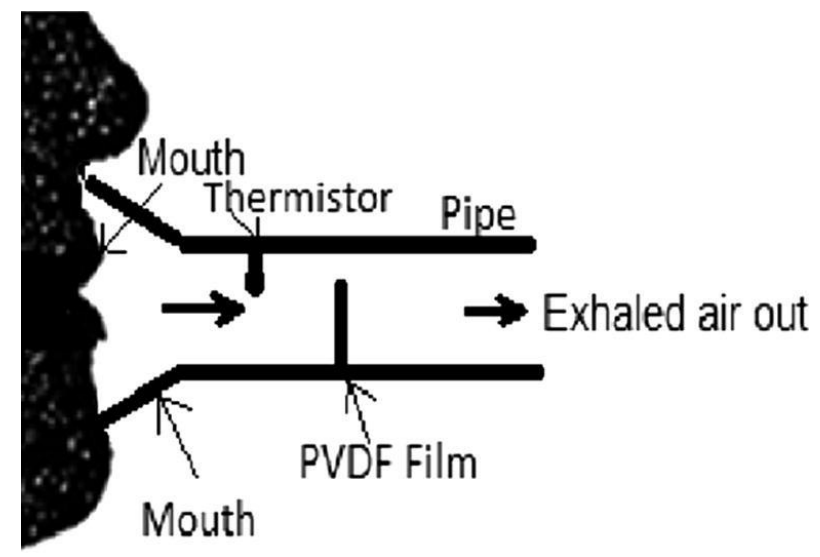

Piece

Figure 14: Schematic representation of the exhalation in the pipe of the spirometer [153].

The PVDF copolymer, $\mathrm{P}(\mathrm{VDF}-\mathrm{TrFE})$, has also been studied and incorporated in different energy harvesting approaches. Similarly to the ceramics, the nanowire configuration was investigated on a flexible nanogenerator based on $\mathrm{P}(\mathrm{VDF}-\mathrm{TrFE})$. The nanowires array was produced by pressing an anodic aluminum oxide nonporous template on a spin coated polymer film with 4-5 $\mu \mathrm{m}$ of thickness. The device was regularly deformed with periodical bending and unbending motions, and for a movement of $10 \mathrm{~mm}$ with an average speed of $20 \mathrm{~mm} / \mathrm{s}$ a positive output voltage of $5.6 \mathrm{~V}$ was registered upon bending states, and a corresponding negative output upon the releasing states. The authors conclude that the output voltages depended on the bending strain rate, which increase with increasing bending speed [163]. The nanofiber configuration was also addressed with a $\mathrm{P}(\mathrm{VDF}-\mathrm{TrFE})$ nanogenerator produced by electrospinning. The nanofibers were electrospuned on interdigitated electrodes plates (Figure 21a) and the energy harvesting performance evaluated by periodic bending tests, with maximum vertical displacements 
about $7 \mathrm{~mm}$ (Figure 22b), generating a maximum power output of $\sim 0.011 \mu \mathrm{W}$ with the load resistance of $1 \mathrm{M} \Omega$ [160].
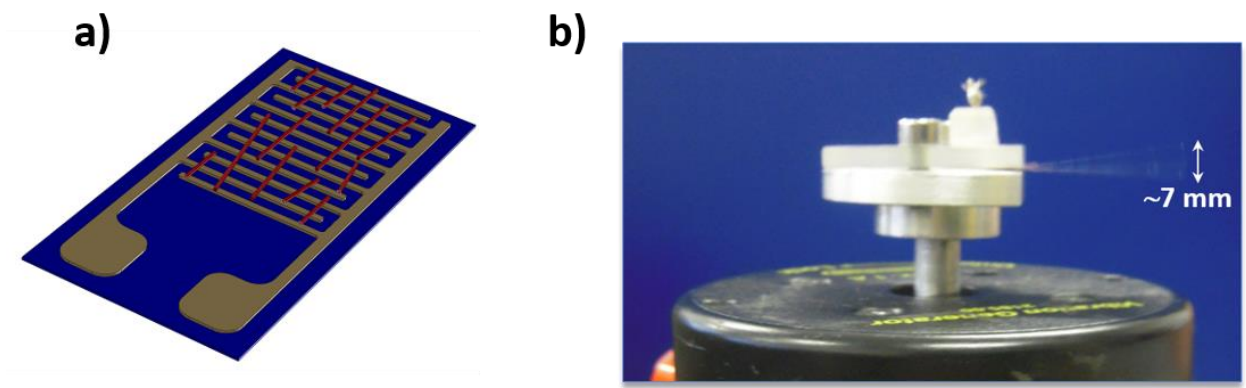

Figure 15: a) Schemamic representation of the interdigitated electrodes plate with electrospun nanofibers and b) electromechanical generator during an energy harvesting bending test [160].

In a different configuration approach, highly ordered concentric circular PVDF nanofibers were produced by electrospinning. The polymer fibers were deposited on a $\mathrm{Cu}$ foil with a diameter of about $4 \mathrm{~cm}$ and glued to a PVC substrate and then encapsulated into PDMS. The system achieved the optimized output power of $200 \mathrm{nW}$ at a resistance of $2 \mathrm{M} \Omega$. According the authors, this concentric configuration was able to harvest mechanical energy regardless the direction of the applied deformation, thereby overcoming the constraint of the aligned fibers of collecting energy in limited direction. Furthermore, the piezoelectric generator was tested for five consecutive days at a constant frequency of $5 \mathrm{~Hz}$ for $10 \mathrm{~min}$ per day, showing negligible variations of the output performance and indicating, therefore, a great stability and robust life time [158]. Electrospinning was used to produce ordered porous piezoelectric nanofibers of poly $\mathrm{L}^{-}$ latic acid) (PLLA) on polyimide substrate, with gold comb electrode sputtered by magnetron sputtering, for strain sensing and human joint motion energy harvesting. The PLLA nanofibers in both pushing and releasing process generated an open circuit voltage of $250 \mathrm{mV}$ and a short-circuit current of $96 \mathrm{pA}$ with pushing displacement of $1 \mathrm{~mm}$. For a strain deformation angle of $28.9^{\circ}$ the system achieved $0.55 \mathrm{~V}$ and $230 \mathrm{nA}$. After longterm continuous operation of 2800 cycles no decline was observed on the output voltage and current thus demonstrating the reliability and durability of the strain sensor. The human joint motion application was also performed to deeper understand the application of the prototype with this purpose, and when the PLLA nanofibers were deformed along a human joint bending, a maximum peak power of $19.5 \mathrm{nW}$ was generated [161].

Also in the scope of human motion energy harvesting, the production of a skin-attachable generator composed by a spin coated $\mathrm{P}(\mathrm{VDF}-\mathrm{TrFE})$ layer on a flexible polyimide support 
with $50 \mu \mathrm{m}$ thicness was reported. The systems is aimed to be attached on a human wrist to harvest energy from the arterial pulsation, and the experiments demonstrated its energy harvesting ability in the range of 0.2 to $1.0 \mu \mathrm{W}$ at a human heart rate range of 50 to 220 beat per minute, from the small skin contact area of $3.71 \mathrm{~cm}^{2}$ where a blood pressure of $40 \mathrm{mmHg}$ was applied [164].

The concept of energy harvesting systems for roadway applications have also been explored for piezoelectric polymers, namely for PVDF. A piezoelectric module with dimensions of $150 \times 150 \times 90 \mathrm{~mm}$ (Figure 22a) was produced with 6 sets of 10 units of energy harvesters parallel connected and fixed in the vertical direction with initial radii of curvature as high as possible, since it was verified by finite element analysis that the vertical deformation was the most effective (Figure 22b). The unit energy harvester consisted of two PVDF films with $110 \mu \mathrm{m}$ of thickness sandwiched into nickel based fabric tapes of $15 \mu \mathrm{m}$ of thickness. The PVDF films were attached to the front and back side of polyimide substrate with $300 \mu \mathrm{m}$ of thickness trough adhesive fabric tapes to complete a bimorph structure (Figure 23c). The module was tested by a continuous load from a tire with axial load applied of $250 \mathrm{kgf}$ per wheel at a speed of $8 \mathrm{~km} / \mathrm{h}$ and generated an instantaneous power output of up to $200 \mathrm{~mW}$ across a load resistance of $40 \mathrm{k} \Omega$, corresponding to a power density of around $8.9 \mathrm{~W} / \mathrm{m}^{2}$. The harvesters exhibited stable performance and durability over repeated number of bending cycles [154].

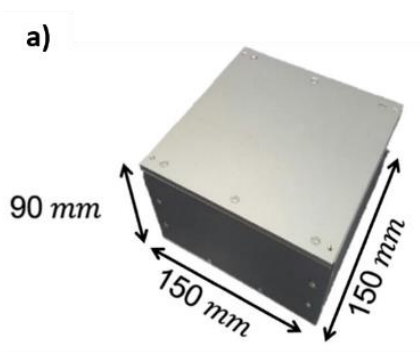

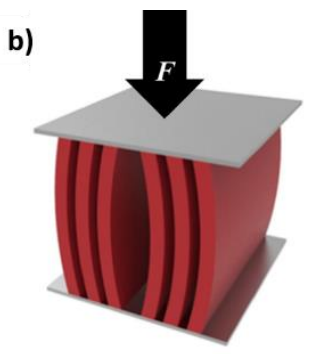

Vertical deformation

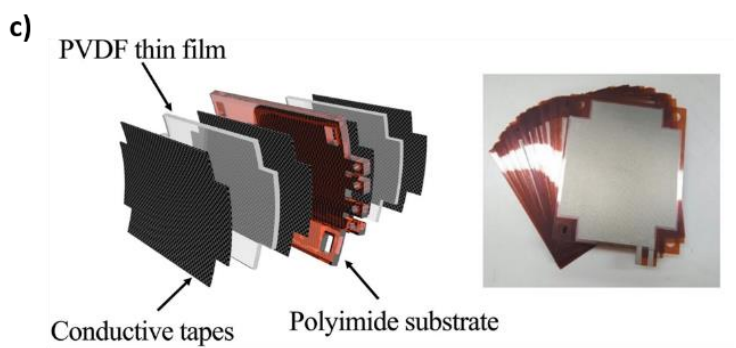

Figure 16: a) Schematic representation of the roadway energy harvester module; b) simple packaging mode of the energy harvester module with the units vertically stacked; c) schematic representation and image of the bimorph energy harvester unit [154].

\subsection{Piezoelectric energy harvesting based on polymer composites}

The use of composites for piezoelectric energy harvesting applications have been studied with the aim to match the best properties of different materials. Typically, most composite materials combine the use of polymers with ceramics to take advantage of the flexibility of the polymers together with the excellent piezoelectric properties of the ceramic 
materials. Table 11 summarizes the energy harvester generators based on composite materials found in recent literature [34].

Table 11: Summary of the composite based energy harvesting generators.

\begin{tabular}{|c|c|c|c|}
\hline Piezoelectric Material & Output Performance & Characteristics & Ref. \\
\hline $\mathrm{ZnO} / \mathrm{PVDF}$ & $\sim 1.0 \mathrm{~V}$ & \multirow{5}{*}{ Nanofiber based generator } & \\
\hline $\mathrm{LiCl} / \mathrm{PVDF}$ & $\sim 1.1 \mathrm{~V}$ & & [159] \\
\hline $\mathrm{BaTiO}_{3} / \mathrm{PDMS}$ & $\sim 60 \mathrm{nW}$ & & [165] \\
\hline $\mathrm{BaTiO}_{3} / \mathrm{P}(\mathrm{VDF}-\mathrm{TrFE})$ & $\sim 0.013 \mu \mathrm{W}$ & & [160] \\
\hline \multirow{2}{*}{$\mathrm{BaTiO}_{3} / \mathrm{PVDF}$} & $0.48 \mathrm{~V}$ & & [166] \\
\hline & $0.072 \mu \mathrm{W}$ & Micro-fabricated power generator & [167] \\
\hline PZT/PET & $185 \mathrm{nW}$ & \multirow{7}{*}{ Spincoated film generator } & \\
\hline $\mathrm{PZT} / \mathrm{RuO}_{2} / \mathrm{PET}$ & $143 \mathrm{nW}$ & & {$[108]$} \\
\hline BiT/PDMS & $0.8 \mathrm{~V}$ & & [169] \\
\hline $\mathrm{BaTiO}_{3} / \mathrm{n}-\mathrm{Gr} / \mathrm{PVDF}$ & $5.8 \mu \mathrm{W}$ & & {$[170]$} \\
\hline $\mathrm{BaTiO}_{3} / \mathrm{P}(\mathrm{VDF}-\mathrm{TrFE})$ & $0.28 \mu \mathrm{W}$ & & [162] \\
\hline \multirow{2}{*}{$\mathrm{BaTiO}_{3} / \mathrm{P}(\mathrm{VDF}-\mathrm{HFP})$} & $0.196 \mu \mathrm{W}$ & & [171] \\
\hline & $\sim 0.48 \mathrm{~W} / \mathrm{cm}^{3}$ & & [172] \\
\hline $\mathrm{ZnO} / \mathrm{PC}$ & $1600 \mathrm{~mW} / \mathrm{m}^{3}$ & \multirow{3}{*}{ Nanowire based generator } & [173] \\
\hline $\mathrm{ZnO/P(VDF-TrFE)}$ & $1.3 \mathrm{~mW} / \mathrm{mm}^{2}$ & & [134] \\
\hline PMN-PT/PDMS & $7.8 \mathrm{~V} / 2.29 \mu \mathrm{A}$ & & [174] \\
\hline PVDF/PDMS & $3.8 \mathrm{~V} / 3.5 \mu \mathrm{A}$ & $\begin{array}{c}\text { Mesoporous nanogenerator for in vivo } \\
\text { biomechanical harvesting }\end{array}$ & [175] \\
\hline PZT/PVDF & $0.35 \mathrm{~V} / \sim 4 \mathrm{nA}$ & Thick film & {$[176]$} \\
\hline PIN-PNM-PT/epoxy 2-2 & $0.28 \mathrm{~mW} / \mathrm{cm}^{3}$ & \multirow{5}{*}{ Flexible film } & [177] \\
\hline $\mathrm{BaTiO}_{3} / \mathrm{PDMS}$ & $7 \mathrm{~mW} / \mathrm{cm}^{2}$ & & [178] \\
\hline $\mathrm{ZnSO}_{3} / \mathrm{PDMS}$ & $20 \mathrm{~V} / 0.6 \mu \mathrm{A}$ & & [179] \\
\hline $\mathrm{Zn}^{2+} / \mathrm{P}(\mathrm{VDF}-\mathrm{HFP})$ & $2.4 \mu \mathrm{W}$ & & [180] \\
\hline $\mathrm{ZnO/PVDF}$ & $\sim 4 \mathrm{~V}($ few nW) & & [181] \\
\hline $\begin{array}{l}\text { PMN-PT/MWCNTs/ } \\
\text { Silicone-rubber }\end{array}$ & $\sim 4 \mathrm{~V} / \sim 500 \mathrm{nA}$ & Highly elastic generator & [182] \\
\hline PMN-PT/Epoxy resin & $7.182 \mu \mathrm{W}$ & \multirow{3}{*}{ Cantilever beam } & [183] \\
\hline PZT-5A/PET & $40 \mu \mathrm{W}$ & & [184] \\
\hline $\begin{array}{l}\mathrm{BaTiO}_{3} / \text { Carbon fiber/ } \\
\text { PDMS }\end{array}$ & $23.5 \mathrm{mV} / \mathrm{g}$ & & [185] \\
\hline $\mathrm{BaTiO}_{3} / \mathrm{P}(\mathrm{VDF}-\mathrm{TrFE})$ & $12.7 \mu \mathrm{W} / \mathrm{cm}^{2}$ & Imprinted micropillars nanogenerator & [186] \\
\hline $\mathrm{BaTiO}_{3} / \mathrm{PDMS}$ & $\sim 6 \mathrm{~V} / \sim 300 \mathrm{nA}$ & Biotemplated flexible nanogenerator & [187] \\
\hline $\mathrm{BaTiO}_{3} / \mathrm{Ca}-\mathrm{alg} / \mathrm{PDMS}$ & $0.4 \mathrm{nW}$ & Self-powered wearable flexion sensor & {$[141]$} \\
\hline $\mathrm{FAPbBr}_{3} / \mathrm{PVDF}$ & $27.4 \mu \mathrm{W} / \mathrm{cm}^{2}$ & Flexible nanogenerator & [188] \\
\hline
\end{tabular}

Energy harvesting systems based on composite materials can be found in literature produced in several configurations, nevertheless it was verified an increasing trend towards systems based on fibers. Thereby, a flexible nanogenerator based on nanofibers of $\mathrm{BaTiO}_{3}$ with diameters from $\sim 100$ to $200 \mathrm{~nm}$ (Figure 23a and b), produced by electrospinning technique combined with a sol-gel process, were deposited on the top of a flexible substrate with interdigital electrodes and covered with PDMS polymer matrix. The system registered an output peak power of $\sim 60 \mathrm{nW}$ under periodic bending excitation 
of $\sim 45 \mathrm{~Hz}$ across a load resistance of $1 \mathrm{M} \Omega$ for a fiber area of $15 \times 8 \mathrm{~mm}^{2}$, and an average power of $6 \mathrm{nW}$ during the measurement period, which according to the authors are relevant and comparable values among lead-free nanogenerators. The small size of the device, the good reliability, high flexibility and sensitivity to external movement al low frequency represent advantages of the system for a broad range of applications [165].
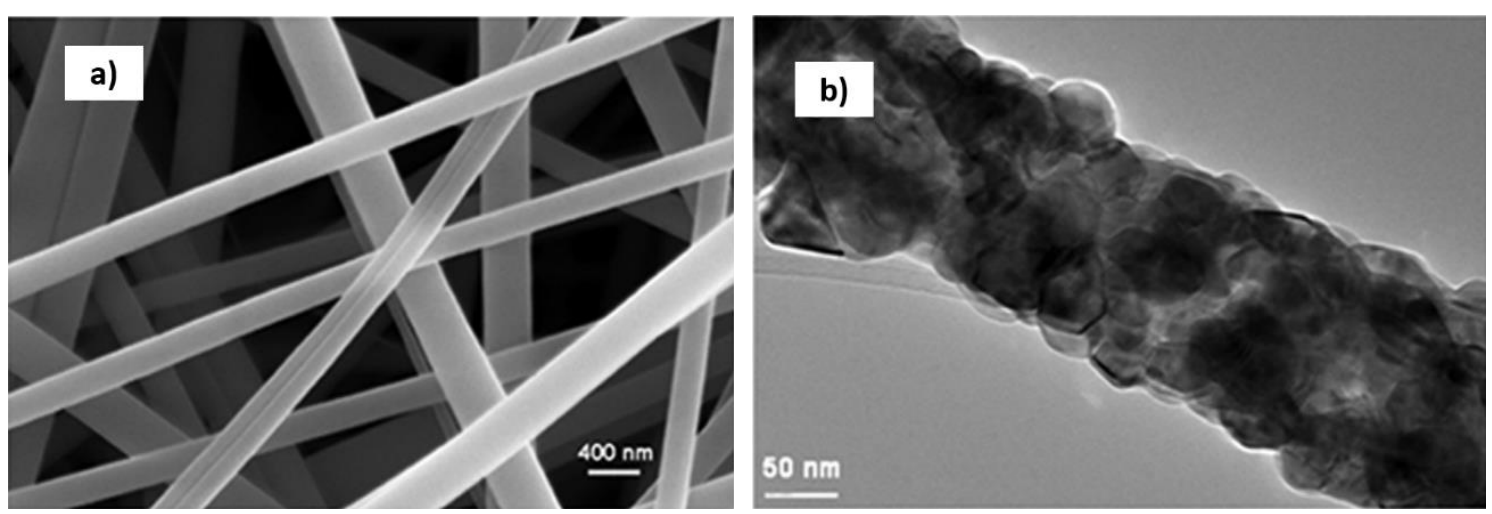

Figure 17: a) SEM and b) TEM images of a $\mathrm{BaTiO}_{3}$ nanofiber [165].

Another electrospun nanofiber generator based on $\mathrm{BaTiO}_{3} / \mathrm{P}(\mathrm{VDF}-\mathrm{TrFE})$ composite reported $\sim 0.013 \mu \mathrm{W}$ of output power under periodic bending tests with maximum vertical displacements of about $7 \mathrm{~mm}$ and a load resistance of $1 \mathrm{M} \Omega$. In this study different $\mathrm{BaTiO}_{3}$ grain sizes were tested $(10,100$ and $500 \mathrm{~nm})$ and it was verified that the composites with the lowest diameter ceramic nanoparticles presented the best performance [160]. A generator composed by highly-aligned electrospun PVDF nanofibers filled with $\mathrm{BaTiO}_{3}$ nanoparticles and with an individual diameter of $\sim 200 \mathrm{~nm}$ (Figure 24) recorded an output voltage of $0.48 \mathrm{~V}$ during the periodic deflection tests at a rate of $0.007 \mathrm{~Hz}$. To perform the tests, ensuring an uniform stress upon deflection, the nanofibers were encased into a PDMS matrix and deflected $6 \mathrm{~mm}$ [166]. 


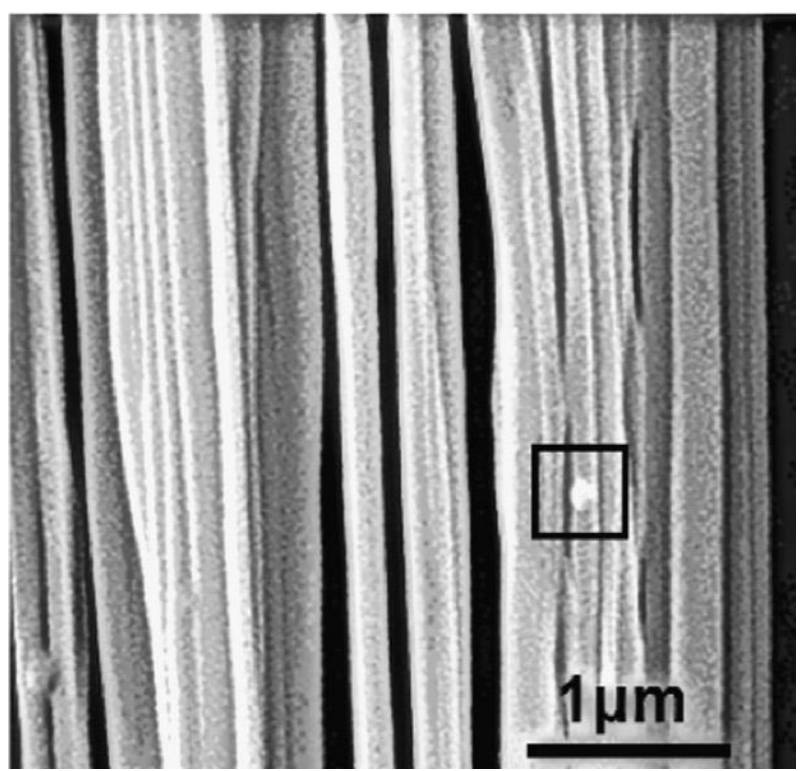

Figure 18: SEM image of highly-aligned nanofibers of $\mathrm{BaTiO}_{3} / \mathrm{PVDF}$ composite with a highlight confirming the presence of $\mathrm{BaTiO}_{3}$ on the surface of the nanofibers [166].

The functionalization of woven fabrics with active coatings have been addressed through the production of multifunctional composites based on carbon fibers covered with $\mathrm{BaTiO}_{3}$ and infiltrated with a PDMS polymer matrix. The woven carbon fibers were coated via two step hydrothermal reaction, the first for the synthesis of $\mathrm{TiO}_{2}$ and the second for the conversion of $\mathrm{TiO}_{2}$ into $\mathrm{BaTiO}_{3}$. The samples were diced to produce cantilever beams and electromechanically tested on a magnet shaker with a tip mass of $1 \mathrm{~g}$. An output of $23.5 \mathrm{mV} / \mathrm{g}$ at the resonant frequency of $96 \mathrm{~Hz}$ was achieved. These materials pave the way to the production of structural woven carbon fiber fabrics with energy harvesting and passive sensing capabilities [185].

The spincoating technique was also used to produced energy harvesting generators based on $\mathrm{BaTiO}_{3} / \mathrm{P}(\mathrm{VDF}-\mathrm{TrFE})$ composites. Piezoelectric thin films with approximately $12 \mu \mathrm{m}$ were spincoated at $500 \mathrm{rpm}$ speed and an acceleration of $750 \mathrm{rpm} / \mathrm{s}^{2}$ during $30 \mathrm{~s}$. Periodic bending tests were performed to test the samples in the range of $1 \mathrm{~Hz}$ to $1 \mathrm{kHz}$ and it was found that the system achieved the maximum output power of $\sim 0.28 \mu \mathrm{W}$ with a load resistance of $1 \mathrm{M} \Omega$ at $1 \mathrm{kHz}$. Similarly to the previous work, different nanoparticle sizes were tested and the best results were found in the lowest particles $(10 \mathrm{~nm})$ [162]. A trilayer nanogenerator with $\sim 60 \mathrm{~nm}$ based on stacked $\mathrm{BaTiO}_{3} / \mathrm{PVDF}$ spincoated layers (at 500 and $1500 \mathrm{rpm}$ ), on each side of a n-graphene layer, achieved a maximum power output of $5.8 \mu \mathrm{W}$ at a load resistance of $1 \mathrm{M} \Omega$. The device presented good stability after 1000 cycles. According to the authors the graphene layer enhance the energy harvesting 
performance of the generator by aligning the dipoles and also increasing the Young's modulus of the piezoelectric film [170].

The ceramic PZT is also reported in composites harvesters, such as the cantilever based on the PZT-5A/PET composite. This system was formed by firmly clamping a piezoelectric bimorph based on PZT-5A to a polymer beam of PET with a proof mass of $0.72 \mathrm{~g}$ (Figure 25a). This original cantilever configuration resulted from the simulation of the voltage distribution in a simple cantilever under a concentric force at the free end (Figure 26b), which showed that the piezoelectric layer towards the free end of the cantilever does not contribute to the energy generation due to negligible strain, thus the piezoelectric bimorph was about $60 \%$ of the total cantilever length. A conventional cantilever system, with the same piezoelectric material, was tested to compare the results. This innovative approach generated the output power of $\sim 40 \mu \mathrm{W}$ at $36 \mathrm{~Hz}$, whereas the conventional cantilever registered $35 \mu \mathrm{W}$ at $125 \mathrm{~Hz}$, both tested at an acceleration of 0.2 $g$ and across a load resistance of $1 \mathrm{M} \Omega$. Thus, this new design favors the decrease of the resonance frequency and increases the output power [184].
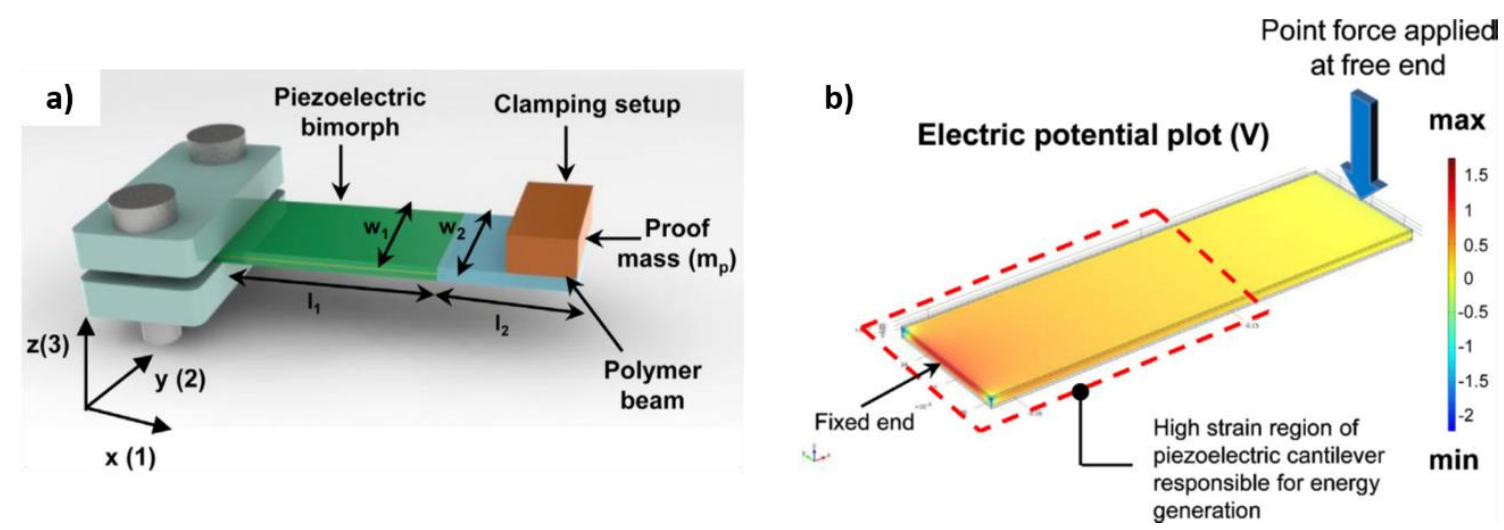

Figure 19: a) Design of the cantilever beam with a polymer spring attached to piezoelectric bimorph; b) Simulation results depicting the voltage generated in the high strain region responsible for energy generation [184].

Composites incorporating single crystals have also been reported, such as the piezoelectric MEMS cantilever harvester based on PMN-PT/epoxy resin composite with interdigitated electrodes, developed by micro-fabrication processes. The harvesters generated the maximum power of $7.182 \mu \mathrm{W}$ at the resonance frequency of $406.0 \mathrm{~Hz}$ and vibration level of $1.5 \mathrm{~g}$, corresponding to a power density of $17181.8 \mu \mathrm{W} / \mathrm{cm}^{3}$ [183]. The single crystal PMN-PT was also prepared in the configuration of nanowires, synthesized by hydrothermal process and mixed with PDMS, to produce a composite nanogenerator 
made of PMN-PT/PDMS with an area of $1 \times 0.5 \mathrm{~cm}^{2}$ and $150 \mu \mathrm{m}$ of thickness. The system was tested by tapping the device vertically using a plastic handle and provided an output voltage of $7.8 \mathrm{~V}$ and a current of $2.29 \mu \mathrm{A}$, achieving a current density of $4.58 \mu \mathrm{A} / \mathrm{cm}^{2}$. The device showed a linear relationship between the output voltage and strain with high sensitivity [174]. Nanowires of ceramic $\mathrm{ZnO}$ with high aspect ratio ( $250 \mathrm{~nm}$ diameter and $\sim 12 \mu \mathrm{m}$ lenght), processed via template-assisted hydrothermal synthesis method, were grown within nanoporous PC templates to produce a piezoelectric nanogenerator with an area of $3 \mathrm{~cm}^{2}$ and a thickness of $\sim 12 \mu \mathrm{m}$, which generated a peak power density of $\sim 1600 \mathrm{~mW} / \mathrm{m}^{3}$ at a load resistance of $\sim 1 \mathrm{M} \Omega$. The system showed an excellent fatigue performance with relative immunity to detrimental environmental factors and mechanical failure since the nanowires remained protected inside the polymer matrix [173].

An innovative approach presented a tri-layer flexible handwriting driven nanogenerator consisting of a $\mathrm{ZnO} / \mathrm{P}(\mathrm{VDF}-\mathrm{TrFE})$ composite ink sandwiched between two foils of $\mathrm{ZnO}$ nanowires chemically grown onto soft porous paper substrates. The system achieved an open circuit voltage of $4.8 \mathrm{~V}$ and a current of $14.4 \mathrm{~mA}$, corresponding to the maximum power density of $1.3 \mathrm{~mW} / \mathrm{mm}^{2}$ connected to a load resistance of $100 \Omega$, for a fast handwriting mode of 200-240 letters/min. These results were stable and reproducible, representing a scalable approach to develop new applications [134]. Another interesting approach based on $\mathrm{BaTiO}_{3} / \mathrm{P}(\mathrm{VDF}-\mathrm{TrFE})$ nanocomposite micropillars explored the fabrication of nanogenerators trough imprinting technique. The ceramic particles were mixed with the copolymer and the obtained solution spin coated on a flexible bottom electrode, then a PDMS mold was hot pressed against the nanocomposite thin film above its melting temperature resulting in the formation of a micropillar array inside. The micropillars were then spin coated with a new PDMS matrix to insulate the system with an effective area of $1 \times 1 \mathrm{~cm}^{2}$. To test the nanogenerators, the device was repeatedly compressed by a vibration exciter with a maximum force of $50 \mathrm{~N}$ at $1 \mathrm{~Hz}$. The maximum output achieved was $13.2 \mathrm{~V}$ and $0.33 \mu \mathrm{A}$ across a load resistance of $3.9 \mathrm{M} \Omega$, corresponding to a power density of $12.7 \mu \mathrm{W} / \mathrm{cm}^{2}$ [186].

Other less common composites such as the one based on PVDF with formamidinium lead halide perovskite $\left(\mathrm{FAPbBr}_{3}\right)$ nanoparticles can be found in literature with significant results. A $\mathrm{FAPbBr}_{3} / \mathrm{PVDF}$ based composite film with $1.2 \times 1.4 \mathrm{~cm}^{2}$ was integrated between PET films with 25 and $125 \mu$ m of thickness, with $\mathrm{Cr}(10 \mathrm{~nm})$ and $\mathrm{Au}(90 \mathrm{~nm})$ thin layers deposited as electrodes. The system was tested with a seismic shaker on mechanical bending measurements and an output voltage of $30 \mathrm{~V}$ and a current density 
of $6.2 \mu \mathrm{A} / \mathrm{cm}^{2}$ under a vertical stress of about $0.5 \mathrm{MPa}$ at $5 \mathrm{~Hz}$ were achieved. The power density increased linearly from 0.87 to $27.4 \mu \mathrm{W} / \mathrm{cm}^{2}$ with increasing load impedance from 0.87 to $500 \mathrm{k} \Omega[188]$.

The in vivo energy harvesting concept has been also explored for composites based generators, in the case a sponge-like mesoporous PVDF film of $1 \times 2 \mathrm{~cm}^{2}$ area and 100 $\mathrm{nm}$ of thickness encapsulated within two distinct PDMS layers of $100 \mu \mathrm{m}$ and $1 \mathrm{~mm}$ of thickness, with interdigitated surface gold electrodes. The system was previously tested under periodical deflecting at $20 \mathrm{~Hz}$ and a force of $6 \mathrm{~N}$ reaching $3.8 \mathrm{~V}$ and $3.5 \mu \mathrm{A}$. Then, the in vivo biomechanical energy harvesting tests were conducted in the quadriceps femoris muscle surface of living rats (Figure 27a): firstly, the animals were under anesthesia and its leg manually deflected, the average output of the harvester was $0.26 \mathrm{~V}$ and $0.17 \mu \mathrm{A}$; afterward, the device was left within the rats during 5 days and the output measured every day having been recorded voltage peaks between 0.21 and $0.26 \mathrm{~V}$ (Figure 26b), which showed the device could operate normally without decay. The biocompatibility of the composite was assessed and no toxicity was found [175].

a)

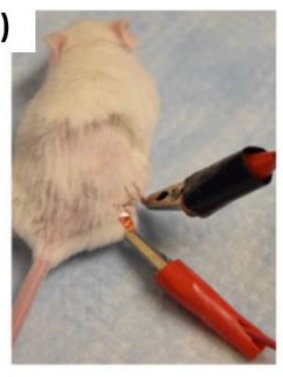

b)

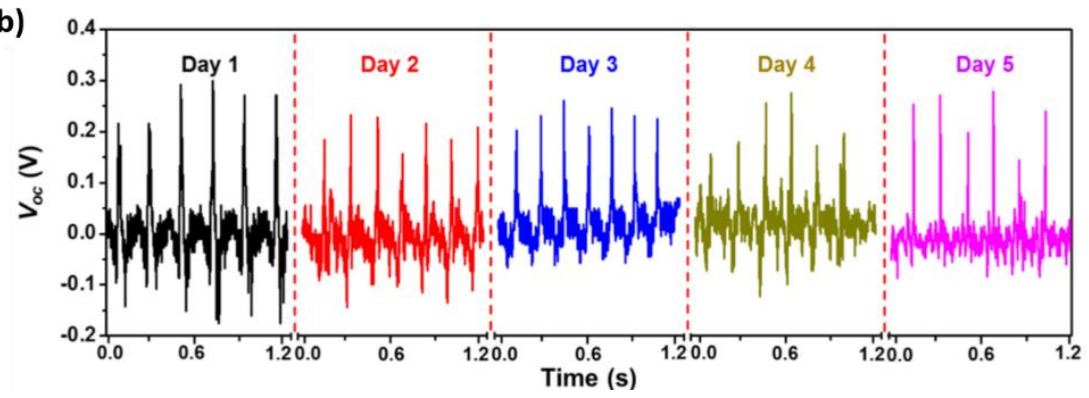

Figure 20: a) Photograph of the connection setup during the in vivo measurements; b) voltage output of the composite during 5 days while the rats live under normal conditions [175].

A self-powered wearable flexion sensor based on spherical composite beads of $\mathrm{BaTiO}_{3}$ and calcium alginate polymer (Ca-alg), prepared by ionotropic gelation method, incorporated into a PDMS matrix was tested for decoding the arm and finger flexion/extension periodic movements, without external battery and sensory circuit (Figure 27). The system, with an area of $7.5 \times 1.5 \mathrm{~cm}^{2}$ and $\sim 20 \mu \mathrm{m}$ of thickness, generated output peak powers from $1.23 \mathrm{pW}$ to $0.4 \mathrm{nW}$ for flexion/extension of the right arm fingers [141]. 
a)

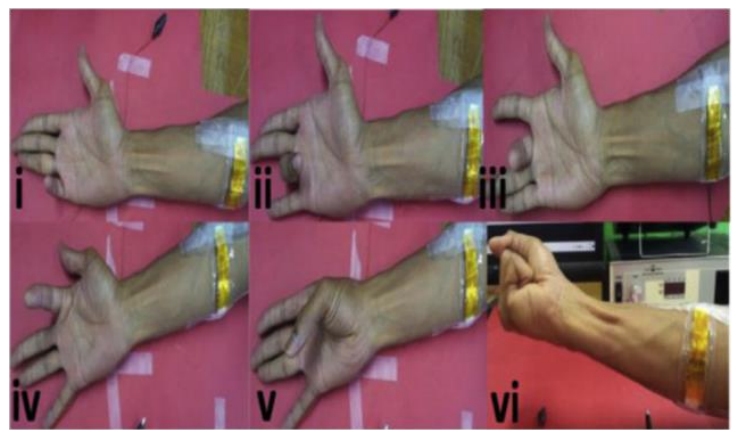

b)

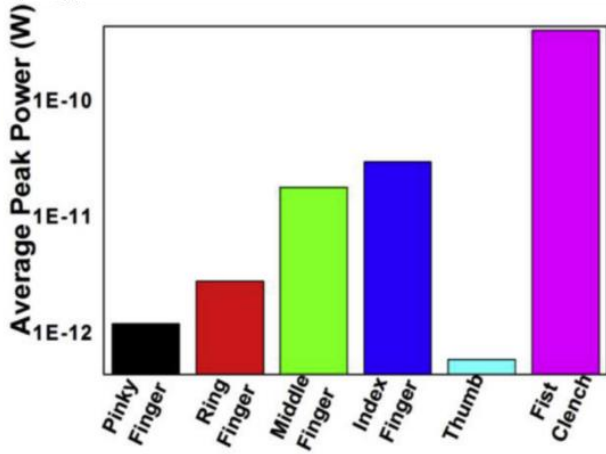

Figure 21: a) Photographs of the fingers and clenched fist movements; b) average peak powers of the flexible sensor during the fingers and clenched fist movements [141].

An innovative approach proposed a flexible nanogenerator based on $\mathrm{BaTiO}_{3} / \mathrm{PDMS}$ synthesized on a M13 virus template. It was found that the barium and titanium precursor ions were strongly associated with the $\mathrm{E} 3$ peptide via metal ion and the virus-metal ionic complexes were used to synthesize virus-templated $\mathrm{BaTiO}_{3}$ nanostructures. After, the virus-templated $\mathrm{BaTiO}_{3}$ crystals were mixed into PDMS and spin-casted onto PDMS/ITO $(100 \mathrm{~nm}) /$ PET $(175 \mu \mathrm{m})$ flexible substrate at $1500 \mathrm{rpm}$. The device with an effective area of $2.5 \times 2.5 \mathrm{~cm}^{2}$ was tested by periodical bending/releasing motion at $0.3 \mathrm{~Hz}$, leading to an output voltage of $\sim 6 \mathrm{~V}$ and a current $\sim 300 \mathrm{nA}$ [187].

\section{Future directions}

Piezoelectric energy harvesting is a concept of high interest and wide application potential in the near future for a broad range of low power electronics devices. Further developments and optimizations are expected in piezoelectric materials for powering sensors in remote and hard-to-reach locations operating in a discontinuously mode, for self-powered sensors operating in body thus avoiding difficult maintenance for patients, for wearable sensors for monitoring vital functions in several activities and for powering portable gadgets replacing a hundred number of accessories that make it difficult to use day to day, among others. On the other hand, the research in piezoelectric materials are in continuous progress to improve their intrinsic properties such as electromechanical coupling conversion efficiency, temperature and chemical stability. Finally, in some cases it is also needed the development of more efficient production and system integration technologies in order to carry out a suitable transition from prototypes to products. 


\section{Conclusions}

After a short introduction to the main concepts related to piezoelectric energy harvesting, this chapter provided an overview of the main piezoelectric materials with potential applicability for electromechanical energy harvesting. The different classes of materials have been discussed, including ceramics, single crystals, polymers and polymer composites. The main properties and limitations of the different material classes have been presented together with the main relevant characteristics for piezoelectric applications. Finally, the main energy harvesting results have been summarized for the different classes of materials showing that piezoelectric energy harvesting devices hold great potential for collecting useful energy from wasted sources, such as mechanical vibrations, that can be used to power micro devices. Strong research efforts are being devoted in order to improve materials response and processing technologies, allowing improved performance and integration, which will open important application areas for these piezoelectric energy harvesting devices in the fields of low power portable electronics and wearable sensors, self-powered sensors and sensors for remote or difficult to reach locations. 


\section{Acknowledgements}

The authors thank Foundation for Science and Technology (FCT) in the framework of the Strategic Funding UID/FIS/04650/2013 (POCI-01-0145-FEDER-006941) funded by national funds through Fundação para a Ciência e a Tecnologia (FCT) and by FEDER funds through COMPETE2020 - Programa Operacional Competitividade e Internacionalização (POCI). PC thanks the FCT gor the SFRH/BPD/110914/2015 grant. JNP wish to thank the financial support of the project Centro-01-0145-FEDER-000017 EMaDeS - Energy, Materials and Sustainable Development, co-financed by the Portugal 2020 Program (PT 2020), within the Regional Operational Program of the Center (CENTRO 2020) and the European Union through the European Regional Development Fund (ERDF). The authors acknowledge funding by the Spanish Ministry of Economy and Competitiveness (MINECO) through the project MAT2016-76039-C4-3-R (AEI/FEDER, UE) and from the Basque Government Industry Department under the ELKARTEK program. 


\section{References}

1. Selvan, K.V. and M.S.M. Ali, Micro-scale energy harvesting devices: review of methodological performances in the last decade. Renewable \& Sustainable Energy Reviews, 2016. 54: p. 1035-1047.

2. Elvin, N. and A. Erturk, Advances in Energy Harvesting Methods. 2013: Springer New York.

3. $\quad$ Kang, M.-G., et al., Recent progress on PZT based piezoelectric energy harvesting technologies. Actuators, 2016. 5(1): p. 5.

4. Koh, S.J.A., et al., Dielectric elastomer generators: how much energy can be converted? IEEE/ASME Transactions on Mechatronics, 2011. 16(1): p. 33-41.

5. Beeby, S. and N. White, Energy Harvesting for Autonomous Systems. 2010: Artech House.

6. Thomas, J.P., M.A. Qidwai, and J.C. Kellogg, Energy scavenging for smallscale unmanned systems. Journal of Power Sources, 2006. 159(2): p. 1494-1509.

7. Cha, Y.S., et al., Energy harvesting from a piezoelectric biomimetic fish tail. Renewable Energy, 2016. 86: p. 449-458.

8. Lu, Q.Q., et al., Dynamic responses of SMA-epoxy composites and application for piezoelectric energy harvesting. Composite Structures, 2016. 153: p. 843850.

9. $\quad$ Li, S.S., et al., Bi-resonant structure with piezoelectric PVDF films for energy harvesting from random vibration sources at low frequency. Sensors and Actuators a-Physical, 2016. 247: p. 547-554.

10. Harb, A., Energy harvesting: sate-of-the-art. Renewable Energy, 2011. 36(10): p. 2641-2654.

11. Siddique, A.M., S. Mahmud, and B. Van Heyst, A comprehensive review on vibration based micro power generators using electromagnetic and piezoelectric transducer mechanisms. Energy Conversion and Management, 2015. 106: p. 728-747.

12. Vatansever, D., E. Siores, and T. Shah, Alternative Resources for Renewable Energy: Piezoelectric and Photovoltaic Smart Structures, in Global Warming Impacts and Future Perspective, B.R. Singh, Editor. 2012, InTech: Rijeka. p. Ch. 10.

13. Proto, A., et al., Nanogenerators for human body energy harvesting. Trends in Biotechnology, 2017. 35(7): p. 610-624.

14. Jiménez, R. and B. Jiménez, Pyroelectricity in Polycrystalline Ferroelectrics, in Multifunctional Polycrystalline Ferroelectric Materials: Processing and Properties. 2011, Springer Netherlands: Dordrecht. p. 573-616.

15. Zhang, S.J. and F. Li, High performance ferroelectric relaxor-PbTiO3 single crystals: Status and perspective. Journal of Applied Physics, 2012. 111(3): p. 031301.

16. Galassi, C., Advances in Processing of Bulk Ferroelectric Materials, in Multifunctional Polycrystalline Ferroelectric Materials: Processing and Properties. 2011, Springer Netherlands: Dordrecht. p. 1-37.

17. Xu, Y., Ferroelectric Materials and Their Applications. 2013: Elsevier Science.

18. Dineva, P., et al., Piezoelectric Materials, in Dynamic Fracture of Piezoelectric Materials: Solution of Time-Harmonic Problems via BIEM. 2014, Springer International Publishing: Cham. p. 7-32.

19. Erturk, A. and D.J. Inman, Introduction to Piezoelectric Energy Harvesting, in Piezoelectric Energy Harvesting. 2011, John Wiley \& Sons, Ltd. p. 1-18. 
20. Platt, S.R., S. Farritor, and H. Haider, On low-frequency electric power generation with PZT ceramics. Ieee-Asme Transactions on Mechatronics, 2005. 10(2): p. 240-252.

21. Guo, L. and Q. Lu, Potentials of piezoelectric and thermoelectric technologies for harvesting energy from pavements. Renewable and Sustainable Energy Reviews, 2017. 72: p. 761-773.

22. Sodano, H.A., D.J. Inman, and G. Park, A review of power harvesting from vibration using piezoelectric materials. The Shock and Vibration Digest, 2004. 36(3): p. 197-205.

23. Khaled, S.R., D. Sameoto, and S. Evoy, A review of piezoelectric polymers as functional materials for electromechanical transducers. Smart Materials and Structures, 2014. 23(3): p. 033001.

24. Wei, C. and X. Jing, A comprehensive review on vibration energy harvesting: modelling and realization. Renewable and Sustainable Energy Reviews, 2017. 74: p. 1-18.

25. Cheng, L.-Q. and J.-F. Li, A review on one dimensional perovskite nanocrystals for piezoelectric applications. Journal of Materiomics, 2016. 2(1): p. 25-36.

26. Nakamura, K., Ultrasonic Transducers: Materials and Design for Sensors, Actuators and Medical Applications. 2012: Woodhead Publishing.

27. Zhou, Q., et al., Piezoelectric single crystals for ultrasonic transducers in biomedical applications. Prog Mater Sci, 2014. 66: p. 87-111.

28. Gupta, S.M., C.S. Harendranath, and A.R. Kulkarni, Synthesis and characterisation of lead magnesium niobate having exceptionally high dielectric constant. Ceramics International, 1997. 23(3): p. 191-196.

29. Kong, L.B., et al., Lead zinc niobate (PZN)-barium titanate (BT) ceramics from mechanochemically synthesized powders. Materials Research Bulletin, 2002. 37(15): p. 2491-2498.

30. Osamu, F., et al., Dielectric properties of modified lead zinc niobate ceramic. Japanese Journal of Applied Physics, 1985. 24(S3): p. 96.

31. Ye, Z.G., Handbook of Advanced Dielectric, Piezoelectric and Ferroelectric Materials: Synthesis, Properties and Applications. 2008: Elsevier Science.

32. Philco, C., D. Technical Publications, and J.P. Buchanan, Handbook of piezoelectric crystals for radio equipment designers. 1957, Wright-Patterson Air Force Base, Ohio: Wright Air Development Center, Air Research and Development Command, U.S. Air Force.

33. Whatmore, R.W., Pyroelectric devices and materials. Reports on Progress in Physics, 1986. 49(12): p. 1335-1386.

34. Li, H.D., C. Tian, and Z.D. Deng, Energy harvesting from low frequency applications using piezoelectric materials. Applied Physics Reviews, 2014. 1(4): p. 041301.

35. Jiang, X.N., J. Kim, and K. Kim, Relaxor-PT single crystal piezoelectric sensors. Crystals, 2014. 4(3): p. 351-376.

36. Aluminum nitride (AlN) piezoelectric constants, electromechanical coupling factor, in Group IV Elements, IV-IV and III-V Compounds. Part a - Lattice Properties, O. Madelung, U. Rössler, and M. Schulz, Editors. 2001, Springer Berlin Heidelberg: Berlin, Heidelberg. p. 1-4.

37. Harada, Y. and Y. Ikuhara, Handbook of Advanced Ceramics: Chapter 1.1.1. The Latest Analytical Electron Microscope and its Application to Ceramics. 2013: Elsevier Science. 
38. Farrell, R., et al., High temperature annealing studies on the piezoelectric properties of thin aluminum nitride films. MRS Proceedings, 2011. 1052.

39. Yue, W. and J. Yi-jian, Crystal orientation dependence of piezoelectric properties in $\mathrm{LiNbO}_{3}$ and $\mathrm{LiTaO}_{3}$. Optical Materials, 2003. 23(1-2): p. 403-408.

40. Tomoaki, Y., I. Hiroshi, and N. Nobukazu, Piezoelectric and elastic properties of $\mathrm{LiTaO}_{3}$ : temperature characteristics. Japanese Journal of Applied Physics, 1969. 8(9): p. 1127.

41. Chaudhari, V.A. and G.K. Bichile, Synthesis, structural, and electrical properties of pure $\mathrm{PbTiO}_{3}$ ferroelectric ceramics. Smart Materials Research, 2013. 2013: p. 9.

42. Shrout, T.R. and S.J. Zhang, Lead-free piezoelectric ceramics: Alternatives for PZT? Journal of Electroceramics, 2007. 19(1): p. 113-126.

43. Buchanan, R.C., Ceramic Materials for Electronics, Third Edition. 2004: Taylor \& Francis.

44. Chaisan, W., et al., Dielectric and ferroelectric properties of lead zirconate titanate-barium titanate ceramics prepared by a modified mixed-oxide method. Materials Chemistry and Physics, 2007. 104(1): p. 113-118.

45. Okayasu, M. and K. Bamba, Domain switching characteristics of lead zirconate titanate piezoelectric ceramics on a nanoscopic scale. Journal of the European Ceramic Society, 2017. 37(1): p. 145-159.

46. Piezoelectric Materials, in Piezoelectric Materials and Devices. 2012, CRC Press. p. 39-56.

47. Takenaka, T., Chapter 6.2 - Lead-Free Piezoelectric Ceramics A2 - Somiya, Shigeyuki, in Handbook of Advanced Ceramics (Second Edition). 2013, Academic Press: Oxford. p. 429-446.

48. Panda, P.K., Review: environmental friendly lead-free piezoelectric materials. Journal of Materials Science, 2009. 44(19): p. 5049-5062.

49. $\mathrm{Xu}, \mathrm{C}$., et al., Enhanced piezoelectric properties and thermal stability in tetragonal-structured $(\mathrm{Ba}, \mathrm{Ca})(\mathrm{Zr}, \mathrm{Ti}) \mathrm{O}_{3}$ piezoelectrics substituted with trace amount of Mn. Ceramics International, 2016. 42(14): p. 16109-16115.

50. Dunmin, L., et al., Lead-free piezoelectric ceramic $\left(\mathrm{K}_{0.5 \mathrm{Na}} \mathrm{Na}_{0.5}\right) \mathrm{NbO}_{3}$ with $\mathrm{MnO}_{2}$ and $\mathrm{K}_{5.4} \mathrm{Cu}_{1.3} \mathrm{Ta}_{10} \mathrm{O}_{29}$ doping for piezoelectric transformer application. Smart Materials and Structures, 2008. 17(3): p. 035002.

51. Pan, Z., et al., Enhanced piezoelectric properties of tetragonal $\left(\mathrm{Bi}_{1 / 2} \mathrm{~K}_{1 / 2}\right) \mathrm{TiO}_{3}$ lead-free ceramics by substitution of pure Bi-based $\mathrm{Bi}\left(\mathrm{Mg}_{2 / 3} \mathrm{Nb}_{1 / 3}\right) \mathrm{O}_{3}$. Journal of the American Ceramic Society, 2015. 98(1): p. 104-108.

52. Bhatia, D., et al., A novel $\mathrm{ZnO}$ piezoelectric microcantilever energy scavenger: Fabrication and characterization. Sensing and Bio-Sensing Research, 2016. 9: p. 45-52.

53. Korir, K.K., G. Cicero, and A. Catellani, Piezoelectric properties of zinc oxide nanowires: an ab initio study. Nanotechnology, 2013. 24(47): p. 475401.

54. Wang, X., et al., Direct-current nanogenerator driven by ultrasonic waves. Science, 2007. 316(5821): p. 102-5.

55. Dutta, S., A. Antony Jeyaseelan, and S. Sruthi, Ferroelectric and piezoelectric properties of (111) oriented lanthanum modified lead zirconate titanate film. Thin Solid Films, 2014. 562: p. 190-194.

56. Klingshirn, C.F., et al., Zinc Oxide: From Fundamental Properties Towards Novel Applications. 2010: Springer Berlin Heidelberg.

57. Zhou, C. and X. Liu, Dielectric and piezoelectric properties of bismuthcontaining complex perovskite solid solution of 
$\mathrm{Bi}_{1 / 2} \mathrm{Na}_{1 / 2} \mathrm{TiO}_{3}-\mathrm{Bi}\left(\mathrm{Mg}_{2 / 3} \mathrm{Nb}_{1 / 3}\right) \mathrm{O}_{3}$. Journal of Materials Science, 2008. 43(3): p. 1016-1019.

58. Yoo, J., et al., Piezoelectric and dielectric properties of $\mathrm{La}_{2} \mathrm{O}_{3}$ added $\mathrm{Bi}(\mathrm{Na}$, $\mathrm{K}_{\mathrm{TiO}} \mathrm{O}_{3}-\mathrm{SrTiO}_{3}$ ceramics for pressure sensor application. Sensors and Actuators A: Physical, 2006. 126(1): p. 41-47.

59. Li, Y., et al., Dielectric and piezoelecrtic properties of lead-free $\left(\mathrm{Na}_{0.5} \mathrm{Bi}_{0.5}\right) \mathrm{TiO}_{3}-\mathrm{NaNbO}_{3}$ ceramics. Materials Science and Engineering: B, 2004. 112(1): p. 5-9.

60. Yoo, J., et al., Dielectric and piezoelectric characteristics of lead-free Bio.5(Na0.84K $\left.\mathrm{K}_{0.16}\right)_{0.5} \mathrm{TiO}_{3}$ ceramics substituted with Sr. Materials Letters, 2004. 58(29): p. 3831-3835.

61. Ounaies, Z., J.A. Young, and J.S. Harrison, An Overview of the Piezoelectric Phenomenon in Amorphous Polymers, in Field Responsive Polymers. 1999, American Chemical Society. p. 88-103.

62. Martins, P., A.C. Lopes, and S. Lanceros-Mendez, Electroactive phases of poly(vinylidene fluoride): determination, processing and applications. Progress in Polymer Science, 2014. 39(4): p. 683-706.

63. Bauer, S. and F. Bauer, Piezoelectric Polymers and Their Applications, in Piezoelectricity: Evolution and Future of a Technology. 2008, Springer Berlin Heidelberg: Berlin, Heidelberg. p. 157-177.

64. Ribeiro, C., et al., Influence of processing conditions on polymorphism and nanofiber morphology of electroactive poly(vinylidene fluoride) electrospun membranes. Soft Materials, 2010. 8(3): p. 274-287.

65. Mendes, S.F., et al., Effect of filler size and concentration on the structure and properties of poly(vinylidene fluoride)/BaTiO3 nanocomposites. Journal of Materials Science, 2012. 47(3): p. 1378-1388.

66. Patro, T.U., et al., Studies on poly(vinylidene fluoride)-clay nanocomposites: Effect of different clay modifiers. Polymer, 2008. 49(16): p. 3486-3499.

67. Dias, J.C., et al., Electromechanical actuators based on poly(vinylidene fluoride) with [ $\mathrm{N}_{1} 1$ 2016. 51(20): p. 9490-9503.

68. Martins, P., C.M. Costa, and S. Lanceros-Mendez, Nucleation of electroactive $\beta$ phase poly(vinilidene fluoride) with $\mathrm{CoF}_{22} \mathrm{O}_{4}$ and $\mathrm{NiF}_{22} \mathrm{O}_{4}$ nanofillers: a new method for the preparation of multiferroic nanocomposites. Applied Physics A, 2010. 103(1): p. 233-237.

69. $\mathrm{Pu}$, J., et al., Piezoelectric actuation of direct-write electrospun fibers. Sensors and Actuators A: Physical, 2010. 164(1-2): p. 131-136.

70. Whitten, I. and G. Youssef, The effect of ultraviolet radiation on ultrasonic properties of polyurea. Polymer Degradation and Stability, 2016. 123: p. 88-93.

71. Morimoto, M., et al., Structural and electrical characterization of spin-coated polyurea thin films. Polymer, 2015. 79: p. 128-134.

72. Fukada, E. Recent developments of piezoelectric polymers - polyurea, oddnylon, vinylidenecyanide copolymer, and polylactic acid. in 2005 12th International Symposium on Electrets. 2005.

73. Ribeiro, C., et al., Piezoelectric polymers as biomaterials for tissue engineering applications. Colloids Surf B Biointerfaces, 2015. 136: p. 46-55.

74. Khang, G., et al., Preparation and characterization of small intestine submucosa powder impregnated poly(L-lactide) scaffolds: The application for tissue engineered bone and cartilage. Macromolecular Research, 2002. 10(3): p. 158167. 
75. Mark, H.F., Encyclopedia of Polymer Science and Technology, Concise. 2013: Wiley.

76. Park, C., et al., In situ poling and imidization of amorphous piezoelectric polyimides. Polymer, 2004. 45(16): p. 5417-5425.

77. Atkinson, G.M., et al. Novel piezoelectric polyimide MEMS. in TRANSDUCERS, Solid-State Sensors, Actuators and Microsystems, 12th International Conference on, 2003. 2003.

78. Takeshi, H., et al., Piezoelectric and ferroelectric properties of polyurea thin films copolymerized by vapor deposition polymerization of aliphatic and aromatic monomers. Japanese Journal of Applied Physics, 1996. 35(4R): p. 2199.

79. von Berlepsch, H., et al., Piezoelectric activity in a copolymer of acrylonitrile and methylacrylate. IEEE Transactions on Electrical Insulation, 1989. 24(2): p. 357-362.

80. Shigeru, T., T. Takatoshi, and I. Norihiro, Ferro- and pyroelectricity in amorphous polyphenylethernitrile. Japanese Journal of Applied Physics, 1994. 33(10R): p. 5838.

81. Bharti, V., T. Kaura, and R. Nath, Improved piezoelectricity in solvent-cast PVC films. Ieee Transactions on Dielectrics and Electrical Insulation, 1995. 2(6): p. 1106-1110.

82. Briand, D., et al., Micro Energy Harvesting. 2015: Wiley.

83. Fan, F.R., W. Tang, and Z.L. Wang, Flexible nanogenerators for energy harvesting and self-powered electronics. Advanced Materials, 2016. 28(22): p. 4283-4305.

84. Kim, H.S., J.-H. Kim, and J. Kim, A review of piezoelectric energy harvesting based on vibration. International Journal of Precision Engineering and Manufacturing, 2011. 12(6): p. 1129-1141.

85. Wong, V.-K., J.-H. Ho, and A.-B. Chai, Performance of a piezoelectric energy harvester in actual rain. Energy, 2017. 124: p. 364-371.

86. Biswal, A.R., T. Roy, and R.K. Behera, Genetic algorithm- and finite elementbased design and analysis of nonprismatic piezolaminated beam for optimal vibration energy harvesting. Proceedings of the Institution of Mechanical Engineers, Part C: Journal of Mechanical Engineering Science, 2015.

87. Trigona, C., et al. Performance characterization of different nonlineartransduction mechanisms for piezoelectric energy harvesting. in 2014 IEEE International Instrumentation and Measurement Technology Conference (I2MTC) Proceedings. 2014.

88. Ahmadi, M., H.F. Zhang, and J. Tian, Investigation of piezoelectric energy harvesting at elevated temperatures. Ferroelectrics, 2014. 460(1): p. 138-148.

89. Saadon, S. and O. Sidek, Comparative study on standard geometrical structures of cantilever-based MEMS piezoelectric energy harvester over T-shaped cantilever beam for low frequency ambient vibrations. Journal of Optoelectronics and Advanced Materials, 2014. 16(5-6): p. 612-618.

90. Kim, M., et al., Design and fabrication of vibration based energy harvester using microelectromechanical system piezoelectric cantilever for low power applications. Journal of nanoscience and nanotechnology, 2013. 13(12): p. 7932-7937.

91. Kok, S.L., A. Rahman, and M. Fauzi. Design considerations of MEMS based piezoelectric cantilever for harvesting energy. in Applied Electromagnetics (APACE), 2012 IEEE Asia-Pacific Conference on. 2012. 
92. Jambunathan, M., et al. Pulsed laser deposited-PZT based MEMS energy harvesting devices. in Proceedings of ISAF-ECAPD-PFM 2012. 2012.

93. Muralt, P., et al., Vibration energy harvesting with PZT micro device. Procedia Chemistry, 2009. 1(1): p. 1191-1194.

94. Fang, H.-B., et al., Fabrication and performance of MEMS-based piezoelectric power generator for vibration energy harvesting. Microelectronics Journal, 2006. 37(11): p. 1280-1284.

95. Jeon, Y.B., et al., MEMS power generator with transverse mode thin film PZT. Sensors and Actuators A: Physical, 2005. 122(1): p. 16-22.

96. Lu, F., H.P. Lee, and S.P. Lim, Modeling and analysis of micro piezoelectric power generators for micro-electromechanical-systems applications. Smart Materials and Structures, 2004. 13(1): p. 57.

97. Roundy, S., P.K. Wright, and J. Rabaey, A study of low level vibrations as a power source for wireless sensor nodes. Computer Communications, 2003. 26(11): p. 1131-1144.

98. Ewere, F. and G. Wang, Performance of galloping piezoelectric energy harvesters. Journal of Intelligent Material Systems and Structures, 2013.

99. Roundy, S. and P.K. Wright, A piezoelectric vibration based generator for wireless electronics. Smart Materials and Structures, 2004. 13(5): p. 1131.

100. Zhang, G., et al., A low frequency piezoelectric energy harvester with trapezoidal cantilever beam: theory and experiment. Microsystem technologies, 2017. 23(8): p. 3457-3466.

101. Ooi, B.L., J.M. Gilbert, and A.R.A. Aziz, Analytical and finite-element study of optimal strain distribution in various beam shapes for energy harvesting applications. Acta Mechanica Sinica, 2016. 32(4): p. 670-683.

102. Jaafar, M.F.B. and H. Salleh. Performance of piezoelectric energy harvester $(P E H)$ modification using silicon rubber. in The $3^{\text {rd }}$ National Graduate Conference (NatGrad2015), Universiti Tenaga Nasional, Putrajaya Campus. 2015.

103. Chen, Y.-Y., et al., Study of a piezoelectric switching circuit for energy harvesting with bistable broadband technique by work-cycle analysis. Journal of Intelligent Material Systems and Structures, 2012: p. 1-14.

104. Prušáková, L., et al., Modeling and fabrication of single cantilever piezoelectric microgenerator with optimized Zno active layer. Thin Solid Films, 2015. 591: p. 305-310.

105. Papagiannakis, A.T., et al., Development and evaluation of piezoelectric prototypes for roadway energy harvesting. Journal of Energy Engineering, 2017. 143(5): p. 04017034.

106. Song, Y., et al., Road energy harvester designed as a macro-power source using the piezoelectric effect. International Journal of Hydrogen Energy, 2016. 41(29): p. 12563-12568.

107. Hyeoung Woo, K., et al., Energy harvesting using a piezoelectric "cymbal" transducer in dynamic environment. Japanese Journal of Applied Physics, 2004. 43(9R): p. 6178.

108. Kim, H.W., et al., Piezoelectric energy harvesting under high pre-stressed cyclic vibrations. Journal of Electroceramics, 2005. 15(1): p. 27-34.

109. Tufekcioglu, E. and A. Dogan, A flextensional piezo-composite structure for energy harvesting applications. Sensors and Actuators A: Physical, 2014. 216: p. 355-363. 
110. Aktakka, E.E., R.L. Peterson, and K. Najafi. A CMOS-compatible piezoelectric vibration energy scavenger based on the integration of bulk PZT films on silicon. in Electron Devices Meeting (IEDM), 2010 IEEE International. 2010.

111. Aktakka, E.E., R.L. Peterson, and K. Najafi. Thinned-PZT on SOI process and design optimization for piezoelectric inertial energy harvesting. in $201116^{\text {th }}$ International Solid-State Sensors, Actuators and Microsystems Conference. 2011.

112. Soobum, L. and D.Y. Byeng, A design and experimental verification methodology for an energy harvester skin structure. Smart Materials and Structures, 2011. 20(5): p. 057001.

113. Michael, R., et al., Harvesting energy from the motion of human limbs: the design and analysis of an impact-based piezoelectric generator. Smart Materials and Structures, 2009. 18(3): p. 035001.

114. Bayrashev, A., W.P. Robbins, and B. Ziaie, Low frequency wireless powering of microsystems using piezoelectric-magnetostrictive laminate composites. Sensors and Actuators A: Physical, 2004. 114(2-3): p. 244-249.

115. Ericka, M., et al., Energy harvesting from vibration using a piezoelectric membrane. J. Phys. IV France, 2005. 128: p. 187-193.

116. Zou, H.-X., et al., Design and analysis of a piezoelectric vibration energy harvester using rolling mechanism. Journal of Vibration and Acoustics, 2016. 138(5): p. 051007-051007.

117. Kymissis, J., et al. Parasitic power harvesting in shoes. in Wearable Computers, 1998. Digest of Papers. Second International Symposium on. 1998.

118. Platt, S.R., et al., The use of piezoelectric ceramics for electric power generation within orthopedic implants. IEEE/ASME Transactions on Mechatronics, 2005. 10(4): p. 455-461.

119. Duggirala, R., et al. MEMS radioisotope-powered piezoelectric $\mu$-power generator $(R P G)$. in $19^{\text {th }}$ IEEE International Conference on Micro Electro Mechanical Systems. 2006.

120. Wang, Y., et al., Sealed piezoelectric energy harvester driven by hyperbaric air load. Applied Physics Letters, 2016. 108(3): p. 033902.

121. Glynne-Jones, P., S.P. Beeby, and N.M. White, Towards a piezoelectric vibration-powered microgenerator. IEE Proceedings - Science, Measurement and Technology, 2001. 148(2): p. 68-72.

122. Churchill, D.L., et al. Strain energy harvesting for wireless sensor networks. in Proceedings of SPIE - The International Society for Optical Engineering. 2003.

123. Eli, S.L. and K.W. Paul, Resonance tuning of piezoelectric vibration energy scavenging generators using compressive axial preload. Smart Materials and Structures, 2006. 15(5): p. 1413.

124. Koka, A., Z. Zhou, and H.A. Sodano, Vertically aligned $\mathrm{BaTiO}_{3}$ nanowire arrays for energy harvesting. Energy and Environmental Science, 2014. 7(1): p. 288-296.

125. Koka, A. and H.A. Sodano, A low-frequency energy harvester from ultralong, vertically aligned $\mathrm{BaTiO}_{3}$ nanowire arrays. Advanced Energy Materials, 2014. 4(11).

126. Seol, M.-L., et al., Piezoelectric nanogenerator with a nanoforest structure. Nano Energy, 2013. 2(6): p. 1142-1148.

127. Ahmad, M., et al., Enhanced output voltage generation via $\mathrm{ZnO}$ nanowires (50 $n m)$ : Effect of diameter thinning on voltage enhancement. Journal of Physics and Chemistry of Solids, 2017. 104: p. 281-285. 
128. Nour, E.S., et al., Low-frequency self-powered footstep sensor based on $\mathrm{ZnO}$ nanowires on paper substrate. Nanoscale Research Letters, 2016. 11(1): p. 156.

129. Yang, B., et al., Modeling and experimental study of a low-frequency-vibrationbased power generator using $\mathrm{ZnO}$ nanowire arrays. Journal of Microelectromechanical Systems, 2012. 21(4): p. 776-778.

130. Nour, E.S., et al., Low frequency accelerator sensor based on piezoelectric ZnO nanorods grown by low temperature scalable process. physica status solidi (a), 2016. 213(9): p. 2503-2508.

131. Jeong, C.K., et al., Comprehensive biocompatibility of nontoxic and high-output flexible energy harvester using lead-free piezoceramic thin film. APL Materials, 2017. 5(7): p. 074102.

132. Bejarano, F., A. Feeney, and M. Lucas, A cymbal transducer for power ultrasonics applications. Sensors and Actuators A: Physical, 2014. 210: p. 182189.

133. Shen, D., et al., Micromachined PZT cantilever based on SOI structure for low frequency vibration energy harvesting. Sensors and Actuators A: Physical, 2009. 154(1): p. 103-108.

134. Nour, E.S., et al., Handwriting enabled harvested piezoelectric power using ZnO nanowires/polymer composite on paper substrate. Nano Energy, 2014. 9: p. 221-228.

135. Li, Y., Z. Çelik-Butler, and D.P. Butler, An integrated piezoelectric zinc oxide nanowire micro-energy harvester. Nano Energy, 2016. 26: p. 456-465.

136. Vijaya, M.S., Piezoelectric Materials and Devices: Applications in Engineering and Medical Sciences. 2016: CRC Press.

137. Alomari, A., et al., A multisource energy harvesting utilizing highly efficient ferroelectric PMN-PT single crystal. Journal of Materials Science: Materials in Electronics, 2016. 27(10): p. 10020-10030.

138. Yang, Z. and J. Zu, Comparison of PZN-PT, PMN-PT single crystals and PZT ceramic for vibration energy harvesting. Energy Conversion and Management, 2016. 122: p. 321-329.

139. Erturk, A., O. Bilgen, and D.J. Inman, Power generation and shunt damping performance of a single crystal lead magnesium niobate-lead zirconate titanate unimorph: Analysis and experiment. Applied Physics Letters, 2008. 93(22): p. 224102.

140. Dow, A.B.A., et al., Design, fabrication and testing of a piezoelectric energy microgenerator. Microsystem technologies, 2014. 20(4-5): p. 1035-1040.

141. Alluri, N.R., et al., Piezoelectric BaTiO3/alginate spherical composite beads for energy harvesting and self-powered wearable flexion sensor. Composites Science and Technology, 2017. 142: p. 65-78.

142. Wu, F., et al., Energy scavenging based on a single-crystal PMN-PT nanobelt. Scientific reports, 2016. 6.

143. Patel, S., et al., Cyclic piezoelectric energy harvesting in PMN-PT single crystals. Ferroelectrics, 2015. 481(1): p. 138-145.

144. Hwang, G.-T., et al., Self-powered cardiac pacemaker enabled by flexible single crystalline PMN-PT piezoelectric energy harvester. Advanced Materials, 2014. 26(28): p. 4880-4887.

145. $\mathrm{Xu}, \mathrm{C}$., et al., Cantilever driving low frequency piezoelectric energy harvester using single crystal material $0.71 \mathrm{~Pb}\left(\mathrm{Mg}_{1 / 3} \mathrm{Nb}_{2 / 3}\right) \mathrm{O}_{3}-0.29 \mathrm{PbTiO}_{3}$. Applied Physics Letters, 2012. 101(3): p. 033502. 
146. Boulbar, E.D.L. and C.R. Bowen. Study of $\mathrm{Y}$-cut $\mathrm{LiNbO}_{3}(010)$ crystal under oscillated vibration at high temperature for energy harvesting in hostile environment. in 2015 Joint IEEE International Symposium on the Applications of Ferroelectric (ISAF), International Symposium on Integrated Functionalities (ISIF), and Piezoelectric Force Microscopy Workshop (PFM). 2015.

147. Dong, W.D., J.A. Gallagher, and C.S. Lynch, Ideal energy harvesting cycle using a phase transformation in ferroelectric crystals. Smart Materials and Structures, 2014. 23(12).

148. Dong, W., et al. Mechanical energy harvesting utilizing phase transition in 32 mode relaxor-ferroelectric PIN-PMN-PT single crystals. in Proceedings of SPIE - The International Society for Optical Engineering. 2012.

149. Wu, M., et al., Multi-resonant wideband energy harvester based on a folded asymmetric M-shaped cantilever. AIP Advances, 2015. 5(7): p. 077149.

150. Liu, W., et al., Low frequency wide bandwidth MEMS energy harvester based on spiral-shaped PVDF cantilever. Science China Technological Sciences, 2014. 57(6): p. 1068-1072.

151. Li, S., J. Yuan, and H. Lipson, Ambient wind energy harvesting using cross-flow fluttering. Journal of Applied Physics, 2011. 109(2): p. 026104.

152. Li, B., et al., Harvesting low-frequency acoustic energy using quarterwavelength straight-tube acoustic resonator. Applied Acoustics, 2013. 74(11): p. 1271-1278.

153. Mhetre, M.R. and H.K. Abhyankar, Human exhaled air energy harvesting with specific reference to PVDF film. Engineering Science and Technology, an International Journal, 2017. 20(1): p. 332-339.

154. Jung, I., et al., Flexible piezoelectric polymer-based energy harvesting system for roadway applications. Applied Energy, 2017. 197: p. 222-229.

155. Orrego, S., et al., Harvesting ambient wind energy with an inverted piezoelectric flag. Applied Energy, 2017. 194: p. 212-222.

156. Fengchi, L., et al. Simulation study for energy harvesting using polyvinylidene fluoride flag in the flow. in $3^{\text {rd }}$ International Conference on Material, Mechanical and Manufacturing Engineering (IC3ME 2015). 2015.

157. Bischur, E. and N. Schwesinger. Piezoelectric PVDF film energy harvester for powering a wireless sensor system. in Proc. SPIE 8688, Active and Passive Smart Structures and Integrated Systems 2013. 2013. International Society for Optics and Photonics.

158. Fuh, Y.K., et al., Self-powered active sensor with concentric topography of piezoelectric fibers. Nanoscale Research Letters, 2017. 12(1): p. 44.

159. Mokhtari, F., et al., Comparative evaluation of piezoelectric response of electrospun PVDF (polyvinilydine fluoride) nanofiber with various additives for energy scavenging application. The Journal of The Textile Institute, 2016: p. 19.

160. Nunes-Pereira, J., et al., Energy harvesting performance of piezoelectric electrospun polymer fibers and polymer/ceramic composites. Sensors and Actuators A: Physical, 2013. 196: p. 55-62.

161. Zhu, J., L. Jia, and R. Huang, Electrospinning poly(l-lactic acid) piezoelectric ordered porous nanofibers for strain sensing and energy harvesting. Journal of Materials Science: Materials in Electronics, 2017. 28(16): p. 12080-12085.

162. Nunes-Pereira, J., et al., Energy harvesting performance of $\mathrm{BaTiO}_{3} /$ poly(vinylidene fluoride-trifluoroethylene) spin coated nanocomposites. Composites Part B: Engineering, 2015. 72: p. 130-136. 
163. Chen, X., et al. Nanowire-based flexible P(VDF-TrFE) nanogenerator for simultaneously harvesting mechanical and thermal energies. in Nanotechnology (IEEE-NANO) , 2015 IEEE 15 ${ }^{\text {th }}$ International Conference on. 2015.

164. Sunghyun, Y. and C. Young-Ho, A skin-attachable flexible piezoelectric pulse wave energy harvester. Journal of Physics: Conference Series, 2014. 557(1): p. 012026.

165. Wang, F., et al., High quality barium titanate nanofibers for flexible piezoelectric device applications. Sensors and Actuators A: Physical, 2015. 233: p. 195-201.

166. Lee, C., et al., Electrospun uniaxially-aligned composite nanofibers as highlyefficient piezoelectric material. Ceramics International, 2016. 42(2, Part A): p. 2734-2740.

167. Park, J.-I., et al., Flexible ceramic-elastomer composite piezoelectric energy harvester fabricated by additive manufacturing. Journal of Composite Materials, 2015: p. 1-7.

168. Seveno, R., et al., Flexible PET/Al/PZT/Al/PET multi-layered composite for low frequency energy harvesting. Journal of Physics D: Applied Physics, 2017. 50(16).

169. Abinnas, N., et al., 0.8 V nanogenerator for mechanical energy harvesting using bismuth titanate-PDMS nanocomposite. Applied Surface Science, 2017. 418: p. 362-368.

170. Yaqoob, U., A.S.M.I. Uddin, and G.-S. Chung, A novel tri-layer flexible piezoelectric nanogenerator based on surface-modified graphene and PVDF$\mathrm{BaTiO}_{3}$ nanocomposites. Applied Surface Science, 2017. 405: p. 420-426.

171. Schädli, G.N., R. Büchel, and S.E. Pratsinis, Nanogenerator power output: influence of particle size and crystallinity of $\mathrm{BaTiO}_{3}$. Nanotechnology, 2017. 28(27).

172. Sung-Ho, S., et al., Solvent-assisted optimal BaTiO 3 nanoparticles-polymer composite cluster formation for high performance piezoelectric nanogenerators. Nanotechnology, 2014. 25(48): p. 485401.

173. Ou, C., et al., Template-assisted hydrothermal growth of aligned zinc oxide nanowires for piezoelectric energy harvesting applications. ACS Applied Materials \& Interfaces, 2016. 8(22): p. 13678-13683.

174. $\mathrm{Xu}, \mathrm{S}$., et al., Flexible piezoelectric PMN-PT nanowire-based nanocomposite and device. Nano Letters, 2013. 13(6): p. 2393-2398.

175. Yu, Y., et al., Biocompatibility and in vivo operation of implantable mesoporous PVDF-based nanogenerators. Nano Energy, 2016. 27: p. 275-281.

176. Kumar Biswal, A., S. Das, and A. Roy. Designing and synthesis of a polymer matrix piezoelectric composite for energy harvesting. in IOP Conference Series: Materials Science and Engineering. 2017.

177. Zeng, Z., et al., A plastic-composite-plastic structure high performance flexible energy harvester based on PIN-PMN-PT single crystal/epoxy 2-2 composite. Applied Physics Letters, 2017. 110(10).

178. Alluri, N.R., et al., Scavenging biomechanical energy using high-performance, flexible $\mathrm{BaTiO}_{3}$ nanocube/PDMS composite films. ACS Sustainable Chemistry and Engineering, 2017. 5(6): p. 4730-4738.

179. Guo, R., et al., Synthesis of orthorhombic perovskite-type $\mathrm{ZnSnO}_{3}$ single-crystal nanoplates and their application in energy harvesting. ACS Applied Materials and Interfaces, 2017. 9(9): p. 8271-8279. 
180. Adhikary, P. and D. Mandal, Enhanced electro-active phase in a luminescent $P(V D F-H F P) / Z n^{2+}$ flexible composite film for piezoelectric based energy harvesting applications and self-powered UV light detection. Physical Chemistry Chemical Physics, 2017. 19(27): p. 17789-17798.

181. Bhunia, R., et al., Flexible nano-ZnO/polyvinylidene difluoride piezoelectric composite films as energy harvester. Applied Physics A, 2016. 122(7): p. 1-13.

182. Jeong, C.K., et al., A hyper-stretchable elastic-composite energy harvester. Advanced Materials, 2015. 27(18): p. 2866-2875.

183. Tang, G., et al., Development of high performance piezoelectric $d_{33}$ mode MEMS vibration energy harvester based on PMN-PT single crystal thick film. Sensors and Actuators A: Physical, 2014. 205: p. 150-155.

184. Dhakar, L., et al., A new energy harvester design for high power output at low frequencies. Sensors and Actuators A: Physical, 2013. 199: p. 344-352.

185. Bowland, C.C., M.H. Malakooti, and H.A. Sodano, Barium titanate film interfaces for hybrid composite energy harvesters. ACS Applied Materials and Interfaces, 2017. 9(4): p. 4057-4065.

186. Chen, X., et al., High-performance piezoelectric nanogenerators with Imprinted $P(V D F-T r F E) / B \mathrm{CTiO}_{3}$ nanocomposite micropillars for self-powered flexible sensors. Small, 2017. 13(23).

187. Jeong, C.K., et al., Virus-directed design of a flexible $\mathrm{BaTiO}_{3}$ nanogenerator. ACS Nano, 2013. 7(12): p. 11016-11025.

188. Ding, R., et al., High-performance piezoelectric nanogenerators composed of formamidinium lead halide perovskite nanoparticles and poly(vinylidene fluoride). Nano Energy, 2017. 37: p. 126-135. 


\section{List of symbols and acronyms}

\begin{tabular}{|c|c|}
\hline ADP & Ammonium dihydrogen phosphate \\
\hline BiT & Bismuth titanate \\
\hline BKT & Bismuth potassium titanate \\
\hline BNT & Bismuth sodium titanate \\
\hline Ca-alg & Calcium alginate polymer \\
\hline$D$ & Electric displacement vector \\
\hline$d$ & Piezoelectric strain coefficient \\
\hline DKT & Dipotassium tartrate \\
\hline$E$ & Electric field vector \\
\hline EDT & Ethylene diamine tartrate \\
\hline$f$ & Frequency \\
\hline $\mathrm{FAPbBr}_{3}$ & Formamidinium lead halide perovskite \\
\hline FDA & Food and Drug Administration \\
\hline$g$ & Gravitational acceleration \\
\hline HFP & Hexafluoropropylene \\
\hline ITO & Indium tin oxide \\
\hline$k$ & Electromechanical coupling factor \\
\hline KNN & Potassium-sodium niobates \\
\hline KNN-KCT-Mn & KNN-potassium cooper titanate- manganese \\
\hline MEMS & Microelectromechanical systems \\
\hline MPB & Morphotropic Phase Boundary \\
\hline MWCNT & Multi-walled carbon nanotubes \\
\hline$n$-Gr & $n$-type graphene \\
\hline$p$ & Electric dipole moment \\
\hline$P$ & Polarization \\
\hline $\mathrm{P}(\mathrm{VDF}-\mathrm{HFP})$ & Poly(vinylidene fluoride-co-hexafluoropropylene) \\
\hline $\mathrm{P}(\mathrm{VDF}-\mathrm{TrFE})$ & Poly(vinylidenefluoride-co-trifluoroethylene) \\
\hline PAN & Polyacrylonitrile \\
\hline $\mathrm{PC}$ & Polycarbonate \\
\hline PDMS & Polydimethylsiloxane \\
\hline PEDOT:PSS & Poly(3,4-ethylenedioxythiophene) polystyrene sulfonate \\
\hline PET & Polyethylene terephthalate \\
\hline PGA & Polyglycolide \\
\hline PIN-PMN-PT & Lead indium niobate- PMN-PT \\
\hline PLA & Polylactide \\
\hline PLGA & Poly(lactide-coglycolide) \\
\hline PLLA & Poly(L-latic acid) \\
\hline PMN & Lead magnesium niobate \\
\hline PMN-PT & Lead magnesium niobate - lead titanate \\
\hline PPEN & Poly(phenyl ether nitrile) \\
\hline PVAc & Polyvinyl acetate \\
\hline PVC & Polyvinyl chloride \\
\hline PVDC & Poly(vinylidene chloride) \\
\hline PVDCN/VAc & Poly(vinylidene cyanide-vinyl acetate) \\
\hline PVDF & Poly(vinylidene fluoride) \\
\hline PZN & Lead zinc niobate \\
\hline PZN-PT & Lead zinc niobate-lead titanate \\
\hline PZT & Lead zirconate titanate \\
\hline$Q$ & Mechanical quality factor \\
\hline$q$ & Electric charge \\
\hline$S$ & Strain tensors \\
\hline
\end{tabular}




\begin{tabular}{|l|l|}
\hline$S$ & Elastic compliance matrix \\
\hline$T$ & Stress tensors \\
\hline$T_{c}$ & Curie temperature \\
\hline$T_{d}$ & Depolarization temperature \\
\hline$T_{g}$ & Glass transition temperature \\
\hline$x$ & Distance \\
\hline$\alpha$ & Alpha \\
\hline$\beta$ & Beta \\
\hline$\gamma$ & Gamma \\
\hline$\varepsilon$ & Dielectric permittivity \\
\hline$\varepsilon_{0}$ & Permittivity of free space \\
\hline$\chi$ & Electric susceptibility \\
\hline
\end{tabular}

